\title{
ON CARLEMAN ESTIMATES FOR ELLIPTIC AND PARABOLIC OPERATORS. APPLICATIONS TO UNIQUE CONTINUATION AND CONTROL OF PARABOLIC EQUATIONS *,**
}

\author{
JÉrôme Le Rousseau ${ }^{1}$ And Gilles LeBeau ${ }^{2}$
}

\begin{abstract}
Local and global Carleman estimates play a central role in the study of some partial differential equations regarding questions such as unique continuation and controllability. We survey and prove such estimates in the case of elliptic and parabolic operators by means of semi-classical microlocal techniques. Optimality results for these estimates and some of their consequences are presented. We point out the connexion of these optimality results to the local phase-space geometry after conjugation with the weight function. Firstly, we introduce local Carleman estimates for elliptic operators and deduce unique continuation properties as well as interpolation inequalities. These latter inequalities yield a remarkable spectral inequality and the null controllability of the heat equation. Secondly, we prove Carleman estimates for parabolic operators. We state them locally in space at first, and patch them together to obtain a global estimate. This second approach also yields the null controllability of the heat equation.
\end{abstract}

Mathematics Subject Classification. 35B60, 35J15, 35K05, 93B05, 93B07.

Received July 13, 2009. Revised January 25, 2011.

Published online October 21, 2011.

\section{INTRODUCTION}

In 1939, T. Carleman introduced some energy estimates with exponential weights to prove a uniqueness result for some elliptic partial differential equations (PDE) with smooth coefficients in dimension two [11]. This type of estimate, now referred to as Carleman estimates, were generalized and systematized by Hörmander and others for a large class of differential operators in arbitrary dimensions (see [25], Chap. 8 and [26], Sects. 28.1-2; see also [59]).

In more recent years, the field of applications of Carleman estimates has gone beyond the original domain they had been introduced for, i.e., a quantitative result for unique continuation. They are also used in the study

Keywords and phrases. Carleman estimates, semiclassical analysis, elliptic operators, parabolic operators, controllability, observability.

* The CNRS Pticrem project facilitated the writting of these notes. The first author was partially supported by l'Agence Nationale de la Recherche under grant ANR-07-JCJC-0139-01.

** M. Bellassoued's handwritten notes of [38] were very valuable to us and we wish to thank him for letting us use them. The authors wish to thank L. Robbiano for many discussions on the subject of these notes and L. Miller for discussions on some of the optimality results. We also thank M. Léautaud for his corrections.

${ }^{1}$ Université d'Orléans, Laboratoire Mathématiques et Applications, Physique Mathématique d'Orléans, CNRS UMR 6628, Fédération Denis Poisson, FR CNRS 2964, B.P. 6759, 45067 Orléans Cedex 2, France. jlr@univ-orleans.fr

2 Université de Nice Sophia-Antipolis, Laboratoire Jean Dieudonné, UMR CNRS 6621, Parc Valrose, 06108 Nice Cedex 02, France. gilles.lebeau@unice.fr 
of inverse problems and control theory for PDEs. Here, we shall mainly survey the application to control theory in the case of parabolic equations, for which Carleman estimates have now become an essential technique.

In control of PDEs, for evolution equations, one aims to drive the solution in a prescribed state, starting from a certain initial condition. One acts on the equation through a source term, a so-called distributed control, or through a boundary condition, a so-called boundary control. To achieve general results one wishes for the control to only act in part of the domain or its boundary and one wishes to have as much latitude as possible in the choice of the control region: location, size.

As already mentioned, we focus our interest on the heat equation here. In a smooth and bounded ${ }^{1}$ domain $\Omega$ in $\mathbb{R}^{n}$, for a time interval $(0, T)$ with $T>0$, and for a distributed control we consider

$$
\begin{cases}\partial_{t} y-\Delta y=1_{\omega} v & \text { in } Q=(0, T) \times \Omega, \\ y=0 & \text { on } \Sigma=(0, T) \times \partial \Omega, \\ y(0)=y_{0} & \text { in } \Omega .\end{cases}
$$

Here $\omega \Subset \Omega$ is an interior control region. The null controllability of this equation, that is the existence, for any $y_{0} \in L^{2}(\Omega)$, of a control $v \in L^{2}(Q)$, with $\|v\|_{L^{2}(Q)} \leq C\left\|y_{0}\right\|_{L^{2}(Q)}$, such that $y(T)=0$, was first proven in [39], by means of Carleman estimates for the elliptic operator $-\partial_{s}^{2}-\Delta_{x}$ in a domain $Z=\left(0, S_{0}\right) \times \Omega$ with $S_{0}>0$. A second approach, introduced in [22], also led to the null controllability of the heat equation. It is based on global Carleman estimates for the parabolic operator $\partial_{t}-\Delta$. These estimates are said to be global for they apply to functions that are defined in the whole domain $(0, T) \times \Omega$ and that solely satisfy boundary condition, e.g., homogeneous Dirichlet boundary conditions on $(0, T) \times \partial \Omega$.

We shall first survey the approach of [39], proving and using local elliptic Carleman estimates. We prove such estimates with techniques from semi-classical microlocal analysis. The estimates we prove are local in the sense that they apply to functions whose support is localized in a closed region strictly included in $\bar{\Omega}$. With these estimates at hand, we derive interpolation inequalities for functions in $Z=\left(0, S_{0}\right) \times \Omega$, that satisfy some boundary conditions, and we derive a spectral inequality for finite linear combinations of eigenfunctions of the Laplace operator in $\Omega$ with homogeneous Dirichlet boundary conditions. This yields an iterative construction of the control function $v$ working in increasingly larger finite dimensional subspaces.

The method introduced in [39] was further extended to address thermoelasticity [41], thermoelastic plates [6], semigroups generated by fractional orders of elliptic operators [45]. It has also been used to prove null controllability results in the case of non smooth coefficients $[8,35]$. Local Carleman estimates have also been central in the study of other types of PDEs for instance to prove unique continuation results $[21,48,51,53,54]$ and to prove stabilization results $[5,40]$ to cite a few. Here, we shall consider self-adjoint elliptic operators, in particular the Laplace operator. The method of [39] can also be extended to some non selfadjoint case, e.g. non symmetric systems [37].

In a second part we survey the approach of [22], that is by means of global parabolic Carleman estimates. These estimates are characterized by an observation term. Such an estimate readily yields a so-called observability inequality for the parabolic operator, which is equivalent to the null controllability of the linear system (1.1). The proof of parabolic Carleman estimates we provide is new and different from that given in [22]. In [22] the estimate is derived through numerous integrations by parts and the identification of positive "dominant" terms. As in the elliptic case of the first part, we base our analysis on semi-classical microlocal analysis. In particular, the estimate is obtained through a time-uniform semi-classical Gårding inequality. In the case of parabolic operators, we first prove local estimates and we also show how such estimates can be patched together to finally yield a global estimate with an observation term in the form of that proved by [22].

The approach of [22] has been successful, allowing one to also treat the controllability of more general parabolic equations. Time dependent terms can be introduced in the parabolic equation. Moreover, one may consider the controllability of some semi-linear parabolic equations. For these questions we refer to $[4,15,18,22]$. In fact, global Carleman estimates yield a precise knowledge of the "cost" of the control function in the linear

\footnotetext{
${ }^{1}$ The problem of null-controllability of the heat equation in the case where $\Omega$ is unbounded is entirely different $[43,44,46]$.
} 
case [17] which allows to carry out a fix point argument after linearization of the semi-linear equation. The results on semi-linear equations have been extended to the case of non smooth coefficients $[7,14,34,36]$. The use of global parabolic Carleman estimates has also allowed to address the controllability of non linear equations such as the Navier-Stokes equations [19,29], the Boussinesq system [20], fluid structure systems [9,30], weakly coupled parabolic systems $[12,23,32,33]$ to cite a few. A review of the application of global parabolic Carleman estimates can be found in [16].

A local Carleman estimates takes the following form. For an elliptic operator $P$ and for a well-chosen weight function $\varphi=\varphi(x)$, there exists $C>0$ and $h_{1}>0$ such that

$$
h\left\|\mathrm{e}^{\varphi / h} u\right\|_{0}^{2}+h^{3}\left\|\mathrm{e}^{\varphi / h} \nabla_{x} u\right\|_{0}^{2} \leq C h^{4}\left\|\mathrm{e}^{\varphi / h} P u\right\|_{0}^{2},
$$

for $u$ smooth with compact support and $0<h \leq h_{1}$.

In this type of estimate we can take the parameter $h$ as small as needed, which is often done in applications to inverse problems or control theory. For this reason, it appeared sensible to us to present results regarding the optimality of the powers of the parameter $h$ in such Carleman estimates. For example, in the case of parabolic estimates this question is crucial for the application to the controllability of semilinear parabolic equations (see e.g. $[14,18])$. To make precise such optimality result we present its connection to the local phase-space geometry. We show that the presences of $h$ in front of the first term and $h^{3}$ in front of the second term in (1.2) are connected to the characteristic set of the conjugated operator $P_{\varphi}=h^{2} \mathrm{e}^{\varphi / h} P \mathrm{e}^{-\varphi / h}$. Away from this characteristic set, a better estimate can be achieved.

If $\omega$ is an open subset of $\Omega$, from elliptic Carleman estimates we obtain a spectral inequality of the form

$$
\|u\|_{L^{2}(\Omega)}^{2} \leq C \mathrm{e}^{C \sqrt{\mu}}\|u\|_{L^{2}(\omega)}^{2}
$$

for some $C>0$ and for $u$ a linear combination of eigenfunctions of $-\Delta$ associated to eigenvalues less than $\mu>0$. An optimality result for such an inequality is also presented as well as some unique continuation property for series of eigenfunctions of $-\Delta$. This spectral inequality is also at the center of the construction of the control function of (1.1) and the estimation of its "cost" for particular frequency ranges.

An important point in the derivation of a Carleman estimate consist in the choice of the weight function $\varphi$. A necessary condition can be derived. This condition concerns the sub-ellipticity of the symbol of the conjugated operator $P_{\varphi}$. With the approach we use, the sufficiency of this condition is obtained. We also consider stronger sufficient conditions: the method introduced by [22] to derive Carleman estimates is analyzed in this framework.

This article originates in part from a lecture given by Lebeau at the Faculté des Sciences in Tunis in February 2005 and from Bellassoued's handwritten notes taken on this occasion [38].

\subsection{Outline}

We start by briefly introducing semi-classical pseudodifferential operators ( $\psi$ DO) in Section 2. The Gårding inequality will be one of the important tools we introduce. It will allow us to quickly derive a local Carleman estimate for an elliptic operator in Section 3. In that section, we present the sub-ellipticity condition that the weight function has to fulfill. We also show the optimality of the powers of the semi-classical parameter $h$ in the Carleman estimates. We apply Carleman estimates to elliptic equations and inequalities and prove unique continuation results in Section 4. In Section 5, we prove the interpolation and spectral inequalities. The latter inequality concerns finite linear combinations of eigenfunctions of the elliptic operator. We show the optimality of the constant $\mathrm{e}^{C \sqrt{\mu}}$ in this inequality where $\mu$ is the largest eigenvalue considered in the sum. We also prove a unique continuation property for some series of such eigenfunctions. In Section 6, these results are applied to construct a control function for the parabolic equation (1.1). Section 7 is devoted to parabolic Carleman estimates. We first prove them locally in space with a uniform-in-time Gårding inequality. We then patch them together to obtain a global estimate. We provide a second proof of the null controllability of parabolic equations with this approach.

For a clearer exposition, some of the results given in the main sections are proven in the appendices. 


\subsection{Notation}

The notation we use is classical. The canonical inner product on $\mathbb{R}^{n}$ is denoted by $\langle.,$.$\rangle , the associated$ Euclidean norm by $|$.$| and the Euclidean open ball with center x$ and radius $r$ by $B(x, r)$. For $\xi \in \mathbb{R}^{n}$ we set $\langle\xi\rangle:=\left(1+|\xi|^{2}\right)^{\frac{1}{2}}$. If $\alpha$ is a multi-index, i.e., $\alpha=\left(\alpha_{1}, \ldots, \alpha_{n}\right) \in \mathbb{N}^{n}$, we introduce

$$
\xi^{\alpha}=\xi_{1}^{\alpha_{1}} \cdots \xi_{n}^{\alpha_{n}}, \quad \text { if } \xi \in \mathbb{R}^{n}, \quad \partial^{\alpha}=\partial_{x_{1}}^{\alpha_{1}} \cdots \partial_{x_{n}}^{\alpha_{n}}, \quad D^{\alpha}=D_{x_{1}}^{\alpha_{1}} \cdots D_{x_{n}}^{\alpha_{n}}, \quad \text { and } \quad|\alpha|=\alpha_{1}+\cdots+\alpha_{n}
$$

where $D=\frac{h}{i} \partial$. In $\mathbb{R}^{n}$, we denote by $\nabla$ the gradient $\left(\partial_{x_{1}}, \ldots, \partial_{x_{n}}\right)^{t}$ and by $\Delta$ the Laplace operator $\partial_{x_{1}}^{2}+\cdots+\partial_{x_{n}}^{2}$. If needed the variables along which differentiations are performed will be made clear by writing $\nabla_{x}$ or $\Delta_{x}$ for instance. We shall also write $\varphi^{\prime}=\nabla_{x} \varphi$.

For an open set $\Omega$ in $\mathbb{R}^{n}$, we denote by $\mathscr{C}_{c}^{\infty}(\Omega)$ the set of functions of class $\mathscr{C}^{\infty}$ whose support is a compact subset of $\Omega$. For a compact set $K$ in $\mathbb{R}^{n}$, we denote by $\mathscr{C}_{c}^{\infty}(K)$ the set of functions in $\mathscr{C}_{c}^{\infty}\left(\mathbb{R}^{n}\right)$ whose support is in $K$. The Schwartz space $\mathscr{S}\left(\mathbb{R}^{n}\right)$ is the set of functions of class $\mathscr{C}^{\infty}$ that decrease rapidly at infinity. Its dual, $\mathscr{S}^{\prime}\left(\mathbb{R}^{n}\right)$, is the set of tempered distributions. The Fourier transform of a function $u \in \mathscr{S}\left(\mathbb{R}^{n}\right)$ is defined by $\hat{u}(\xi)=\int \mathrm{e}^{-\mathrm{i}\langle x, \xi\rangle} u(x) \mathrm{d} x$, with an extension by duality to $\mathscr{S}^{\prime}\left(\mathbb{R}^{n}\right)$.

Let $\Omega$ be an open subset of $\mathbb{R}^{n}$. The space $L^{2}(\Omega)$ of square integrable functions is equipped with the hermitian inner product $(u, v)_{L^{2}}=\int_{\Omega} u(x) \bar{v}(x) \mathrm{d} x$ and the associated norm $\|u\|_{L^{2}}=\|u\|_{0}=(u, u)_{L^{2}}^{1 / 2}$. In $\mathbb{R}^{n}$, classical Sobolev spaces are defined by $H^{s}\left(\mathbb{R}^{n}\right)=\left\{u \in \mathscr{S}^{\prime}\left(\mathbb{R}^{n}\right) ;\langle\xi\rangle^{s} \hat{u} \in L^{2}\left(\mathbb{R}^{n}\right)\right\}$ for all $s \in \mathbb{R}$. In $\Omega$, for $s \in \mathbb{N}, H^{s}(\Omega)$ is defined by $H^{s}(\Omega)=\left\{u \in \mathscr{D}^{\prime}(\Omega) ; \partial^{\alpha} u \in L^{2}\left(\mathbb{R}^{n}\right), \forall \alpha \in \mathbb{N}^{n},|\alpha| \leq s\right\}$.

For two functions $f$ and $g$ with variables $x, \xi$ in $\mathbb{R}^{n} \times \mathbb{R}^{n}$, we defined their so-called Poisson bracket

$$
\{f, g\}=\sum_{j}\left(\partial_{\xi_{j}} f \partial_{x_{j}} g-\partial_{x_{j}} f \partial_{\xi_{j}} g\right)
$$

For two operators $A, B$ their commutator will be denoted $[A, B]=A B-B A$.

In these notes, $C$ will always denote a generic positive constant whose value can be different in each line. If we want to keep track of the value of a constant we shall use other letters. We shall sometimes write $C_{\lambda}$ for a generic constant that depends on a parameter $\lambda$.

\section{Preliminaries: Semi-Classical (PSeudo-)Differential operators}

Semi-classical theory originates from quantum physics. The scaling parameter $h$ we introduce is consistent with Plank's constant in physics. It will be assumed small: $h \in\left(0, h_{0}\right), 0<h_{0}<<1$. We set $D=\frac{h}{i} \partial$. The semi-classical limit corresponds to $h \rightarrow 0$.

If $p(x, \xi)$ is a polynomial in $\xi$ of order less than or equal to $m, x, \xi \in \mathbb{R}^{n}, p(x, \xi)=\sum_{|\alpha| \leq m} a_{\alpha}(x) \xi^{\alpha}$, we set

$$
p(x, D) u=\sum_{|\alpha| \leq m} a_{\alpha}(x) D^{\alpha} u
$$

Here, $\alpha$ is a multi-index. We observe that $D^{\alpha} u=\frac{h^{|\alpha|}}{(2 \pi)^{n}} \int_{\mathbb{R}^{n}} \mathrm{e}^{\mathrm{i}\langle x, \xi\rangle} \xi^{\alpha} \hat{u}(\xi) \mathrm{d} \xi$, for $u \in \mathscr{S}\left(\mathbb{R}^{n}\right)$, where $\hat{u}$ is the classical Fourier transform of $u$. We thus have

$$
p(x, D) u(x)=\sum_{|\alpha| \leq m} \frac{h^{|\alpha|}}{(2 \pi)^{n}} \int \mathrm{e}^{\mathrm{i}\langle x, \xi\rangle} a_{\alpha}(x) \xi^{\alpha} \hat{u}(\xi) \mathrm{d} \xi=(2 \pi h)^{-n} \int \mathrm{e}^{\mathrm{i}\langle x, \xi\rangle / h} \sum_{|\alpha| \leq m} a_{\alpha}(x) \xi^{\alpha} \hat{u}(\xi / h) \mathrm{d} \xi
$$

or, formally, $p(x, D) u(x)=(2 \pi h)^{-n} \iint \mathrm{e}^{\mathrm{i}\langle x-y, \xi\rangle / h} p(x, \xi) u(y) \mathrm{d} y \mathrm{~d} \xi$. More generally we introduce the following symbol classes. 
Definition 2.1. Let $a(x, \xi, h) \in \mathscr{C}^{\infty}\left(\mathbb{R}^{n} \times \mathbb{R}^{n}\right)$, with $h$ as a parameter in $\left(0, h_{0}\right)$, be such that for all multi-indices $\alpha, \beta$ we have

$$
\left|\partial_{x}^{\alpha} \partial_{\xi}^{\beta} a(x, \xi, h)\right| \leq C_{\alpha, \beta}\langle\xi\rangle^{m-|\beta|}, \quad x \in \mathbb{R}^{n}, \xi \in \mathbb{R}^{n}, h \in\left(0, h_{0}\right) .
$$

We write $a \in S^{m}$.

For $a \in S^{m}$ we call principal symbol the equivalence class of $a$ in $S^{m} /\left(h S^{m-1}\right)$.

Lemma 2.2. Let $m \in \mathbb{R}$ and $a_{j} \in S^{m-j}$ with $j \in \mathbb{N}$. Then there exists $a \in S^{m}$ such that

$$
\forall N \in \mathbb{N}, \quad a-\sum_{j=0}^{N} h^{j} a_{j} \in h^{N+1} S^{m-N-1} .
$$

We then write $a \sim \sum_{j} h^{j} a_{j}$. The symbol a is unique up to $\mathcal{O}\left(h^{\infty}\right) S^{-\infty}$, in the sense that the difference of two such symbols is in $\mathcal{O}\left(h^{N}\right) S^{-M}$ for all $N, M \in \mathbb{N}$.

We identify $a_{0}$ with the principal symbol of $a$. In general, for the symbols of the form $a \sim \sum_{j} h^{j} a_{j}$ that we shall consider here the symbols $a_{j}$ will not depend on the scaling parameter $h$.

With these symbol classes we can define pseudodifferential operators ( $\psi$ DOs).

Definition 2.3. If $a \in S^{m}$, we set

$$
\begin{aligned}
a(x, D, h) u(x)=\operatorname{Op}(a) u(x): & =(2 \pi h)^{-n} \iint \mathrm{e}^{\mathrm{i}\langle x-y, \xi\rangle / h} a(x, \xi, h) u(y) \mathrm{d} y \mathrm{~d} \xi \\
& =(2 \pi h)^{-n} \int \mathrm{e}^{\mathrm{i}\langle x, \xi\rangle / h} a(x, \xi, h) \hat{u}(\xi / h) \mathrm{d} \xi
\end{aligned}
$$

We denote by $\Psi^{m}$ the set of these $\psi$ DOs. For $A \in \Psi^{m}, \sigma(A)$ will be its principal symbol.

We have $\operatorname{Op}(a): \mathscr{S}\left(\mathbb{R}^{n}\right) \rightarrow \mathscr{S}\left(\mathbb{R}^{n}\right)$ continuously and $\mathrm{Op}(a)$ can be uniquely extended to $\mathscr{S}^{\prime}\left(\mathbb{R}^{n}\right)$. Then $\operatorname{Op}(a): \mathscr{S}^{\prime}\left(\mathbb{R}^{n}\right) \rightarrow \mathscr{S}^{\prime}\left(\mathbb{R}^{n}\right)$ continuously.

Example 2.4. Consider the differential operator defined by $A=-h^{2} \Delta+V(x)+h^{2} \sum_{1 \leq j \leq n} b_{j}(x) \partial_{j}$. Its symbol and principal symbol are $a(x, \xi, h)=|\xi|^{2}+V(x)+i h \sum_{1 \leq j \leq n} b_{j}(x) \xi_{j}$ and $\sigma(A)=|\xi|^{2}+V(x)$ respectively.

We now introduce Sobolev spaces and Sobolev norms which are adapted to the scaling parameter $h$. The natural norm on $L^{2}\left(\mathbb{R}^{n}\right)$ is written as $\|u\|_{0}^{2}:=\left(\int|u(x)|^{2} \mathrm{~d} x\right)^{\frac{1}{2}}$. Let $s \in \mathbb{R}$; we then set

$$
\|u\|_{s}:=\left\|\Lambda^{s} u\right\|_{0}, \quad \text { with } \Lambda^{s}:=\operatorname{Op}\left(\langle\xi\rangle^{s}\right) \quad \text { and } \quad \mathscr{H}^{s}\left(\mathbb{R}^{n}\right):=\left\{u \in \mathscr{S}^{\prime}\left(\mathbb{R}^{n}\right) ;\|u\|_{s}<\infty\right\} .
$$

The space $\mathscr{H}^{s}\left(\mathbb{R}^{n}\right)$ is algebraically equal to the classical Sobolev space $H^{s}\left(\mathbb{R}^{n}\right)$. For a fixed value of $h$, the norm $\|\cdot\|_{s}$ is equivalent to the classical Sobolev norm that we write $\|\cdot\|_{H^{s}}$. However, these norms are not uniformly equivalent as $h$ goes to 0 . In fact we only have

$$
\|u\|_{s} \leq C\|u\|_{H^{s}}, \quad \text { if } s \geq 0, \quad \text { and } \quad\|u\|_{H^{s}} \leq C\|u\|_{s}, \quad \text { if } s \leq 0 .
$$

For $s \in \mathbb{N}$ the norm $\|\cdot\|_{s}$ is equivalent to the norm $N_{s}(u):=\sum_{|\alpha| \leq s}\left\|D^{\alpha} u\right\|_{0}^{2}=\sum_{|\alpha| \leq s} h^{2|\alpha|}\left\|\partial^{\alpha} u\right\|_{0}^{2}$. The spaces $\mathscr{H}^{s}$ and $\mathscr{H}^{-s}$ are in duality, i.e. $\mathscr{H}^{-s}=\left(\mathscr{H}^{s}\right)^{\prime}$ in the sense of distributional duality with $L^{2}=\mathscr{H}^{0}$ as a pivot space. We can prove the following continuity result.

Theorem 2.5. If $a(x, \xi, h) \in S^{m}$ and $s \in \mathbb{R}$, we then have $\mathrm{Op}(a): \mathscr{H}^{s} \rightarrow \mathscr{H}^{s-m}$ continuously, uniformly in $h$.

The following Gårding inequality is the important result we shall be interested in here. 
Theorem 2.6 (Gårding inequality). Let $K$ be a compact set of $\mathbb{R}^{n}$. If $a(x, \xi, h) \in S^{m}$, with principal part $a_{m}$, if there exists $C>0$ such that

$$
\operatorname{Re} a_{m}(x, \xi, h) \geq C\langle\xi\rangle^{m}, \quad x \in K, \xi \in \mathbb{R}^{n}, h \in\left(0, h_{0}\right),
$$

then for $0<C^{\prime}<C$ and $h_{1}>0$ sufficiently small we have

$$
\operatorname{Re}(\mathrm{Op}(a) u, u) \geq C^{\prime}\|u\|_{m / 2}^{2}, \quad u \in \mathscr{C}_{c}^{\infty}\left(\mathbb{R}^{n}\right), \operatorname{supp}(u) \subset K, 0<h \leq h_{1} .
$$

The positivity of the principal symbol of $a$ thus implies a certain positivity for the operator $\operatorname{Op}(a)$. The value of $h_{1}$ depends on $C, C^{\prime}$ and a finite number of constants $C_{\alpha, \beta}$ associated to the symbol $a(x, \xi, h)$ (see Def. 2.1). A proof of the Gårding inequality is provided in Appendix A.

Remark 2.7. We note here that the positivity condition on the principal symbol is imposed for all $\xi$ in $\mathbb{R}^{n}$, as opposed to the assumptions made for the usual Gårding inequality, i.e., non semi-classical, that only ask for such a positivity for $|\xi|$ large (see e.g. [55], Chap. 2). The semi-classical result is however stronger in the sense that it yields a true positivity for the operator.

We shall compose $\psi$ DOs in the sequel. Such compositions yield a calculus at the level of operator symbols.

Theorem 2.8 (symbol calculus). Let $a \in S^{m}$ and $b \in S^{m^{\prime}}$. Then $\operatorname{Op}(a) \circ \mathrm{Op}(b)=\operatorname{Op}(c)$ for a certain $c \in S^{m+m^{\prime}}$ that admits the following asymptotic expansion

$$
c(x, \xi, h)=(a \sharp b)(x, \xi, h) \sim \sum_{\alpha} \frac{h^{|\alpha|}}{i^{|\alpha|} \alpha !} \partial_{\xi}^{\alpha} a(x, \xi, h) \partial_{x}^{\alpha} b(x, \xi, h), \quad \text { where } \alpha !=\alpha_{1} ! \cdots \alpha_{n} \text { ! }
$$

The first term in the expansion, the principal symbol, is $a b$; the second term is $\frac{h}{i} \sum_{j} \partial_{\xi_{j}} a(x, \xi, h) \partial_{x_{j}} b(x, \xi, h)$. It follows that the principal symbol of the commutator $[\mathrm{Op}(a), \mathrm{Op}(b)]$ is

$$
\sigma([\mathrm{Op}(a), \mathrm{Op}(b)])=\frac{h}{i}\{a, b\} \in h S^{m+m^{\prime}-1} .
$$

Finally, the symbol of the adjoint operator can be obtained as follows.

Theorem 2.9. Let $a \in S^{m}$. Then $\mathrm{Op}(a)^{*}=\mathrm{Op}(b)$ for a certain $b \in S^{m}$ that admits the following asymptotic expansion

$$
b(x, \xi, h) \sim \sum_{\alpha} \frac{h^{|\alpha|}}{i^{|\alpha|} \alpha !} \partial_{\xi}^{\alpha} \partial_{x}^{\alpha} \bar{a}(x, \xi, h) .
$$

The principal symbol of $b$ is simply $\bar{a}$.

For references on usual $\psi$ DOs the reader can consult $[2,24,27,52,55]$. For references on semi-classical $\psi$ DOs the reader can consult $[13,42,50]$.

\section{Local Carleman estimates for elliptic operators}

We shall prove a local Carleman estimates for a second-order elliptic operator. To simplify notation we consider the Laplace operator $P=-\Delta$ but the method we expose extends to more general second-order elliptic operators with a principal part of the form $\sum_{i, j} \partial_{j}\left(a_{i j}(x) \partial_{i}\right)$ with $a_{i j} \in \mathscr{C}^{\infty}\left(\mathbb{R}^{n}, \mathbb{R}\right), 1 \leq i, j \leq n$ and 


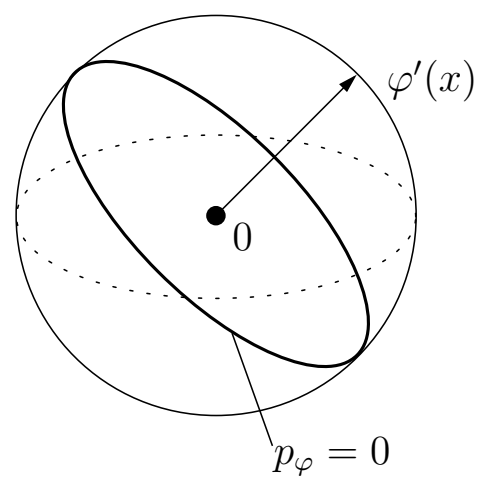

FiguRE 1. Form of the characteristic set $\mathscr{Z}$ at the vertical of each point $x \in \bar{V}$.

$\sum_{i, j} a_{i j}(x) \xi_{i} \xi_{j} \geq C|\xi|^{2}$, with $C>0$, for all $x, \xi \in \mathbb{R}^{n}$. In particular, we note that Carleman estimates are insensitive ${ }^{2}$ to changes in the operator by zero- or first-order terms.

Let $\varphi(x)$ be a real-valued function. We define the following conjugated operator $P_{\varphi}=h^{2} \mathrm{e}^{\varphi / h} P \mathrm{e}^{-\varphi / h}$ to be considered as a semi-classical differential operator. We have $P_{\varphi}=-h^{2} \Delta-\left|\varphi^{\prime}\right|^{2}+2\left\langle\varphi^{\prime}, h \nabla\right\rangle+h \Delta \varphi$. Its full symbol is given by $|\xi|^{2}-\left|\varphi^{\prime}\right|^{2}+2 i\left\langle\varphi^{\prime}, \xi\right\rangle+h \Delta \varphi$. Its principal symbol is given by

$$
p_{\varphi}=\sigma\left(P_{\varphi}\right)=|\xi|^{2}-\left|\varphi^{\prime}\right|^{2}+2 i\left\langle\varphi^{\prime}, \xi\right\rangle=\sum_{j}\left(\xi_{j}+i \varphi_{x_{j}}^{\prime}\right)^{2},
$$

i.e., we have "replaced" $\xi_{j}$ by $\xi_{j}+i \varphi_{x_{j}}^{\prime}$. In fact we note that the symbol of $\mathrm{e}^{\varphi / h} D_{j} \mathrm{e}^{-\varphi / h}$ is $\xi_{j}+i \varphi_{x_{j}}^{\prime}$.

We define the following symmetric operators $Q_{2}=\left(P_{\varphi}+P_{\varphi}^{*}\right) / 2, Q_{1}=\left(P_{\varphi}-P_{\varphi}^{*}\right) /(2 i)$, with respective principal symbols

We have $p_{\varphi}=q_{2}+i q_{1}$ and $P_{\varphi}=Q_{2}+i Q_{1}$.

$$
q_{2}=|\xi|^{2}-\left|\varphi^{\prime}\right|^{2}, \quad q_{1}=2\left\langle\xi, \varphi^{\prime}\right\rangle .
$$

We choose $\varphi$ that satisfies the following assumption.

Assumption 3.1 (Hörmander $[25,26]$ ). Let $V$ be a bounded open set in $\mathbb{R}^{n}$. We say that the weight function $\varphi \in \mathscr{C}^{\infty}\left(\mathbb{R}^{n}, \mathbb{R}\right)$ satisfies the sub-ellipticity assumption in $\bar{V}$ if $\left|\varphi^{\prime}\right|>0$ in $\bar{V}$ and

$$
\forall(x, \xi) \in \bar{V} \times \mathbb{R}^{n}, \quad p_{\varphi}(x, \xi)=0 \quad \Rightarrow \quad\left\{q_{2}, q_{1}\right\}(x, \xi) \geq C>0 .
$$

Remark 3.2. We note that $p_{\varphi}(x, \xi)=0$ is equivalent to $|\xi|=\left|\varphi^{\prime}\right|$ and $\left\langle\xi, \varphi^{\prime}\right\rangle=0$. In particular, the characteristic set $\mathscr{Z}=\left\{(x, \xi) \in \bar{V} \times \mathbb{R}^{n} ; p_{\varphi}(x, \xi)=0\right\}$ is compact as illustrated in Figure 1 .

Assumption 3.1 can be fulfilled as stated in the following lemma whose proof can be found in Appendix A.

Lemma 3.3 (Hörmander $[25,26]$ ). Let $V$ be a bounded open set in $\mathbb{R}^{n}$ and $\psi \in \mathscr{C}^{\infty}\left(\mathbb{R}^{n}, \mathbb{R}\right)$ be such that $\left|\psi^{\prime}\right|>0$ in $\bar{V}$. Then $\varphi=\mathrm{e}^{\lambda \psi}$ fulfills Assumption 3.1 in $\bar{V}$ for $\lambda>0$ sufficiently large.

The proof of the Carleman estimate will make use of the Gårding inequality. In preparation, we have the following result proven in Appendix A that follows from Assumption 3.1.

Lemma 3.4. Let $\mu>0$ and $\rho=\mu\left(q_{2}^{2}+q_{1}^{2}\right)+\left\{q_{2}, q_{1}\right\}$. Then, for all $(x, \xi) \in \bar{V} \times \mathbb{R}^{n}$, we have $\rho(x, \xi) \geq C\langle\xi\rangle^{4}$, with $C>0$, for $\mu$ sufficiently large.

We may now prove the following Carleman estimate.

\footnotetext{
${ }^{2}$ In the sense that only constants are affected. In Theorem 3.5 below the constants $C$ and $h_{1}$ change but not the form of the estimate.
} 
Theorem 3.5. Let $V$ be a bounded open set in $\mathbb{R}^{n}$ and let $\varphi$ satisfy Assumption 3.1 in $\bar{V}$; then, there exist $0<h_{1}<h_{0}$ and $C>0$ such that

$$
h\left\|\mathrm{e}^{\varphi / h} u\right\|_{0}^{2}+h^{3}\left\|\mathrm{e}^{\varphi / h} \nabla_{x} u\right\|_{0}^{2} \leq C h^{4}\left\|\mathrm{e}^{\varphi / h} P u\right\|_{0}^{2},
$$

for $u \in \mathscr{C}_{c}^{\infty}(\bar{V})$ and $0<h<h_{1}$.

Proof We set $v=\mathrm{e}^{\varphi / h} u$. Then, $P u=f$ is equivalent to $P_{\varphi} v=g=h^{2} \mathrm{e}^{\varphi / h} f$ or rather $Q_{2} v+i Q_{1} v=g$. Observing that $\left(Q_{j} w_{1}, w_{2}\right)=\left(w_{1}, Q_{j} w_{2}\right)$ for $w_{1}, w_{2} \in \mathscr{C}_{c}^{\infty}\left(\mathbb{R}^{n}\right)$ we then obtain

$$
\|g\|_{0}^{2}=\left\|Q_{1} v\right\|_{0}^{2}+\left\|Q_{2} v\right\|_{0}^{2}+2 \operatorname{Re}\left(Q_{2} v, i Q_{1} v\right)=\left(\left(Q_{1}^{2}+Q_{2}^{2}+i\left[Q_{2}, Q_{1}\right]\right) v, v\right) .
$$

We choose $\mu>0$ as given in Lemma 3.4. Then, for $h$ such that $h \mu \leq 1$ we have

$$
h(\underbrace{\left(\mu\left(Q_{1}^{2}+Q_{2}^{2}\right)+\frac{i}{h}\left[Q_{2}, Q_{1}\right]\right)}_{\text {principal symbol }=\mu\left(q_{1}^{2}+q_{2}^{2}\right)+\left\{q_{2}, q_{1}\right\}} v, v) \leq\|g\|_{0}^{2} .
$$

The Gårding inequality and Lemma 3.4 then yield

$$
h\|v\|_{2}^{2} \leq C\|g\|_{0}^{2} .
$$

We content ${ }^{3}$ ourselves with the norm in $\mathscr{H}^{1}$ here and we obtain $h\left\|\mathrm{e}^{\varphi / h} u\right\|_{0}^{2}+h^{3}\left\|\nabla_{x}\left(\mathrm{e}^{\varphi / h} u\right)\right\|_{0}^{2} \leq C h^{4}\left\|\mathrm{e}^{\varphi / h} f\right\|_{0}^{2}$. We write $\nabla_{x}\left(\mathrm{e}^{\varphi / h} u\right)=h^{-1} \mathrm{e}^{\varphi / h}\left(\nabla_{x} \varphi\right) u+\mathrm{e}^{\varphi / h} \nabla_{x} u$, which yields

$$
h^{3}\left\|\mathrm{e}^{\varphi / h} \nabla_{x} u\right\|_{0}^{2} \leq C h\left\|\mathrm{e}^{\varphi / h} u\right\|_{0}^{2}+C h^{3}\left\|\nabla_{x}\left(\mathrm{e}^{\varphi / h} u\right)\right\|_{0}^{2},
$$

since $\left|\nabla_{x} \varphi\right| \leq C$. This concludes the proof.

Remark 3.6. With a density argument the result of Theorem 3.5 can be extended to functions $u \in H_{0}^{2}(V)$. However, here, we do not treat the case of functions in $H_{0}^{1}(V) \cap H^{2}(V)$. For such a result one needs a local Carleman estimate at the boundary of the open set $V$ as proven in [39], Proposition 2, page 351. Moreover, a global estimate in $V$ for a function $u$ in $H_{0}^{1}(V) \cap H^{2}(V)$ requires an observation term in the r.h.s. of the Carleman estimate. We shall provide such details in the case of parabolic operators below (see Sect. 7).

Remark 3.7. In the proof of Theorem 3.5 we have used Assumption 3.1. We give complementary roles to the square terms in (3.2), $\left\|Q_{1} u\right\|_{0}^{2}$ and $\left\|Q_{2} u\right\|_{0}^{2}$, and to the action of the commutator $i\left(\left[Q_{2}, Q_{1}\right] u, u\right)$. As the square terms approach zero, the commutator term comes into effect and yields positivity. A. Fursikov and Imanuvilov [22] have introduced a modification of the proof that allows to only consider a term equivalent to the commutator term without using the two square terms. This approach is presented below.

The following proposition yields a more precise result than the previous Carleman estimate and illustrates the loss of a half derivative in the neighborhood of the characteristic set $\mathscr{Z}$.

Proposition 3.8. Let $s \in \mathbb{R}$ and $V$ be a bounded open set in $\mathbb{R}^{n}$ and let $\varphi$ satisfy Assumption 3.1 in $\bar{V}$. Let $\chi_{1}, \chi_{2} \in S^{0}$ with compact supports in $x$. Assume that $\chi_{1}$ vanishes in a neighborhood of $\mathscr{Z}$ and that $\chi_{2}$ vanishes

\footnotetext{
${ }^{3}$ Note that in the elliptic region, e.g. for large $|\xi|$, we can obtain a better result without the factor $h$ in (3.3). In the neighborhood of the characteristic set $\mathscr{Z}=\left\{p_{\varphi}=0\right\}$ the choice of the norm in $\mathscr{H}^{1}$ or $\mathscr{H}^{2}$ matters very little since this region is compact. See Proposition 3.8 for more details.
} 


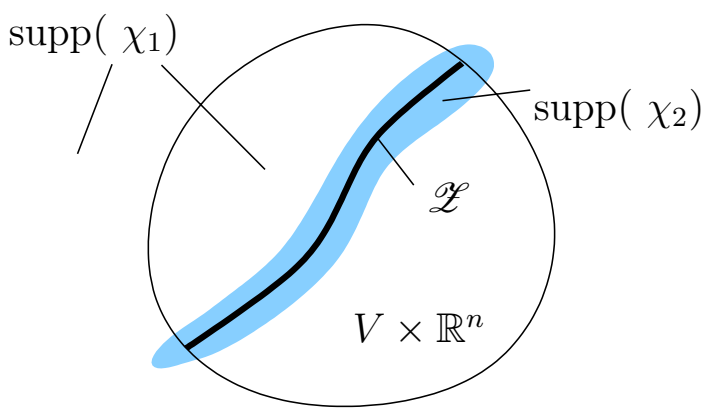

Figure 2. Characteristic set $\mathscr{Z}$ and supports of $\chi_{1}$ and $\chi_{2}$ in Proposition 3.8.

outside a compact neighborhood of $\mathscr{Z}$. Then there exist $C>0$ and $0<h_{2}<h_{0}$ such that

$$
\left\|\mathrm{Op}\left(\chi_{1}\right) v\right\|_{2} \leq C\left(\left\|P_{\varphi} v\right\|_{0}+h\|v\|_{1}\right), \quad \text { and } h^{\frac{1}{2}}\left\|\mathrm{Op}\left(\chi_{2}\right) v\right\|_{s} \leq C\left(\left\|P_{\varphi} v\right\|_{0}+h\|v\|_{1}\right),
$$

for $v \in \mathscr{C}_{c}^{\infty}(\bar{V})$ and $0<h<h_{2}$.

The geometry of the cut-off functions in Proposition 3.8 is illustrated in Figure 2. This proposition is proven in Appendix A.5. We take $\chi_{1}$ and $\chi_{2}$ that satisfy the assumptions made in Proposition 3.8 and such that $\chi_{1}+\chi_{2}=1$ in a neighborhood of $\bar{V} \times \mathbb{R}^{n}$. For $v \in \mathscr{C}_{c}^{\infty}(\bar{V})$ we have $\left\|\mathrm{Op}\left(1-\chi_{1}-\chi_{2}\right) v\right\|_{r} \leq C_{N, r, r^{\prime}} h^{N}\|v\|_{r^{\prime}}$ for all $N \in \mathbb{N}$ and $r, r^{\prime} \in \mathbb{R}$. We thus obtain

$$
h^{\frac{1}{2}}\|v\|_{2} \leq h^{\frac{1}{2}}\left(\left\|\mathrm{Op}\left(1-\chi_{1}-\chi_{2}\right) v\right\|_{2}+\left\|\mathrm{Op}\left(\chi_{1}\right) v\right\|_{2}+\left\|\mathrm{Op}\left(\chi_{2}\right) v\right\|_{2}\right) \leq C\left(\left\|P_{\varphi} v\right\|_{0}+h\|v\|_{1}\right) .
$$

Choosing $h$ sufficiently small we obtain

$$
h^{\frac{1}{2}}\|v\|_{2} \leq C^{\prime}\left\|P_{\varphi} v\right\|_{0}
$$

which brings us back to the last step in the proof of Theorem 3.5. Also note that (3.5) allows us to remove the second term in the r.h.s. in each inequalities in (3.4) and we thus obtain

$$
\left\|\mathrm{Op}\left(\chi_{1}\right) v\right\|_{2} \leq C\left\|P_{\varphi} v\right\|_{0}, \quad \text { and } h^{\frac{1}{2}}\left\|\mathrm{Op}\left(\chi_{2}\right) v\right\|_{s} \leq C\left\|P_{\varphi} v\right\|_{0} .
$$

We have seen that the sub-ellipticity condition in Assumption 3.1 is sufficient to obtain a Carleman estimate in Theorem 3.5. In fact we can prove that this condition is necessary. We also note that the powers of the factors $h$ in the l.h.s. of the estimate in Theorem 3.5 as well as in the second inequality in (3.6) are optimal: for instance, we may not have $h^{2 \alpha}$ in front of the first term in inequality (3.1) with $\alpha<\frac{1}{2}$. These two points are summarized in the following proposition.

Proposition 3.9. Let $V$ be a bounded open set in $\mathbb{R}^{n}, \varphi(x) \in \mathscr{C}^{\infty}\left(\mathbb{R}^{n}, \mathbb{R}\right), 0<h_{1}<h_{0}$ and $C>0$ such that for a certain $\alpha \leq \frac{1}{2}$ we have

$$
h^{\alpha}\left\|\mathrm{e}^{\varphi / h} u\right\|_{0} \leq C h^{2}\left\|\mathrm{e}^{\varphi / h} P u\right\|_{0},
$$

for all $u \in \mathscr{C}_{c}^{\infty}(V)$ and $0<h<h_{1}$. Then $\alpha=\frac{1}{2}$ and the weight function $\varphi$ satisfies Assumption 3.1 in $\bar{V}$.

The reader is referred to Appendix A.6 for a proof. 


\subsection{The method of Fursikov and Imanuvilov}

Following the approach introduced by Fursikov and Imanuvilov [22], we provide an alternative proof of Theorem 3.5 in the elliptic case. We use the notation of the proof of Theorem 3.5, and write

$$
\|g+\mu h \Delta \varphi v\|_{0}^{2}=\left\|Q_{2} v\right\|_{0}^{2}+\left\|\tilde{Q}_{1} v\right\|_{0}^{2}+\left(i\left[Q_{2}, Q_{1}\right] v, v\right)+2 \operatorname{Re}\left(Q_{2} v, \mu h \Delta \varphi v\right), \quad 0<\mu<2
$$

where $\tilde{Q}_{1}=Q_{1}-i \mu h \Delta \varphi$ and we obtain $\|g+\mu h \Delta \varphi v\|_{0}^{2}=\left\|Q_{2} v\right\|_{0}^{2}+\left\|\tilde{Q}_{1} v\right\|_{0}^{2}+h \operatorname{Re}(R v, v)$, where $\rho=\sigma(R)=$ $\left(\left\{q_{2}, q_{1}\right\}+2 \mu q_{2} \Delta \varphi\right)$. We have the following lemma, which proof can be found in Section A.4.

Lemma 3.10. If $\varphi=\mathrm{e}^{\lambda \psi}$, then for $\lambda>0$ sufficiently large, there exists $C_{\lambda}>0$ such that

$$
\rho=\left\{q_{2}, q_{1}\right\}+2 \mu q_{2} \Delta \varphi \geq C_{\lambda}\langle\xi\rangle^{2}, \quad x \in \bar{V}, \xi \in \mathbb{R}^{n}
$$

With the Gårding inequality we then conclude that $\operatorname{Re}(R v, v) \geq C^{\prime}\|v\|_{1}^{2}$, for $0<C^{\prime}<C_{\lambda}$ and $h$ taken sufficiently small. The Carleman estimate follows without using the square terms $\left\|Q_{2} v\right\|_{0}^{2}$ and $\left\|\tilde{Q}_{1} v\right\|_{0}^{2}$. In fact we write

$$
\|g+\mu h \Delta \varphi v\|_{0}^{2} \leq 2\|g\|_{0}^{2}+2 \mu^{2} h^{2}\|\Delta \varphi v\|_{0}^{2}
$$

and the second term in the r.h.s. can be "absorbed" by $h\|v\|_{1}^{2}$ for $h$ sufficiently small.

Remark 3.11. The method of Fursikov and Imanuvilov, at the symbol level, is a matter of adding a term of the form $2 \mu q_{2} \Delta \varphi$ to the commutator symbol $\frac{i}{h}\left[Q_{2}, Q_{1}\right]$. As the sign of $q_{2} \Delta \varphi$ is not fixed, a precise choice of the value of $\mu$ is crucial.

In the proof of Lemma 3.3 in Section A.2 we in fact obtained the following condition on the weight function

$$
\forall(x, \xi) \in \bar{V} \times \mathbb{R}^{n}, \quad q_{2}(x, \xi)=0 \Rightarrow\left\{q_{2}, q_{1}\right\}(x, \xi) \geq C>0,
$$

which is stronger that the condition in Assumption 3.1, which reads

$$
\forall(x, \xi) \in \bar{V} \times \mathbb{R}^{n}, \quad p_{\varphi}(x, \xi)=0 \quad \Rightarrow \quad\left\{q_{2}, q_{1}\right\}(x, \xi) \geq C>0 .
$$

Finally the condition of Fursikov and Imanuvilov, i.e., $\left\{q_{2}, q_{1}\right\}+2 \mu q_{2} \Delta \varphi \geq C\langle\xi\rangle^{2}$ is itself stronger than (3.8). The different conditions we impose on the weight function $\varphi$ are sufficient to derive a Carleman estimate. We recall that the weaker condition, that of Assumption 3.1, is in fact necessary (see Prop. 3.9 and its proof in Sect. A.6).

Condition (3.8) turns out to be useful in some situations, in particular to prove Carleman estimates for parabolic operators, such as $\partial_{t}-\Delta$, as it is done in Section 7.1.

\section{UNiQUe CONTINUATION}

Let $\Omega$ be a bounded open set in $\mathbb{R}^{n}$. In a neighborhood $V$ of a point $x_{0} \in \Omega$, we take a function $f$ such that $\nabla f \neq 0$ in $\bar{V}$. Let $p(x, \xi)$ be a second-order polynomial in $\xi$ that satisfies $p(x, \xi) \geq C|\xi|^{2}$ with $C>0$. We define the differential operator $P=p(x, \partial / i)$.

We consider $u \in H^{2}(V)$ solution of $P u=g(u)$, where $g$ is such that $|g(y)| \leq C|y|, y \in \mathbb{R}$. We assume that $u=0$ in $\left\{x \in V ; f(x) \geq f\left(x_{0}\right)\right\}$. We aim to show that the function $u$ vanishes in a neighborhood of $x_{0}$.

We pick a function $\psi$ whose gradient does not vanish near $\bar{V}$ and that satisfies $\left\langle\nabla f\left(x_{0}\right), \nabla \psi\left(x_{0}\right)\right\rangle>0$ and is such that $f-\psi$ reaches a strict local minimum at $x_{0}$ as one moves along the level set $\left\{x \in V ; \psi(x)=\psi\left(x_{0}\right)\right\}$. For instance, we may choose $\psi(x)=f(x)-c\left|x-x_{0}\right|^{2}$. We then set $\varphi=\mathrm{e}^{\lambda \psi}$ according to Lemma 3.3. In the neighborhood $V$ (or possibly in a smaller neighborhood of $x_{0}$ ) the geometrical situation we have just described is illustrated in Figure 3. 


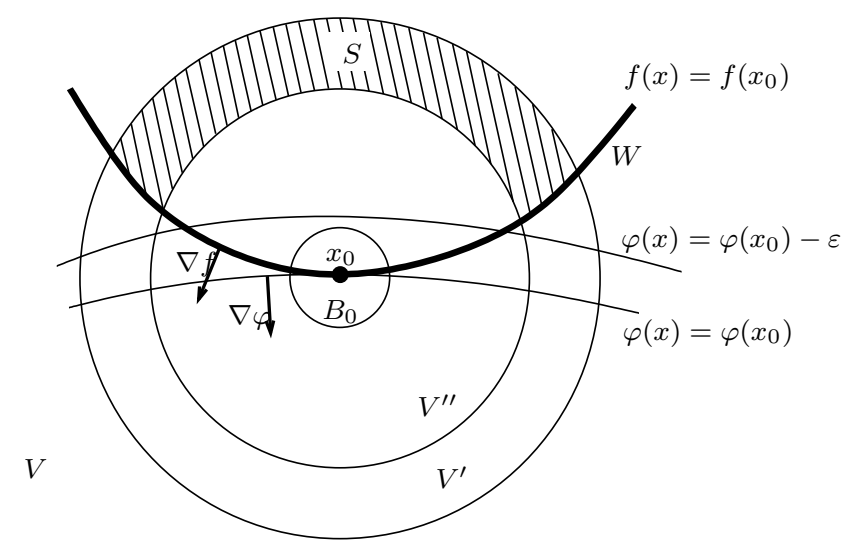

Figure 3. Local geometry for the unique continuation problem. The striped region contains the support of $[P, \chi] u$.

We call $W$ the region $\left\{x \in V ; f(x) \geq f\left(x_{0}\right)\right\}$ (region beneath $\left\{f(x)=f\left(x_{0}\right)\right\}$ in Fig. 3). We choose $V^{\prime}$ and $V^{\prime \prime}$ neighborhoods of $x_{0}$ such that $V^{\prime \prime} \Subset V^{\prime} \Subset V$ and we pick a function $\chi \in \mathscr{C}_{c}^{\infty}\left(V^{\prime}\right)$ such that $\chi=1$ in $V^{\prime \prime}$. We set $v=\chi u$ and then $v \in H_{0}^{2}(V)$. Observe that the Carleman estimate of Theorem 3.5 applies to $v$ by Remark 3.6. We have

$$
P v=P(\chi u)=\chi P u+[P, \chi] u,
$$

where the commutator is a first-order differential operator. We thus obtain

$$
\begin{aligned}
h\left\|\mathrm{e}^{\varphi / h} \chi u\right\|_{0}^{2}+h^{3}\left\|\mathrm{e}^{\varphi / h} \nabla_{x}(\chi u)\right\|_{0}^{2} & \leq C\left(h^{4}\left\|\mathrm{e}^{\varphi / h} \chi g(u)\right\|_{0}^{2}+h^{4}\left\|\mathrm{e}^{\varphi / h}[P, \chi] u\right\|_{0}^{2}\right) \\
& \leq C^{\prime}\left(h^{4}\left\|\mathrm{e}^{\varphi / h} \chi u\right\|_{0}^{2}+h^{4}\left\|\mathrm{e}^{\varphi / h}[P, \chi] u\right\|_{0}^{2}\right), \quad 0<h<h_{1} .
\end{aligned}
$$

Choosing $h$ sufficiently small, say $h<h_{2}$, we may ignore the first term in the r.h.s. of the previous estimate. We then write

$h\left\|\mathrm{e}^{\varphi / h} u\right\|_{L^{2}\left(V^{\prime \prime}\right)}^{2}+h^{3}\left\|\mathrm{e}^{\varphi / h} \nabla_{x} u\right\|_{L^{2}\left(V^{\prime \prime}\right)}^{2} \leq h\left\|\mathrm{e}^{\varphi / h} \chi u\right\|_{0}^{2}+h^{3}\left\|\mathrm{e}^{\varphi / h} \nabla_{x}(\chi u)\right\|_{0}^{2} \leq C h^{4}\left\|\mathrm{e}^{\varphi / h}[P, \chi] u\right\|_{L^{2}(S)}^{2}, 0<h<h_{2}$,

where $S:=V^{\prime} \backslash\left(V^{\prime \prime} \cup W\right)$, since the support of $[P, \chi] u$ is confined in the region where $\chi$ varies and $u$ does not vanish (see the striped region in Fig. 3).

For all $\varepsilon \in \mathbb{R}$, we set $V_{\varepsilon}=\left\{x \in V ; \varphi(x) \leq \varphi\left(x_{0}\right)-\varepsilon\right\}$. There exists $\varepsilon>0$ such that $S \Subset V_{\varepsilon}$. We then choose a ball $B_{0}$ with center $x_{0}$ such that $B_{0} \subset V^{\prime \prime} \backslash V_{\varepsilon}$ and obtain

$$
\mathrm{e}^{\inf _{B_{0}} \varphi / h}\|u\|_{H^{1}\left(B_{0}\right)} \leq C \mathrm{e}^{\sup _{S} \varphi / h}\|u\|_{H^{1}(S)}, \quad 0<h<h_{2}
$$

Since $\inf _{B_{0}} \varphi>\sup _{S} \varphi$, letting $h$ go to zero, we obtain $u=0$ in $B_{0}$. We have thus proven the following local unique-continuation result.

Proposition 4.1. Let $g$ be such that $|g(y)| \leq C|y|, x_{0} \in \Omega$ and $u \in H_{\mathrm{loc}}^{2}(\Omega)$ satisfying Pu=g(u) and $u=0$ in $\left\{x ; f(x) \geq f\left(x_{0}\right)\right\}$, in a neighborhood $V$ of $x_{0}$. The function $f$ is defined in $V$ and such that $|\nabla f| \neq 0$ in a neighborhood of $x_{0}$. Then $u$ vanishes in a neighborhood of $x_{0}$.

With a connectedness argument we then prove the following theorem.

Theorem 4.2 (Calderón theorem). Let $g$ be such that $|g(y)| \leq C|y|$. Let $\Omega$ be an connected open set in $\mathbb{R}^{n}$ and let $\omega \Subset \Omega$, with $\omega \neq \emptyset$. If $u \in H^{2}(\Omega)$ satisfies $P u=g(u)$ in $\Omega$ and $u(x)=0$ in $\omega$, then $u$ vanishes in $\Omega$. 
Proof The support of $u$ is a closed set. Since $F=\operatorname{supp}(u)$ cannot be equal to $\Omega$, let us show that $F$ is open. It will then follow that $F=\emptyset$. Assume that $\operatorname{fr}(F)=F \backslash F^{\circ}$ is not empty and chose $x_{1} \in \operatorname{fr}(F)$. We set $A:=\Omega \backslash F$. We recall that we denote by $B(x, r)$ the Euclidean open ball with center $x$ and radius $r$. There exists $R>0$ such that $B\left(x_{1}, R\right) \Subset \Omega$ and $x_{0} \in B\left(x_{1}, R / 4\right)$ such that $x_{0} \in A$. Since $A$ is open, there exists $0<r_{1}<R / 2$ such that $B\left(x_{0}, r_{1}\right) \subset A$. For $r_{2}=R / 2$ we have thus obtained $r_{1}<r_{2}$ such that

$$
B\left(x_{0}, r_{1}\right) \subset A, \quad B\left(x_{0}, r_{2}\right) \Subset \Omega, \quad \text { and } x_{1} \in B\left(x_{0}, r_{2}\right) .
$$

We set $\mathscr{B}_{t}=B\left(x_{0},(1-t) r_{1}+t r_{2}\right)$ for $0 \leq t \leq 1$. The previous proposition shows that is $u$ vanishes in $\mathscr{B}_{t}$, with $0 \leq t \leq 1$, then there exists $\varepsilon>0$ such that $u$ vanishes in $B_{t+\varepsilon}$. Since $u$ vanishes in $\mathscr{B}_{0}$, we thus find that $u$ vanishes in $\mathscr{B}_{1}$, and in particular in a neighborhood of $x_{0}$ that thus cannot be in $\operatorname{fr}(F)$. Hence $F$ is open.

\section{INTERPOLATION AND SPECTRAL INEQUALITIES}

Let $\Omega$ be a bounded open set in $\mathbb{R}^{n}, S_{0}>0$ and $\alpha \in\left(0, S_{0} / 2\right)$. Let also $Z=\left(0, S_{0}\right) \times \Omega$ and $Y=\left(\alpha, S_{0}-\alpha\right) \times \Omega$. We set $z=(s, x)$ with $s \in\left(0, S_{0}\right)$ and $x \in \Omega$. We define the elliptic operator $A:=-\partial_{s}^{2}-\Delta_{x}$ in $Z$. The Carleman estimate that we have proven in Section 3 holds for this operator.

We start with a weight function $\varphi(z)$ defined in $Z$ and choose $\rho_{1}<\rho_{1}^{\prime}<\rho_{2}<\rho_{2}^{\prime}<\rho_{3}<\rho_{3}^{\prime}$ and set

$$
V=\left\{z \in Z ; \rho_{1}<\varphi(z)<\rho_{3}^{\prime}\right\}, \quad V_{j}=\left\{z \in Z ; \rho_{j}<\varphi(z)<\rho_{j}^{\prime}\right\}, \quad j=1,2,3 .
$$

We assume that $\bar{V}$ is compact in $Z$ (we remain away from the boundary of $Z$ ) and that $\varphi$ satisfies the subellipticity Assumption 3.1 in $\bar{V}$.

The Carleman estimate of Theorem 3.5 yields the following local interpolation inequality.

Proposition 5.1 (Lebeau-Robbiano [39]). There exist $C>0$ and $\delta_{0} \in(0,1)$ such that for $u \in H^{2}(V)$ we have

$$
\|u\|_{H^{1}\left(V_{2}\right)} \leq C\left(\|A u\|_{L^{2}(V)}+\|u\|_{H^{1}\left(V_{3}\right)}\right)^{\delta}\|u\|_{H^{1}(V)}^{1-\delta},
$$

for $\delta \in\left[0, \delta_{0}\right]$.

Proof Let $\chi \in \mathscr{C}_{c}^{\infty}(V)$ be such that $\chi(z)=1$ in a neighborhood of $\rho_{1}^{\prime} \leq \varphi(z) \leq \rho_{3}$. We set $w=\chi u$. The Carleman estimate of Theorem 3.5 implies $\left\|\mathrm{e}^{\varphi / h} w\right\|_{0}+\left\|\mathrm{e}^{\varphi / h} \nabla w\right\|_{0} \leq C\left\|\mathrm{e}^{\varphi / h} A w\right\|_{0}$ for $h$ small, $0<h<h_{1} \leq 1$. We then observe that $A w=\chi A u+[A, \chi] u$, with the first-order operator $[A, \chi]$ uniquely supported in $V_{1} \cup V_{3}$. We thus obtain

$$
\mathrm{e}^{\rho_{2} / h}\|u\|_{H^{1}\left(V_{2}\right)} \leq C \mathrm{e}^{\rho_{1}^{\prime} / h}\|u\|_{H^{1}\left(V_{1}\right)}+C \mathrm{e}^{\rho_{3}^{\prime} / h}\left(\|A u\|_{L^{2}(V)}+\|u\|_{H^{1}\left(V_{3}\right)}\right),
$$

as $\chi=1$ in $V_{2}$. We finally write

$$
\mathrm{e}^{\rho_{2} / h}\|u\|_{H^{1}\left(V_{2}\right)} \leq C \mathrm{e}^{\rho_{1}^{\prime} / h}\|u\|_{H^{1}(V)}+C \mathrm{e}^{\rho_{3}^{\prime} / h}\left(\|A u\|_{L^{2}(V)}+\|u\|_{H^{1}\left(V_{3}\right)}\right), \quad 0<h \leq h_{1} .
$$

We conclude with the following lemma.

Lemma 5.2 (Robbiano [49]). Let $C_{1}, C_{2}$ and $C_{3}$ be positive and $A, B, C$ non negative, such that $C \leq C_{3} A$ and such that for all $\gamma \geq \gamma_{0}$ we have

$$
C \leq \mathrm{e}^{-C_{1} \gamma} A+\mathrm{e}^{C_{2} \gamma} B
$$

Then

$$
C \leq \operatorname{Cst} A^{\frac{C_{2}}{C_{1}+C_{2}}} B^{\frac{C_{1}}{C_{1}+C_{2}}}
$$




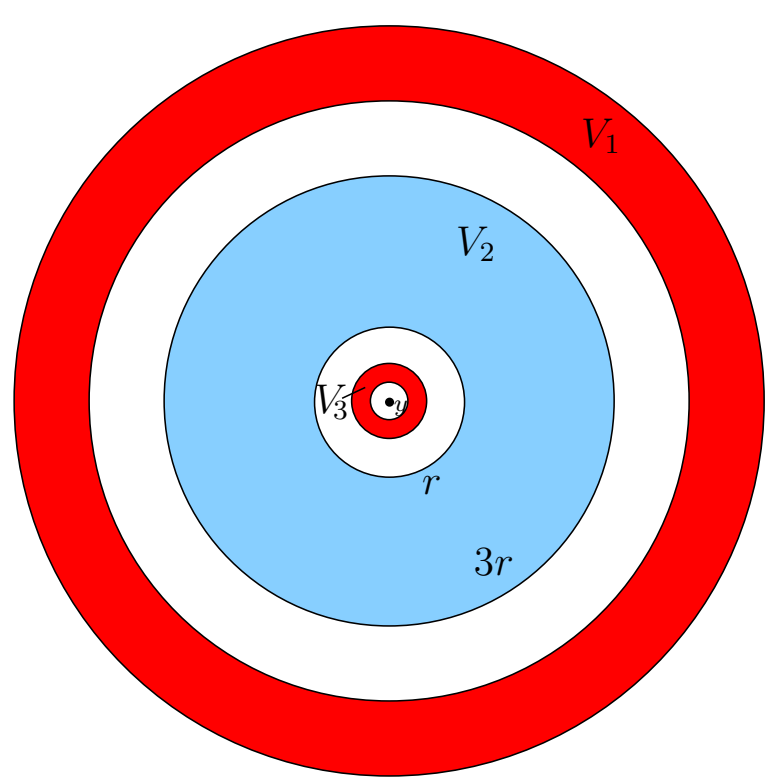

Figure 4. Level sets of the weight function $\varphi$ and regions $V_{1}, V_{2}$ and $V_{3}$. The red regions, $V_{1}$ and $V_{3}$, localise the support of $\nabla \chi$.

Proof We optimize the r.h.s. of (5.1) as a function of $\gamma$ and we find $\gamma_{\mathrm{opt}}=\frac{\ln \left(\left(A C_{1}\right) /\left(B C_{2}\right)\right)}{C_{1}+C_{2}}$. To simplify we choose $\gamma_{1}=\frac{\ln (A / B)}{C_{1}+C_{2}}$. If $\gamma_{1} \geq \gamma_{0}$, substitution in (5.1) then yields (5.2). If we now have $\gamma_{1}<\gamma_{0}$, we then see that $A \leq \operatorname{Cst} B$. We conclude as $C \leq C_{3} A$.

We now apply the result of Proposition 5.1 to a particular weight function. Let $y \in Z$ and $r>0$ be such that $B(y, 6 r) \Subset Z$. Let us set $\psi(z)=-\operatorname{dist}(z, y)$ and choose $\lambda>0$ such that $\varphi=\mathrm{e}^{\lambda \psi}$ satisfies the sub-ellipticity Assumption 3.1 in $B(y, 6 r) \backslash B(y, r / 8)$ by Lemma 3.3. We then take

$$
\rho_{1}=\mathrm{e}^{-5 r \lambda}, \rho_{1}^{\prime}=\mathrm{e}^{-4 r \lambda}, \rho_{2}=\mathrm{e}^{-3 r \lambda}, \rho_{2}^{\prime}=\mathrm{e}^{-r \lambda}, \rho_{3}=\mathrm{e}^{-\frac{r}{2} \lambda}, \rho_{3}^{\prime}=\mathrm{e}^{-\frac{r}{4} \lambda} .
$$

The neighborhoods $V_{1}, V_{2}$ and $V_{3}$ are illustrated in Figure 4.

By applying Proposition 5.1 we obtain, for $u \in H^{2}(Z)$,

$$
\|u\|_{H^{1}\left(V_{2}\right)} \leq C\left(\|A u\|_{L^{2}(V)}+\|u\|_{H^{1}\left(V_{3}\right)}\right)^{\delta}\|u\|_{H^{1}(V)}^{1-\delta} \leq C\left(\|A u\|_{L^{2}(Z)}+\|u\|_{H^{1}(B(y, r))}\right)^{\delta}\|u\|_{H^{1}(Z)}^{1-\delta},
$$

which yields

$$
\|u\|_{H^{1}(B(y, 3 r))} \leq C\left(\|A u\|_{L^{2}(Z)}+\|u\|_{H^{1}(B(y, r))}\right)^{\delta}\|u\|_{H^{1}(Z)}^{1-\delta} .
$$

The $H^{1}$-norm in the ball $B(y, 3 r)$ is thus estimated by the $H^{1}$-norm in the ball $B(y, r)$. In particular, we recover the local uniqueness result of Section 4 when $A u=0$.

When $\Omega$ has a regular boundary, this local inequality can be "propagated" up to the boundary, under suitable boundary conditions. We then obtain a global result. In addition to the Carleman estimate we have proven here, one needs to prove a similar estimate at the boundary $\left(0, S_{0}\right) \times \partial \Omega$. The proof we give below of a Carleman estimate at the boundary for a parabolic operator (see Thm. 7.6 and its proof in Appendix A.10) is similar to the proof of a Carleman estimate at the boundary for an elliptic operator (see [39]). The "propagation" technique makes use of a finite covering by balls of radius $r$. The reader is referred to [39] for details (pp. 353-356). Here, as in [41] (see the proof of Theorem 3, pp. 312-313), the interpolation inequality can be "initiated" at the boundary $s=0$ (again by a Carleman estimate at the boundary). 
Theorem $5.3([31,39,41])$. Let $\omega$ be an open set in $\Omega$. There exist $C>0$ and $\delta \in(0,1)$ such that for $u \in H^{2}(Z)$ that satisfies $\left.u(s, x)\right|_{x \in \partial \Omega}=0$, for $s \in\left(0, S_{0}\right)$ and $u(0, x)=0$, for $x \in \Omega$, we have

$$
\|u\|_{H^{1}(Y)} \leq C\|u\|_{H^{1}(Z)}^{1-\delta}\left(\|A u\|_{L^{2}(Z)}+\left\|\partial_{s} u(0, x)\right\|_{L^{2}(\omega)}\right)^{\delta} .
$$

We may now deduce a spectral inequality that, in particular, measures the loss of orthogonality of the eigenfunctions of $-\Delta$ in $\Omega$, with homogeneous Dirichlet boundary conditions, when they are restricted to an open subset $\omega \subset \Omega$ such that $\bar{\omega} \neq \Omega$. Let $\phi_{j}, j \in \mathbb{N}^{*}$, be an orthonormal basis of such eigenfunctions and $\mu_{1} \leq \mu_{2} \leq \cdots \leq \mu_{k} \leq \cdots$ the associated eigenvalues, counted with their multiplicity.

Theorem $5.4([31,41])$. There exists $K>0$ such that for all sequences $\left(\alpha_{j}\right)_{j \in \mathbb{N}^{*}} \subset \mathbb{C}$ and all $\mu>0$ we have

$$
\sum_{\mu_{j} \leq \mu}\left|\alpha_{j}\right|^{2}=\int_{\Omega}\left|\sum_{\mu_{j} \leq \mu} \alpha_{j} \phi_{j}(x)\right|^{2} \mathrm{~d} x \leq K \mathrm{e}^{K \sqrt{\mu}} \int_{\omega}\left|\sum_{\mu_{j} \leq \mu} \alpha_{j} \phi_{j}(x)\right|^{2} \mathrm{~d} x
$$

or concisely $\left\|\sum_{\mu_{j} \leq \mu} \alpha_{j} \phi_{j}\right\|_{L^{2}(\Omega)}^{2} \leq K \mathrm{e}^{K \sqrt{\mu}}\left\|\sum_{\mu_{j} \leq \mu} \alpha_{j} \phi_{j}\right\|_{L^{2}(\omega)}^{2}$.

Proof We apply Inequality (5.3) to the function $u(s, x)=\sum_{\mu_{j} \leq \mu} \alpha_{j} \frac{\sinh \left(\sqrt{\mu_{j}} s\right)}{\sqrt{\mu_{j}}} \phi_{j}(x)$ that satisfies $A u=0$ as well as the boundary conditions required in Theorem 5.3. We have

$$
\|u\|_{H^{1}(Y)}^{2} \geq\|u\|_{L^{2}(Y)}^{2}=\sum_{\mu_{j} \leq \mu} \int_{\alpha}^{S_{0}-\alpha}\left|\alpha_{j}\right|^{2} \frac{\sinh ^{2}\left(\sqrt{\mu_{j}} s\right)}{\mu_{j}} \mathrm{~d} s \geq \sum_{\mu_{j} \leq \mu}\left|\alpha_{j}\right|^{2} \int_{\alpha}^{S_{0}-\alpha} s^{2} \mathrm{~d} s=C_{S_{0}, \alpha} \sum_{\mu_{j} \leq \mu}\left|\alpha_{j}\right|^{2},
$$

and also

$$
\begin{aligned}
\|u\|_{H^{1}(Z)}^{2} & \leq C \int_{0}^{S_{0}}\left((-\Delta u, u)+\left\|\partial_{s} u\right\|^{2}\right) \mathrm{d} s=C \sum_{\mu_{j} \leq \mu}\left|\alpha_{j}\right|^{2} \int_{0}^{S_{0}}\left(\sinh ^{2}\left(\sqrt{\mu_{j}} s\right)+\cosh ^{2}\left(\sqrt{\mu_{j}} s\right)\right) \mathrm{d} s \\
& \leq C^{\prime} \mathrm{e}^{C^{\prime} \sqrt{\mu}} \sum_{\mu_{j} \leq \mu}\left|\alpha_{j}\right|^{2},
\end{aligned}
$$

using that $\|v\|_{H^{1}(\Omega)}^{2}$ is equivalent to $(-\Delta v, v)$ in $H_{0}^{1}(\Omega)$. Finally, we have $\left\|\partial_{s} u(0, x)\right\|_{L^{2}(\omega)}^{2}=\int_{\omega}\left|\sum_{\mu_{j} \leq \mu} \alpha_{j} \phi_{j}(x)\right|^{2}$ $\mathrm{d} x$, which yields

$$
\sum_{\mu_{j} \leq \mu}\left|\alpha_{j}\right|^{2} \leq C \mathrm{e}^{C \sqrt{\mu}}\left(\sum_{\mu_{j} \leq \mu}\left|\alpha_{j}\right|^{2}\right)^{1-\delta}\left(\int_{\omega}\left|\sum_{\mu_{j} \leq \mu} \alpha_{j} \phi_{j}(x)\right|^{2} \mathrm{~d} x\right)^{\delta}
$$

and the conclusion follows.

On the one hand, in the case $\omega=\Omega$, the result of Theorem 5.4 becomes trivial and the constant $C \mathrm{e}^{C \sqrt{\mu}}$ can be replaced by 1 . On the other hand, it is clear that $K=K(\omega)$ tends to $+\infty$ as the size of $\omega$ goes to zero. An interesting problem would be the precise estimation of $K(\omega)$. Some recent results are available with some lower bonds and uperbounds $[47,57]$.

When $\bar{\omega} \neq \Omega$, the following proposition shows that the power $\frac{1}{2}$ of $\mu$ in $K \mathrm{e}^{K \sqrt{\mu}}$ is optimal (see also [31]).

Proposition 5.5. Let $\omega$ be a non empty open set in $\Omega$ with $\bar{\omega} \neq \Omega$. There exist $C>0$ and $\mu_{0}>0$ such that for all $\mu \geq \mu_{0}$ there exists a sequence $\left(\alpha_{j}\right)_{\mu_{j} \leq \mu}$, such that

$$
\sum_{\mu_{j} \leq \mu}\left|\alpha_{j}\right|^{2} \geq C \mathrm{e}^{C \sqrt{\mu}} \int_{\omega}\left|\sum_{\mu_{j} \leq \mu} \alpha_{j} \phi_{j}(x)\right|^{2} \mathrm{~d} x
$$


Proof We denote by $P_{t}(x, y)$ the heat kernel that we can write $\sum_{j \in \mathbb{N}} \mathrm{e}^{-t \mu_{j}} \phi_{j}(x) \phi_{j}(y)$ for $t>0$; we have $\mathrm{e}^{t \Delta} f(x)=\int P_{t}(x, y) f(y) \mathrm{d} y$. We then write

$$
\left|\sum_{\mu_{j} \leq \mu} \mathrm{e}^{-t \mu_{j}} \phi_{j}(x) \phi_{j}(y)\right| \leq\left|P_{t}(x, y)\right|+\left|\sum_{\mu_{j}>\mu} \mathrm{e}^{-t \mu_{j}} \phi_{j}(x) \phi_{j}(y)\right| .
$$

For $k \in \mathbb{N}$ sufficiently large, Sobolev injections give

$$
\left\|\phi_{j}\right\|_{L^{\infty}} \leq C\left\|\phi_{j}\right\|_{H^{2 k}} \leq C^{\prime}\left\|\Delta^{k} \phi_{j}\right\|_{L^{2}}=C^{\prime} \mu_{j}^{k} .
$$

For all $x, y \in \Omega$ we have $p_{t}(x, y) \leq(4 \pi t)^{-n / 2} \mathrm{e}^{-\frac{|x-y|^{2}}{4 t}}$ by the maximum principle (see Appendix A.7). Let $y_{0}$ be such that $d=\operatorname{dist}\left(y_{0}, \omega\right)>0$. We then have $p_{t}\left(x, y_{0}\right) \leq \mathrm{e}^{-C_{0} / t}$, with $C_{0}>0$, uniformly for $x$ in $\omega$. From (5.5) we thus obtain

$$
\left|\sum_{\mu_{j} \leq \mu} \mathrm{e}^{-t \mu_{j}} \phi_{j}(x) \phi_{j}\left(y_{0}\right)\right| \leq \mathrm{e}^{-C_{0} / t}+C \sum_{\mu_{j}>\mu} \mathrm{e}^{-t \mu_{j}} \mu_{j}^{2 k}, \quad x \in \omega .
$$

We choose $\alpha_{j}=\mathrm{e}^{-t \mu_{j}} \phi_{j}\left(y_{0}\right)$ and we take $t=1 / \sqrt{\mu}$. We have

$$
\left|\sum_{\mu_{j} \leq \mu} \alpha_{j} \phi_{j}(x)\right| \leq C \mathrm{e}^{-C_{0} \sqrt{\mu}}+C \sum_{\mu_{j}>\mu} \mathrm{e}^{-t \mu_{j}} \mu_{j}^{2 k}, \quad x \in \omega .
$$

To estimate the second term we introduce $J_{\mu}=\left\{l ; \mu_{l} \leq \mu\right\}$. The Weyl asymptotics (see e.g. [1]) yields $\# J_{\mu} \leq C \mu^{n / 2}$. Then, for $\mu>1$ large, we write

$$
\begin{aligned}
\sum_{\mu_{j}>\mu} \mathrm{e}^{-t \mu_{j}} \mu_{j}^{2 k} & =\sum_{\substack{N \in \mathbb{N} \\
N<\mu_{j}-\mu \leq N+1}} \mathrm{e}^{-t \mu_{j}} \mu_{j}^{2 k} \leq \sum_{\substack{N \in \mathbb{N} \\
N<\mu_{j}-\mu \leq N+1}} \mathrm{e}^{-t(\mu+N)}(\mu+N+1)^{2 k} \leq \sum_{N \in \mathbb{N}} \# J_{\mu+N+1} \mathrm{e}^{-t(\mu+N)}(\mu+N+1)^{2 k} \\
& \leq C \sum_{N \in \mathbb{N}} \mathrm{e}^{-t(\mu+N)}(\mu+N+1)^{2 k+n / 2} \leq C \int_{\mu-1}^{\infty} \mathrm{e}^{-t x}(x+1)^{2 k+n / 2} \mathrm{~d} x=C \mathrm{e}^{t} \int_{\mu}^{\infty} \mathrm{e}^{-t x} x^{2 k+n / 2} \mathrm{~d} x .
\end{aligned}
$$

In fact, with $t=1 / \sqrt{\mu}$, the function $\mathrm{e}^{-t x} x^{2 k+n / 2}$ decreases in $[\mu,+\infty)$ for $\mu$ large. We set $l=2 k+n / 2$. The change of variable $y=t(x-\mu)$ then yields

$$
\begin{aligned}
\int_{\mu}^{\infty} \mathrm{e}^{-t x} x^{l} \mathrm{~d} x & =t^{-1-l} \mathrm{e}^{-t \mu} \int_{0}^{\infty} \mathrm{e}^{-y}(\mu t+y)^{l} \mathrm{~d} y=\mu^{\frac{l+1}{2}} \mathrm{e}^{-\sqrt{\mu}} \int_{0}^{\infty} \mathrm{e}^{-y}(\sqrt{\mu}+y)^{l} \mathrm{~d} y \\
& \leq \mu^{l+\frac{1}{2}} \mathrm{e}^{-\sqrt{\mu}} \int_{0}^{\infty} \mathrm{e}^{-y}(1+y)^{l} \mathrm{~d} y=C \mu^{l+\frac{1}{2}} \mathrm{e}^{-\sqrt{\mu}},
\end{aligned}
$$

since $t=1 / \sqrt{\mu}$. For $\mu>1$ large, we have thus obtained $\left|\sum_{\mu_{j} \leq \mu} \alpha_{j} \phi_{j}(x)\right| \leq C \mathrm{e}^{-C \sqrt{\mu}}$, which yields

$$
\int_{\omega}\left|\sum_{\mu_{j} \leq \mu} \alpha_{j} \phi_{j}(x)\right|^{2} \mathrm{~d} x \leq C|\omega| \mathrm{e}^{-C \sqrt{\mu}}
$$

We now conclude by proving $\sum_{\mu_{j} \leq \mu}\left|\alpha_{j}\right|^{2} \geq C \mu^{n / 4} \geq 1$, for $\mu$ sufficiently large with the choice of coefficients $\alpha_{j}, j \in \mathbb{N}$, we have made above. In fact we find

$$
\sum_{\mu_{j} \leq \mu}\left|\alpha_{j}\right|^{2}=\sum_{\mu_{j} \leq \mu} \mathrm{e}^{-2 t \mu_{j}}\left|\phi_{j}\left(y_{0}\right)\right|^{2}=P_{2 t}\left(y_{0}, y_{0}\right)-\sum_{\mu_{j}>\mu} \mathrm{e}^{-2 t \mu_{j}}\left|\phi_{j}\left(y_{0}\right)\right|^{2} .
$$


As here $t=1 / \sqrt{\mu}$ is small, Lemma A.5 (see Appendix A.7) gives $P_{2 t}\left(y_{0}, y_{0}\right) \geq C(2 t)^{-n / 2}=C^{\prime} \mu^{n / 4}$. Finally, using Sobolev inequalities as above we obtain the following estimate $\sum_{\mu_{j}>\mu} \mathrm{e}^{-2 t \mu_{j}}\left|\phi_{j}\left(y_{0}\right)\right|^{2} \leq C \sum_{\mu_{j}>\mu} \mathrm{e}^{-2 t \mu_{j}} \mu_{j}^{2 k} \leq$ $C^{\prime} \mathrm{e}^{-C^{\prime} \sqrt{\mu}}$

The spectral inequality of Theorem 5.4 also leads to the following unique continuation result for series of eigenfunctions.

Proposition 5.6. Let $\omega \subset \Omega$ be open and $\varepsilon>0$. Then for all functions $u=\sum_{j \in \mathbb{N}^{*}} \alpha_{j} \phi_{j}$ with the complex coefficients $\alpha_{j}$ satisfying $\left|\alpha_{j}\right| \leq \mathrm{e}^{-\varepsilon \sqrt{\mu_{j}}}, j \in \mathbb{N}^{*}$, we have $u=0$ if $\left.u\right|_{\omega}=0$.

This result yields an analogy between the series $\sum_{j \in \mathbb{N}^{*}} \alpha_{j} \phi_{j}$ and analytic functions, when the coefficients $\alpha_{j}$ satisfy the asymptotics $\left|\alpha_{j}\right| \leq \mathrm{e}^{-\varepsilon \sqrt{\mu_{j}}}$.

Proof For $0 \leq s<\varepsilon$ we set $v(s, x)=\sum_{j \in \mathbb{N}^{*}} \alpha_{j} \frac{\sinh \left(\sqrt{\mu_{j}} s\right)}{\sqrt{\mu_{j}}} \phi_{j}(x)$. The asymptotic behavior we have assumed for the coefficients $\alpha_{j}$ yields $v \in \mathscr{C}^{2}\left((0, \varepsilon), H^{2}(\Omega)\right)$. We then apply the interpolation inequality of Theorem 5.3 taking $Y=\left(\alpha, S_{0}-\alpha\right) \times \Omega$ with $0<\alpha<S_{0}-\alpha<S_{0}<\varepsilon$. Since $v$ satisfies the proper boundary conditions and since $A v=0$ and $\left.\partial_{s} v\right|_{\{0\} \times \omega}=\left.u\right|_{\omega}=0$, this yields $\|v\|_{H^{1}(Y)}=0$. For almost every $s \in\left(\alpha, S_{0}-\alpha\right)$ we thus have $x \mapsto \sum_{j \in \mathbb{N}^{*}} \alpha_{j} \frac{\sinh \left(\sqrt{\mu_{j}} s\right)}{\sqrt{\mu_{j}}} \phi_{j}(x)=0$ in $L^{2}(\Omega)$. The orthogonality of the eigenfunctions gives $\alpha_{j}=0$ for all $j \in \mathbb{N}^{*}$.

\section{Control of the heat equation}

We shall now construct a control function for the heat equation in the time interval $(0, T)$ for an initial condition $y_{0}$ in $L^{2}(\Omega)$,

$$
\begin{cases}\partial_{t} y-\Delta y=1_{\omega} v & \text { in } Q=(0, T) \times \Omega \\ y=0 & \text { on } \Sigma=(0, T) \times \partial \Omega \\ y(0)=y_{0} & \text { in } \Omega .\end{cases}
$$

The function $v$ is the control. The goal is to drive the solution $y$ to zero at time $T>0$, yet only acting in the sub-domain $\omega$.

We start with a partial control result. Next, in Section 6.2 , the control $v$ will be built as a sequence of active and passive controls. The passive mode allows to take advantage of the natural parabolic exponential decay of the $L^{2}$ norm of the solution. A similar approach, on the observability side, can be found in $[47,57]$.

\subsection{Observability and partial control}

For $j \in \mathbb{N}$, we define the finite dimensional space $E_{j}=\operatorname{span}\left\{\phi_{k} ; \mu_{k} \leq 2^{2 j}\right\}$ and the following null controllability problem

$$
\begin{cases}\partial_{t} y-\Delta y=\Pi_{E_{j}}\left(1_{\omega} v\right) & \text { in }(0, \mathcal{T}) \times \Omega \\ y=0 & \text { on }(0, \mathcal{T}) \times \partial \Omega \\ y(0)=y_{0} \in E_{j} & \text { in } \Omega\end{cases}
$$

with $\mathcal{T}>0$ and where $\Pi_{E_{j}}$ denotes the orthogonal projection onto $E_{j}$ in $L^{2}(\Omega)$. We estimate the so-called control cost, i.e., the $L^{2}$ norm of the control function $v$ that gives $y(\mathcal{T})=0$.

Lemma 6.1. There exists a control function $v$ that drives the solution of system (6.2) to zero at time $\mathcal{T}$ and $\|v\|_{L^{2}((0, T) \times \omega)} \leq C \mathcal{T}^{-\frac{1}{2}} \mathrm{e}^{C 2^{j}}\left\|y_{0}\right\|_{L^{2}(\Omega)}$. 
For $a \geq 0$, when we consider the time interval $[a, a+\mathcal{T}]$, we shall denote by $V_{j}\left(y_{0}, a, \mathcal{T}\right)$ such a control satisfying $\left\|V_{j}\left(y_{0}, a, \mathcal{T}\right)\right\|_{L^{2}((a, a+\mathcal{T}) \times \Omega)} \leq C \mathcal{T}^{-\frac{1}{2}} \mathrm{e}^{C 2^{j}}\left\|y_{0}\right\|_{L^{2}(\Omega)}$.

Proof The adjoint system of (6.2) is

$$
\begin{cases}-\partial_{t} q-\Delta q=0 & \text { in }(0, \mathcal{T}) \times \Omega \\ q=0 & \text { on }(0, \mathcal{T}) \times \partial \Omega \\ q(\mathcal{T})=q_{f} \in E_{j} . & \end{cases}
$$

If we write $q(0)=\sum_{\mu_{k} \leq 2^{2 j}} b_{k} \phi_{k}$ then $q(t)=\sum_{\mu_{k} \leq 2^{2 j}} \alpha_{k}(t) \phi_{k}$ with $\alpha_{k}(t)=b_{k} \mathrm{e}^{\mu_{k} t}$ and we thus have

$$
\begin{aligned}
\mathcal{T}\|q(0)\|_{L^{2}(\Omega)}^{2} & \leq \int_{0}^{\mathcal{T}}\|q(t)\|_{L^{2}(\Omega)}^{2} \mathrm{~d} t=\int_{0}^{\mathcal{T}} \int_{\Omega}\left|\sum_{\mu_{k} \leq 2^{2 j}} \alpha_{k}(t) \phi_{k}\right|^{2} \mathrm{~d} t \mathrm{~d} x \\
& \leq C \mathrm{e}^{C 2^{j}} \int_{0}^{\mathcal{T}} \int_{\omega}\left|\sum_{\mu_{k} \leq 2^{2 j}} \alpha_{k}(t) \phi_{k}\right|^{2} \mathrm{~d} t \mathrm{~d} x=C \mathrm{e}^{C 2^{j}} \int_{0}^{\mathcal{T}} \int_{\omega}|q(t)|^{2} \mathrm{~d} t \mathrm{~d} x,
\end{aligned}
$$

because of the parabolic decay and from the spectral inequality of Theorem 5.4. This observability inequality yields the expected estimate of the cost of the control.

\subsection{Construction of the control function}

We split the time interval $[0, T]$ into sub-intervals, $[0, T]=\bigcup_{j \in \mathbb{N}}\left[a_{j}, a_{j+1}\right]$, with $a_{0}=0, a_{j+1}=a_{j}+2 T_{j}$, for $j \in \mathbb{N}$ and $T_{j}=K 2^{-j \rho}$ with $\rho \in(0,1)$ and the constant $K$ chosen such that $2 \sum_{j=0}^{\infty} T_{j}=T$. We now define the control function $v$ according to the strategy exposed above:

$$
\begin{aligned}
& \text { if } t \in\left(a_{j}, a_{j}+T_{j}\right], \quad v(t, x)=V_{j}\left(\Pi_{E_{j}} y\left(a_{j}, .\right), a_{j}, T_{j}\right) \\
& \text { and } y(t, .)=S\left(t-a_{j}\right) y\left(a_{j}, .\right)+\int_{a_{j}}^{t} S(t-s) v(s, .) \mathrm{d} s, \\
& \text { if } t \in\left(a_{j}+T_{j}, a_{j+1}\right], \quad v(t, x)=0 \quad \text { and } y(t, .)=S\left(t-a_{j}-T_{j}\right) y\left(a_{j}+T_{j}, .\right),
\end{aligned}
$$

where $S(t)$ denotes the heat semi-group $S(t)=\mathrm{e}^{t \Delta}$. In particular, $\|S(t)\|_{\left(L^{2}, L^{2}\right)} \leq 1$.

The choice of the control $v$ in the time interval $\left[a_{j}, a_{j}+T_{j}\right], j \in \mathbb{N}$, yields

$$
\left\|y\left(a_{j}+T_{j}, .\right)\right\|_{L^{2}(\Omega)} \leq\left(1+C \mathrm{e}^{C 2^{j}}\right)\left\|y\left(a_{j}, .\right)\right\|_{L^{2}(\Omega)}, \quad \text { and } \quad \Pi_{E_{j}} y\left(a_{j}+T_{j}, .\right)=0 .
$$

During the passive mode, $t \in\left[a_{j}+T_{j}, a_{j+1}\right]$, the solution is subject to an exponential decay

$$
\left\|y\left(a_{j+1}, .\right)\right\|_{L^{2}(\Omega)} \leq \mathrm{e}^{-2^{2 j} T_{j}}\left\|y\left(a_{j}+T_{j}, .\right)\right\|_{L^{2}(\Omega)} .
$$

We thus obtain $\left\|y\left(a_{j+1}, .\right)\right\|_{L^{2}(\Omega)} \leq \mathrm{e}^{C 2^{j}-2^{2 j} T_{j}}\left\|y\left(a_{j}, .\right)\right\|_{L^{2}(\Omega)}$, and hence we have

$$
\left\|y\left(a_{j+1}, .\right)\right\|_{L^{2}(\Omega)} \leq \mathrm{e}^{\sum_{k=0}^{j} C 2^{k}-2^{2 k} T_{k}}\left\|y_{0}\right\|_{L^{2}(\Omega)}, \quad j \in \mathbb{N} .
$$

We have $2^{2 k} T_{k}=K 2^{k(2-\rho)}$. We observe that $2-\rho>1$ which yields $\lim _{j \rightarrow \infty} \sum_{k=0}^{j}\left(C 2^{k}-K 2^{k(2-\rho)}\right)=-\infty$. For a certain constant $C>0$ we have

$$
\left\|y\left(a_{j+1}, .\right)\right\|_{L^{2}(\Omega)} \leq C \mathrm{e}^{-C 2^{j(2-\rho)}}\left\|y_{0}\right\|_{L^{2}(\Omega)}, \quad j \in \mathbb{N} .
$$


We conclude that $\lim _{j \rightarrow \infty}\left\|y\left(a_{j}, .\right)\right\|_{L^{2}(\Omega)}=0$, i.e. $y(T,)=$.0 since $y(t,$.$) is continuous with values in L^{2}(\Omega)$ since the r.h.s. of $(6.1)$ is in $L^{2}(Q)$ by construction as we shall now see.

We have $\|v\|_{L^{2}(Q)}^{2}=\sum_{j \geq 0}\|v\|_{L^{2}\left(\left(a_{j}, a_{j}+T_{j}\right) \times \Omega\right)}^{2}$. From the cost of the control given in Lemma 6.1 and (6.3) we deduce

$$
\|v\|_{L^{2}(Q)}^{2} \leq\left(C T_{0}^{-1} \mathrm{e}^{2 C}+\sum_{j \geq 1} C T_{j}^{-1} \mathrm{e}^{C 2^{j}} \mathrm{e}^{-C 2^{(j-1)(2-\rho)}}\right)\left\|y_{0}\right\|_{L^{2}(\Omega)}^{2} .
$$

As $2-\rho>1$ and $T_{j}=K 2^{-j \rho}$, arguing as above we obtain $\|v\|_{L^{2}(Q)} \leq C_{T}\left\|y_{0}\right\|_{L^{2}(\Omega)}$ with $C_{T}<\infty$. We have thus obtain the following null controllability result.

Theorem 6.2 (null controllability [39]). For all $T>0$, there exists $C_{T}>0$ such that for all initial conditions $y_{0} \in L^{2}(\Omega)$, there exists $v \in L^{2}(Q)$, with $\|v\|_{L^{2}(Q)} \leq C_{T}\left\|y_{0}\right\|_{L^{2}(\Omega)}$, such that the solution to system (6.1) satisfies $y(T)=0$.

Corollary 6.3 (observability). There exists $C_{T}>0$ such that the solution $y \in \mathscr{C}\left([0, T], L^{2}(\Omega)\right)$ of the adjoint system

$$
\begin{cases}-\partial_{t} q-\Delta q=0 & \text { in } Q \\ q=0 & \text { on } \Sigma, \\ q(T)=q_{T} & \text { in } \Omega,\end{cases}
$$

satisfies the following observability inequality $\|q(0)\|_{L^{2}(\Omega)}^{2} \leq C_{T}^{2} \int_{0}^{T} \int_{\omega}|q(t)|^{2} \mathrm{~d} t \mathrm{~d} x$.

\section{Carleman estimates for Parabolic operators}

Here we shall prove Carleman estimates for parabolic operators, typically $P=\partial_{t}+A$ with $A=-\Delta$. As in the previous sections $\Omega$ is a bounded open set in $\mathbb{R}^{n}$. We set $Q=(0, T) \times \Omega$. We start by proving local (in space) estimates, away from the boundary $\partial \Omega$.

\subsection{Local estimates}

We set $\theta(t)=t(T-t)$ and $h=\varepsilon \theta(t)$. The parameter $\varepsilon$ will be small, $0<\varepsilon \leq \varepsilon_{0}\langle\langle 1$. For a weight function $\varphi(x)$ we define $P_{\varphi}=h^{2} \mathrm{e}^{\varphi / h} P \mathrm{e}^{-\varphi / h}$. The semi-classical parameter $h$ depends on the time variable $t$ here, and moreover vanishes for $t=0$ and $t=T$.

We have

$$
P_{\varphi}=h^{2} \partial_{t}+\varepsilon \varphi(x) \theta^{\prime}(t)-h^{2} \Delta+2 h\left\langle\varphi^{\prime}, \nabla_{x}\right\rangle-\left|\varphi^{\prime}\right|^{2}+h \Delta \varphi .
$$

We define the following symmetric operators

$$
Q_{2}=\left(P_{\varphi}+P_{\varphi}^{*}\right) / 2, \quad Q_{1}=\left(P_{\varphi}-P_{\varphi}^{*}\right) /(2 i),
$$

which gives

$$
\begin{aligned}
& Q_{2}=-\varepsilon h \theta^{\prime}(t)+\varepsilon \varphi(x) \theta^{\prime}(t)-h^{2} \Delta-\left|\varphi^{\prime}\right|^{2}, \\
& Q_{1}=\frac{h^{2}}{i} \partial_{t}+\frac{\varepsilon h}{i} \theta^{\prime}(t)+\frac{h}{i} \Delta \varphi+\frac{2 h}{i}\left\langle\varphi^{\prime}, \nabla_{x}\right\rangle,
\end{aligned}
$$

with respective principal symbols

$$
q_{2}=\varepsilon \varphi(x) \theta^{\prime}(t)+|\xi|^{2}-\left|\varphi^{\prime}\right|^{2}, \quad q_{1}=h \tau+2\left\langle\varphi^{\prime}, \xi\right\rangle .
$$

We have $p_{\varphi}=q_{2}+i q_{1}$ and $P_{\varphi}=Q_{2}+i Q_{1}$.

We choose the weight function $\varphi$ according to the following assumption. 
Assumption 7.1. Let $V$ be an open subset of $\Omega$. The weight function $\varphi$ satisfies

$$
\begin{aligned}
& \varphi(x)<0, \quad\left|\varphi^{\prime}(x)\right| \neq 0, x \in \bar{V}, \\
& \left.q_{2}\right|_{\varepsilon=0}=0 \Rightarrow\left\{\left.q_{2}\right|_{\varepsilon=0},\left.q_{1}\right|_{\varepsilon=0}\right\}>0, \quad x \in \bar{V}, \xi \in \mathbb{R}^{n},
\end{aligned}
$$

These conditions, stronger than those we presented in the elliptic case, were introduced in [38]. We shall see below that they are sufficient and also necessary for a Carleman estimate to hold. They only involve the spatial variables, $x$ and $\xi$, and can be fulfilled by choosing $\varphi$ of the form

$$
\varphi(x)=\mathrm{e}^{\lambda \psi(x)}-\mathrm{e}^{\lambda L}, \quad \text { with } L>\|\psi\|_{\infty}, \quad\left|\psi^{\prime}(x)\right| \neq 0, x \in \bar{V},
$$

and letting the positive parameter $\lambda$ be sufficiently large (see Lemma A.1 in Sect. A.2).

With this assumption we can prove the following lemma (see Appendix A.8 for a proof).

Lemma 7.2. There exist $C>0, \mu_{1}>0$ and $\delta_{1}>0$, such that for $\mu \geq \mu_{1}$ and $0 \leq \varepsilon T \leq \delta_{1}$ we have

$$
\mu q_{2}^{2}-2 \varepsilon \theta^{\prime}|\xi|^{2}+\left\{q_{2}, b\right\} \geq C\langle\xi\rangle^{4}, \quad x \in \bar{V}, \quad \xi \in \mathbb{R}^{n},
$$

where $b:=2\left\langle\varphi^{\prime}, \xi\right\rangle$.

We can now prove the following Carleman estimate, that is local in space and global in time, for the parabolic operator $P$.

Theorem 7.3 (local Carleman estimate away from the boundary). Let $K$ be a compact set of $\Omega$ and $V$ an open subset of $\Omega$ that is a neighborhood of $K$. Let $\varphi$ be a weight function that satisfies Assumption 7.1 in $\bar{V}$. Then there exist $C>0$ and $\delta_{2}>0$ such that

$$
\left\|h^{\frac{1}{2}} \mathrm{e}^{\varphi / h} u\right\|_{L^{2}(Q)}^{2}+\left\|h^{3 / 2} \mathrm{e}^{\varphi / h} \nabla_{x} u\right\|_{L^{2}(Q)}^{2} \leq C\left\|h^{2} \mathrm{e}^{\varphi / h} P u\right\|_{L^{2}(Q)}^{2}
$$

for $u \in \mathscr{C}^{\infty}([0, T] \times \Omega)$, with $u(t) \in \mathscr{C}_{c}^{\infty}(K)$ for all $t \in[0, T]$, and $0<\left(T+T^{2}\right) \varepsilon \leq \delta_{2}$.

Proof We introduce $v=\mathrm{e}^{\varphi / h} u$. We observe that $v$, along with all its time derivatives, vanishes at time $t=0$ and $t=T$, since $\varphi \leq-C<0$ in $K$. We have $P_{\varphi} v=h^{2} \mathrm{e}^{\varphi / h} P u=g$ and we write, similarly to (3.2),

$$
\|g\|_{L^{2}(Q)}^{2}=\left\|Q_{1} v\right\|_{L^{2}(Q)}^{2}+\left\|Q_{2} v\right\|_{L^{2}(Q)}^{2}+i\left(\left[Q_{2}, Q_{1}\right] v, v\right)_{L^{2}(Q)},
$$

which yields, with $B=Q_{1}-\frac{h^{2}}{i} \partial_{t}-\frac{\varepsilon h}{i} \theta^{\prime}(t)=\frac{h}{i} \Delta \varphi+\frac{2 h}{i}\left\langle\varphi^{\prime}, \nabla_{x}\right\rangle$,

$$
\begin{aligned}
\|g\|_{L^{2}(Q)}^{2} & =\left\|Q_{1} v\right\|_{L^{2}(Q)}^{2}+\left\|Q_{2} v\right\|_{L^{2}(Q)}^{2}+\left(\left(-h^{2}\left(\partial_{t} Q_{2}\right)+i\left[Q_{2}, B\right]\right) v, v\right)_{L^{2}(Q)} \\
& \geq\left(\left(h \mu Q_{2}^{2}-h^{2}\left(\partial_{t} Q_{2}\right)+i\left[Q_{2}, B\right]\right) v, v\right)_{L^{2}(Q)}=\left(h\left(\mu Q_{2}^{2}-h\left(\partial_{t} Q_{2}\right)+\frac{i}{h}\left[Q_{2}, B\right]\right) v, v\right)_{L^{2}(Q)}
\end{aligned}
$$

for $\mu>0$ and $0<h<1 / \mu$. We note that $h\left(\partial_{t} Q_{2}\right)=-\varepsilon h^{2} \theta^{\prime \prime}-\varepsilon^{2} h\left(\theta^{\prime}\right)^{2}+\varepsilon h \theta^{\prime \prime} \varphi-2 \varepsilon \theta^{\prime} h^{2} \Delta$. The principal symbol of $\mu Q_{2}^{2}-h\left(\partial_{t} Q_{2}\right)+\frac{i}{h}\left[Q_{2}, B\right]$ is $\mu q_{2}^{2}-2 \varepsilon \theta^{\prime}|\xi|^{2}+\left\{q_{2}, b\right\}$. We choose $\mu_{1}>0$ and $\delta_{1}>0$ according to Lemma 7.2 and we take $0<\varepsilon T \leq \delta_{1}$. The Gårding inequality is uniform with respect to the semi-classical parameter $h$, once taken sufficiently small (i.e., by taking $0<\varepsilon \theta<\varepsilon T^{2} / 4 \leq \delta_{1}^{\prime}$ for $\delta_{1}^{\prime}$ sufficiently small, for instance), and we obtain

$$
\left(\left(\mu Q_{2}^{2}-h\left(\partial_{t} Q_{2}\right)+\frac{i}{h}\left[Q_{2}, B\right]\right) v(t), v(t)\right)_{L^{2}(\Omega)} \geq C\|v(t)\|_{2}^{2}, \quad \forall t \in[0, T]
$$

for $\mu \geq \mu_{1}$ and $0<\left(T+T^{2}\right) \varepsilon \leq \delta_{2}=\min \left(\delta_{1}, 4 \delta_{1}^{\prime}\right)$, and it follows that $\|g\|_{L^{2}(Q)}^{2} \geq C \int_{0}^{T} h\|v\|_{2}^{2} \mathrm{~d} t$. We then obtain the sought local Carleman estimate by arguing as in the end of the proof of Theorem 3.5. 
Remark 7.4. In the proof of the previous theorem, we note the importance of only relying on the non-negative term $\left\|Q_{2} v\right\|_{L^{2}(Q)}^{2}$ since the other square term $\left\|Q_{1} v\right\|_{L^{2}(Q)}^{2}$ involves a time derivative of $v$, and cannot be used in the Gårding inequality (7.2) at fixed $t$. If we chose to use a Gårding inequality with respect to all variables $(t, x)$, we could then use both square terms $\left\|Q_{2} v\right\|_{L^{2}(Q)}^{2}$ and $\left\|Q_{1} v\right\|_{L^{2}(Q)}^{2}$. This is the scheme of the proof that we shall follow to prove an estimate at the boundary below.

The following proposition shows the necessity of Assumption 7.1. See Appendix A.9 for a proof.

Proposition 7.5. Let $V$ be an open subset of $\Omega, \varphi(x)$ be defined on $\bar{V}$, and $\delta>0$ and $C>0$ be such that for a certain $\alpha \leq \frac{1}{2}$ we have

$$
\left\|h^{\alpha} \mathrm{e}^{\varphi / h} u\right\|_{L^{2}(Q)} \leq C\left\|h^{2} \mathrm{e}^{\varphi / h} P u\right\|_{L^{2}(Q)}
$$

for all $u \in \mathscr{C}^{\infty}([0, T] \times \Omega)$, with $u(t) \in \mathscr{C}_{c}^{\infty}(V)$ for all $t \in[0, T]$, and $0<\left(T+T^{2}\right) \varepsilon \leq \delta$. Then $\alpha=\frac{1}{2}$ and the weight function $\varphi$ satisfies

$$
\begin{aligned}
& \left|\varphi^{\prime}(x)\right| \neq 0, \quad x \in \bar{V}, \\
& \left.q_{2}\right|_{\varepsilon=0}=0 \Rightarrow\left\{\left.q_{2}\right|_{\varepsilon=0},\left.q_{1}\right|_{\varepsilon=0}\right\}>0, \quad x \in \bar{V}, \xi \in \mathbb{R}^{n} .
\end{aligned}
$$

\subsection{Estimate at the boundary}

If we place ourselves in the neighborhood of the boundary we have the following result.

Theorem 7.6 (Carleman estimate at the boundary). Let $x_{0} \in \partial \Omega$ and $K$ be a compact set of $\bar{\Omega}, x_{0} \in K$, and $V$ an open subset of $\bar{\Omega}$ that is a neighborhood of $K$ in $\bar{\Omega}$, with $K$ and $V$ chosen sufficiently small. Let $\varphi$ be a weight function that satisfies Assumption 7.1 in $\bar{V}$, and $\left.\partial_{n} \varphi\right|_{\partial \Omega \cap V}<0$, where $n$ is the outward pointing unit normal to $\Omega$. Then there exist $C>0$ and $\delta_{3}>0$ such that

$$
\left\|h^{\frac{1}{2}} \mathrm{e}^{\varphi / h} u\right\|_{L^{2}(Q)}^{2}+\left\|h^{3 / 2} \mathrm{e}^{\varphi / h} \nabla_{x} u\right\|_{L^{2}(Q)}^{2} \leq C\left\|h^{2} \mathrm{e}^{\varphi / h} P u\right\|_{L^{2}(Q)}^{2}
$$

for $0<\left(T+T^{2}\right) \varepsilon \leq \delta_{3}, h=\varepsilon t(T-t)$ and $u \in \mathscr{C}^{\infty}([0, T] \times \bar{\Omega})$, with $\operatorname{supp}(u(t)) \subset K$ for all $t \in[0, T]$, and $\left.u\right|_{(0, T) \times(\partial \Omega \cap V)}=0$.

The proof of this estimate is more technical than that of Theorem 7.3. We have placed it in Appendix A.10. The idea of the proof is to use the Gårding inequality in the tangential directions, including the time direction. The original proof for this estimate is available in [22]. However, following the approach of [22] does not put forward the sufficiency of Assumption 7.1.

\subsection{Global estimate}

We now focus our attention on global Carleman estimates. We proceed by patching together the local estimates we have presented here, in the interior and at the boundary. The global aspect of the estimate will impose an "observation" term over $(0, T) \times \omega$, with $\omega \Subset \Omega$ in the r.h.s. of the estimate.

To patch these local estimates together we choose a global weight function that can be used to derive each of these local estimates by satisfying the following requirements.

Assumption 7.7. Let $\omega_{0} \Subset \omega \Subset \Omega$. The weight function $\varphi$ satisfies

$$
\begin{aligned}
& \left.\varphi\right|_{\partial \Omega}=\text { Cst, }\left.\quad \partial_{n} \varphi\right|_{\partial \Omega}<0, \quad \sup _{x \in \bar{\Omega}} \varphi(x)<0, \quad\left|\varphi^{\prime}(x)\right| \neq 0, x \in \Omega \backslash \omega_{0}, \\
& \left.q_{2}\right|_{\varepsilon=0}=0 \Rightarrow\left\{\left.q_{2}\right|_{\varepsilon=0},\left.q_{1}\right|_{\varepsilon=0}\right\}>0, \quad x \in \Omega \backslash \omega_{0} .
\end{aligned}
$$


Such conditions can be fulfilled by taking $\varphi$ of the form

$$
\begin{aligned}
& \varphi(x)=\mathrm{e}^{\lambda \psi(x)}-\mathrm{e}^{\lambda K}, \quad \text { with } K>\|\psi\|_{\infty}, \quad\left|\psi^{\prime}(x)\right| \neq 0, x \in \Omega \backslash \omega_{0}, \quad \text { and } \\
& \left.\psi\right|_{\partial \Omega}=0,\left.\quad \partial_{n} \psi\right|_{\partial \Omega}<0, \quad \psi(x)>0, x \in \Omega,
\end{aligned}
$$

and by taking the positive parameter $\lambda$ sufficiently large. For the construction of such a function $\psi$ we refer to [22], Lemma 1.1. The construction makes use of Morse functions and the associated approximation theorem [3].

Theorem 7.8 (global Carleman estimate). Let $\varphi$ be a function that satisfies Assumption 7.7. Then there exist $\delta_{4}>0$ and $C \geq 0$ such that

$$
\left\|h^{\frac{1}{2}} \mathrm{e}^{\varphi / h} u\right\|_{L^{2}(Q)}^{2}+\left\|h^{3 / 2} \mathrm{e}^{\varphi / h} \nabla_{x} u\right\|_{L^{2}(Q)}^{2} \leq C\left(\left\|h^{2} \mathrm{e}^{\varphi / h} P u\right\|_{L^{2}(Q)}^{2}+\left\|h^{\frac{1}{2}} \mathrm{e}^{\varphi / h} u\right\|_{L^{2}((0, T) \times \omega)}^{2}\right),
$$

for $0<\left(T+T^{2}\right) \varepsilon \leq \delta_{4}, h=\varepsilon t(T-t)$ and $u \in \mathscr{C}^{\infty}([0, T] \times \bar{\Omega})$ such that $\left.u\right|_{(0, T) \times \partial \Omega}=0$.

Proof Let $\omega_{1}$ be such that $\omega_{0} \Subset \omega_{1} \Subset \omega$. For all $x \in \bar{\Omega} \backslash \omega_{1}$, there exist an open subset $V_{x}$ of $\bar{\Omega}$, with $x \in V_{x} \subset \bar{\Omega} \backslash \omega_{0}$ for which the local Carleman estimate, in the interior or at the boundary, holds with the weight function $\varphi$, for smooth functions with support in the compact $K_{x}=\overline{V_{x}}$.

From the covering of $\bar{\Omega} \backslash \omega_{1}$ by the open sets $V_{x}, x \in \bar{\Omega} \backslash \omega_{1}$ we can extract a finite covering $\left(V_{i}\right)_{i \in \mathcal{I}}$, such that for all $i \in \mathcal{I}$ the Carleman estimate in $V_{i}$ holds for $h<h_{i}, C=C_{i}>0$ and $\operatorname{supp}(u) \subset K_{i}=\bar{V}_{i}$.

Let $\left(\chi_{i}\right)_{i \in \mathcal{I}}$ be a partition of unity subordinated to the covering $V_{i}, i \in \mathcal{I}$, [28,58], i.e.,

$$
\chi_{i} \in \mathscr{C}^{\infty}(\bar{\Omega}), \quad \operatorname{supp}\left(\chi_{i}\right) \subset K_{i}=\overline{V_{i}}, \quad 0 \leq \chi_{i} \leq 1, \quad i \in \mathcal{I}, \quad \text { and } \quad \sum_{i \in \mathcal{I}} \chi_{i}=1 \text { in } \Omega \backslash \omega_{1} .
$$

Note that we have $\operatorname{supp}\left(\chi_{i}\right) \cap \omega_{0}=\emptyset$. For all $i \in \mathcal{I}$, we set $u_{i}=\chi_{i} u$. Then for each $u_{i}$ we have a local Carleman estimate. We now observe that we have

$$
P u_{i}=P\left(\chi_{i} u\right)=\chi_{i} P u+\left[P, \chi_{i}\right] u=\chi_{i} P u-\left[\Delta, \chi_{i}\right] u,
$$

where the commutator is a first-order differential operator in $x$. For all $i \in \mathcal{I}$, we thus obtain

$$
\begin{aligned}
\left\|h^{2} \mathrm{e}^{\varphi / h} P u_{i}\right\|_{L^{2}(Q)}^{2} & \leq C\left\|h^{2} \mathrm{e}^{\varphi / h} P u\right\|_{L^{2}(Q)}^{2}+C\left\|h^{2} \mathrm{e}^{\varphi / h} u\right\|_{L^{2}(Q)}^{2}+C\left\|h^{2} \mathrm{e}^{\varphi / h} \nabla_{x} u\right\|_{L^{2}(Q)}^{2} \\
& \leq C\left\|h^{2} \mathrm{e}^{\varphi / h} P u\right\|_{L^{2}(Q)}^{2}+C\left(\varepsilon T^{2}\right)^{3}\left\|h^{\frac{1}{2}} \mathrm{e}^{\varphi / h} u\right\|_{L^{2}(Q)}^{2}+C \varepsilon T^{2}\left\|h^{3 / 2} \mathrm{e}^{\varphi / h} \nabla_{x} u\right\|_{L^{2}(Q)}^{2} .
\end{aligned}
$$

We note that we have

$$
\begin{aligned}
\left\|h^{\frac{1}{2}} \mathrm{e}^{\varphi / h} u\right\|_{L^{2}(Q)}^{2}+\left\|h^{3 / 2} \mathrm{e}^{\varphi / h} \nabla_{x} u\right\|_{L^{2}(Q)}^{2} \leq & C \sum_{i \in \mathcal{I}}\left(\left\|h^{\frac{1}{2}} \mathrm{e}^{\varphi / h} u_{i}\right\|_{L^{2}(Q)}^{2}+\left\|h^{3 / 2} \mathrm{e}^{\varphi / h} \nabla_{x} u_{i}\right\|_{L^{2}(Q)}^{2}\right) \\
& +C\left\|h^{\frac{1}{2}} \mathrm{e}^{\varphi / h} u\right\|_{L^{2}\left((0, T) \times \omega_{1}\right)}^{2}+C\left\|h^{3 / 2} \mathrm{e}^{\varphi / h} \nabla_{x} u\right\|_{L^{2}\left((0, T) \times \omega_{1}\right)}^{2} .
\end{aligned}
$$

From (7.4) we then obtain

$$
\begin{aligned}
\left\|h^{\frac{1}{2}} \mathrm{e}^{\varphi / h} u\right\|_{L^{2}(Q)}^{2}+\left\|h^{3 / 2} \mathrm{e}^{\varphi / h} \nabla_{x} u\right\|_{L^{2}(Q)}^{2} \leq C\left(\left\|h^{2} \mathrm{e}^{\varphi / h} P u\right\|_{L^{2}(Q)}^{2}+\left(\varepsilon T^{2}\right)^{3}\left\|h^{\frac{1}{2}} \mathrm{e}^{\varphi / h} u\right\|_{L^{2}(Q)}^{2}\right. \\
\left.+\varepsilon T^{2}\left\|h^{3 / 2} \mathrm{e}^{\varphi / h} \nabla_{x} u\right\|_{L^{2}(Q)}^{2}+\left\|h^{\frac{1}{2}} \mathrm{e}^{\varphi / h} u\right\|_{L^{2}\left((0, T) \times \omega_{1}\right)}^{2}+\left\|h^{3 / 2} \mathrm{e}^{\varphi / h} \nabla_{x} u\right\|_{L^{2}\left((0, T) \times \omega_{1}\right)}^{2}\right) .
\end{aligned}
$$


For $\varepsilon T^{2}$ sufficiently small we have

$$
\begin{gathered}
\left\|h^{\frac{1}{2}} \mathrm{e}^{\varphi / h} u\right\|_{L^{2}(Q)}^{2}+\left\|h^{3 / 2} \mathrm{e}^{\varphi / h} \nabla_{x} u\right\|_{L^{2}(Q)}^{2} \leq C\left(\left\|h^{2} \mathrm{e}^{\varphi / h} P u\right\|_{L^{2}(Q)}^{2}+\left\|h^{\frac{1}{2}} \mathrm{e}^{\varphi / h} u\right\|_{L^{2}\left((0, T) \times \omega_{1}\right)}^{2}\right. \\
\left.+\left\|h^{3 / 2} \mathrm{e}^{\varphi / h} \nabla_{x} u\right\|_{L^{2}\left((0, T) \times \omega_{1}\right)}^{2}\right)
\end{gathered}
$$

We now aim to remove the last term in the r.h.s. of the previous estimation. Let $\chi \in \mathscr{C}_{c}^{\infty}(\omega)$ be such that $\chi=1$ in a neighborhood of $\bar{\omega}_{1}$. If $P u=f$, after multiplication by $\mathrm{e}^{2 \varphi / h} h^{3} \chi \bar{u}$, and integration over $Q$, we obtain

$$
\frac{1}{2} \iint_{Q} \mathrm{e}^{2 \varphi / h} h^{3} \chi \partial_{t}|u|^{2} \mathrm{~d} t \mathrm{~d} x-\operatorname{Re} \iint_{Q} \mathrm{e}^{2 \varphi / h} h^{3} \chi \bar{u} \Delta u \mathrm{~d} t \mathrm{~d} x=\operatorname{Re} \iint_{Q} \mathrm{e}^{2 \varphi / h} h^{3} \chi \bar{u} f \mathrm{~d} t \mathrm{~d} x .
$$

For the first term $I_{1}$ an integration by parts in $t$ yields

$$
\begin{aligned}
\left|I_{1}\right| & =\left.\left|\frac{1}{2} \iint_{Q} \mathrm{e}^{2 \varphi / h} h^{3} \chi \partial_{t}\right| u\right|^{2} \mathrm{~d} t \mathrm{~d} x|=| \frac{1}{2} \iint_{Q}\left(3 \varepsilon \theta^{\prime} h^{2}-2 \varphi \varepsilon \theta^{\prime} h\right) \mathrm{e}^{2 \varphi / h} \chi|u|^{2} \mathrm{~d} t \mathrm{~d} x \mid \\
& \leq C\left\|h^{\frac{1}{2}} \mathrm{e}^{\varphi / h} u\right\|_{L^{2}((0, T) \times \omega)}^{2},
\end{aligned}
$$

since $\varepsilon\left|\theta^{\prime}\right| \leq C \varepsilon T$ is bounded. The third term can be estimated as

$$
\left|I_{3}\right|=\left|\operatorname{Re} \iint_{Q} \mathrm{e}^{2 \varphi / h} h^{3} \chi \bar{u} f \mathrm{~d} t \mathrm{~d} x\right| \leq C\left\|h^{2} \mathrm{e}^{\varphi / h} f\right\|_{L^{2}(Q)}^{2}+C\left\|h^{\frac{1}{2}} \mathrm{e}^{\varphi / h} u\right\|_{L^{2}((0, T) \times \omega)}^{2} .
$$

For the second term, with integration by parts in $x$, we have

$$
\begin{aligned}
I_{2} & =\iint_{Q} \mathrm{e}^{2 \varphi / h} h^{3} \chi\left|\nabla_{x} u\right|^{2} \mathrm{~d} t \mathrm{~d} x+\operatorname{Re} \iint_{Q} h^{3} \nabla_{x}\left(\mathrm{e}^{2 \varphi / h} \chi\right) \bar{u} \nabla_{x} u \mathrm{~d} t \mathrm{~d} x \\
& \geq\left\|h^{\frac{3}{2}} \mathrm{e}^{\varphi / h} \nabla_{x} u\right\|_{L^{2}\left((0, T) \times \omega_{1}\right)}^{2}-\frac{1}{2} \iint_{Q} h^{3} \Delta\left(\mathrm{e}^{2 \varphi / h} \chi\right)|u|^{2} \mathrm{~d} t \mathrm{~d} x
\end{aligned}
$$

and $\left.\left|\iint_{Q} h^{3} \Delta\left(\mathrm{e}^{2 \varphi / h} \chi\right)\right| u\right|^{2} \mathrm{~d} t \mathrm{~d} x \mid \leq C\left\|h^{\frac{1}{2}} \mathrm{e}^{\varphi / h} u\right\|_{L^{2}((0, T) \times \omega)}^{2}$. The previous estimates and (7.5) then yield

$$
\left\|h^{\frac{3}{2}} \mathrm{e}^{\varphi / h} \nabla_{x} u\right\|_{L^{2}\left((0, T) \times \omega_{1}\right)}^{2} \leq C\left\|h^{2} \mathrm{e}^{\varphi / h} P u\right\|_{L^{2}(Q)}^{2}+C\left\|h^{\frac{1}{2}} \mathrm{e}^{\varphi / h} u\right\|_{L^{2}((0, T) \times \omega)}^{2}
$$

The proof is complete.

\subsection{Observability inequality and controllability}

It is now simple to deduce an observability inequality for the adjoint system

$$
\begin{cases}-\partial_{t} q-\Delta q=0 & \text { in } Q \\ q=0 & \text { on } \Sigma \\ q(T)=q_{T} & \text { in } \Omega\end{cases}
$$


We note that the estimate of Theorem 7.8 also applies to the adjoint operator $-\partial_{t}-\Delta$. With the parabolic decay of energy we have $\frac{1}{2} T\|q(0)\|_{L^{2}(\Omega)}^{2} \leq\|q\|_{L^{2}((T / 4,3 T / 4) \times \Omega)}^{2}$. We also have $C \mathrm{e}^{-C^{\prime} /\left(\varepsilon T^{2}\right)}\|q\|_{L^{2}((T / 4,3 T / 4) \times \Omega)}^{2} \leq$ $\left\|h^{\frac{1}{2}} \mathrm{e}^{\varphi / h} q\right\|_{L^{2}((T / 4,3 T / 4) \times \Omega)}^{2}$ since we have $0<C T^{2} \leq t(T-t) \leq C^{\prime} T^{2}$ on the interval $[T / 4,3 T / 4]$ (we note that $\varphi$ was chosen negative here, which explains the restriction to the interval $[T / 4,3 T / 4]$ away from 0 and $T$ for this estimation). Then for $\left(T+T^{2}\right) \varepsilon=\delta_{4}$, the Carleman estimate yields

$$
\|q(0)\|_{L^{2}(\Omega)}^{2} \leq \frac{C}{T} \mathrm{e}^{C /\left(\varepsilon T^{2}\right)}\|q\|_{L^{2}((0, T) \times \omega)}^{2} \leq \mathrm{e}^{C+C^{\prime} / T}\|q\|_{L^{2}((0, T) \times \omega)}^{2} .
$$

From this observability inequality we can also deduce the null controllability of the heat equation and obtain Theorem 6.2 again. We note however that we have a more explicit expression for the observability constant including its dependency in the control time $T$. We naturally see the blow up of this constant as $T$ goes to zero.

Remark 7.9. As mentionned in the introduction, parabolic Carleman estimates allow to treat the controllability of more general parabolic equations. By linearization and with a fix point argument, one may consider the controllability of semi-linear parabolic equations for certain forms of non linearities [4,14,18]. A fine knowledge of the observability constant, obtained by parabolic Carleman estimates, is precisely what allows to treat these non linear cases. In particular, the powers of the semi-classical parameter $h$ in the global Carleman estimate of Theorem 7.8 play a central role in these results. Proposition 7.5 shows the optimality of these powers.

\section{Appendix A. Some Additional Results And PRoOfs of intermediate Results}

\section{A.1. Proof of the Gårding inequality}

The symbol $a(x, \xi, h)$ is of the form $a(x, \xi, h)=a_{m}(x, \xi, h)+h a_{m-1}(x, \xi, h)$, with $a_{m-1} \in S^{m-1}$. For $h$ sufficiently small, say $h<h_{1}$, the full symbol $a(x, \xi, h)$ satisfies

$$
\operatorname{Re} a(x, \xi, h) \geq C^{\prime \prime}\langle\xi\rangle^{m}, \quad x \in K, \xi \in \mathbb{R}^{n}, h \in\left(0, h_{1}\right),
$$

with $C^{\prime}<C^{\prime \prime}<C$. Let $U$ be a neighborhood of $K$ such that the previous inequality holds for $(x, \xi) \in U \times \mathbb{R}^{n}$ with the constant $C^{\prime \prime}$ replaced by $C^{\prime \prime \prime}$ that satisfies $C^{\prime}<C^{\prime \prime \prime}<C^{\prime \prime}<C$. Let $\chi(x) \in \mathscr{C}_{c}^{\infty}(U)$ be such that $0 \leq \chi \leq 1$ and $\chi=1$ in a neighborhood of $K$. We then set $\tilde{a}(x, \xi, h)=\chi(x) a(x, \xi, h)+C^{\prime \prime \prime}(1-\chi)(x)\langle\xi\rangle^{m}$ that satisfies

$$
\tilde{a} \in S^{m} \quad \text { and } \quad \operatorname{Re} \tilde{a}(x, \xi, h) \geq C^{\prime \prime \prime}\langle\xi\rangle^{m}, \quad x \in \mathbb{R}^{n}, \xi \in \mathbb{R}^{n}, h \in\left(0, h_{1}\right) .
$$

We moreover note that $(\mathrm{Op}(\tilde{a}) u, u)=(\mathrm{Op}(a) u, u)$ if $\operatorname{supp}(u) \subset K$. Without any loss of generality we may thus consider that the symbol $a$ satisfies (A.1) in the remaining of the proof.

We then choose $L>0$ such that $C^{\prime}<L<C^{\prime \prime \prime}$ and we set

$$
b(x, \xi, h):=\left(\operatorname{Re} a(x, \xi, h)-L\langle\xi\rangle^{m}\right)^{\frac{1}{2}}, \quad \text { and } B=\operatorname{Op}(b) .
$$

The $\psi \mathrm{DO}$ symbolic calculus gives $B^{*} \circ B=\operatorname{Re} \mathrm{Op}(a)-L \Lambda^{m}+h R$, with $R \in \Psi^{m-1}$, where $\operatorname{Re} \operatorname{Op}(a)$ actually means $\left(\mathrm{Op}(a)+\mathrm{Op}(a)^{*}\right) / 2$. We then have

$$
\begin{aligned}
\operatorname{Re}(\operatorname{Op}(a) u, u)=(\operatorname{Re} \mathrm{Op}(a) u, u) \geq L\left(\Lambda^{m} u, u\right)-h(R u, u) & \geq L\left\|\Lambda^{m / 2} u\right\|_{0}^{2}-h L^{\prime}\|u\|_{(m-1) / 2}^{2} \\
& \geq\left(L-h L^{\prime}\right)\|u\|_{m / 2}^{2} .
\end{aligned}
$$

We conclude the proof by taking $h$ sufficiently small. 


\section{A.2. Example of functions fulfilling the sub-ellipticity condition: proof of Lemma 3.3}

We shall actually prove the following stronger lemma here.

Lemma A.1. Let $V$ be a bounded open set in $\mathbb{R}^{n}$ and $\psi \in \mathscr{C}^{\infty}\left(\mathbb{R}^{n}, \mathbb{R}\right)$ be such that $\left|\psi^{\prime}\right|>0$ in $\bar{V}$. Then for $\lambda>0$ sufficiently large, $\varphi=\mathrm{e}^{\lambda \psi}$ satisfies $\left|\varphi^{\prime}\right| \geq C>0$ in $\bar{V}$ and

$$
\forall(x, \xi) \in \bar{V} \times \mathbb{R}^{n}, \quad q_{2}(x, \xi)=0 \quad \Rightarrow \quad\left\{q_{2}, q_{1}\right\}(x, \xi) \geq C>0 .
$$

Proof The computation of the Poisson bracket $\left\{q_{2}, q_{1}\right\}=\sum_{j} \partial_{\xi_{j}} q_{2} \partial_{x_{j}} q_{1}-\partial_{x_{j}} q_{2} \partial_{\xi_{j}} q_{1}$ gives

$$
\left\{q_{2}, q_{1}\right\}=4 \sum_{1 \leq j, k \leq n} \varphi_{j, k}^{\prime \prime}\left(\xi_{j} \xi_{k}+\varphi_{j}^{\prime} \varphi_{k}^{\prime}\right)=4\left(\varphi^{\prime \prime}(\xi, \xi)+\varphi^{\prime \prime}\left(\varphi^{\prime}, \varphi^{\prime}\right)\right)
$$

Here we have $\varphi=\mathrm{e}^{\lambda \psi}$, and thus $\varphi^{\prime}=\lambda \varphi \psi^{\prime}$ and $\varphi_{j k}^{\prime \prime}=\lambda \varphi \psi_{j k}^{\prime \prime}+\lambda^{2} \varphi \psi_{j}^{\prime} \psi_{k}^{\prime}, j, k=1, \ldots, n$, which yields

$$
\left\{q_{2}, q_{1}\right\}=4 \lambda^{3} \varphi^{3}\left(\lambda\left|\psi^{\prime}\right|^{4}+\psi^{\prime \prime}\left(\psi^{\prime}, \psi^{\prime}\right)+\psi^{\prime \prime}\left((\lambda \varphi)^{-1} \xi,(\lambda \varphi)^{-1} \xi\right)+\lambda^{-1} \varphi^{-2}\left\langle\psi^{\prime}, \xi\right\rangle^{2}\right) .
$$

When $q_{2}=0$ we have $|\xi|=\lambda \varphi\left|\psi^{\prime}\right|$. We then note that

$$
\left|\psi^{\prime \prime}\left((\lambda \varphi)^{-1} \xi,(\lambda \varphi)^{-1} \xi\right)\right| \leq C\left|\psi^{\prime}\right|^{2}, \quad\left|\psi^{\prime \prime}\left(\psi^{\prime}, \psi^{\prime}\right)\right| \leq C\left|\psi^{\prime}\right|^{2} .
$$

We deduce

$$
\left\{q_{2}, q_{1}\right\} \geq 4 \lambda^{3} \varphi^{3}\left(\lambda\left|\psi^{\prime}\right|^{4}-C\left|\psi^{\prime}\right|^{2}\right) .
$$

We then see that for $\lambda$ sufficiently large we have $\left\{q_{2}, q_{1}\right\} \geq C_{\lambda}>0$, since $\left|\psi^{\prime}\right| \geq C>0$.

Remark A.2. In Lemma 3.3 we chose to use an exponential function. The reader will note that a similar result can be obtained by taking $\varphi=G(\lambda \psi)$, with $\lambda$ sufficiently large, for a function $G: \mathbb{R} \rightarrow \mathbb{R}$ that satisfies $G^{\prime}>0, G^{\prime \prime}>0$ and $G^{\prime \prime} / G^{\prime} \geq C>0$. This procedure is often referred to as the "convexification" of the weight function.

\section{A.3. Proof of Lemma 3.4}

For $|\xi|$ large, the property holds since $q_{2}=|\xi|^{2}-\left|\varphi^{\prime}\right|^{2}$ and since the symbol $\left\{q_{2}, q_{1}\right\}$ is only of order 2 .

It remains to prove the result for $|\xi| \leq R$, with $R>0$, i.e. for $(x, \xi)$ in a compact set (here $x \in \bar{V}$ ). In a more general framework, consider two continuous functions, $f$ and $g$, defined in a compact set $\mathcal{K}$, and assume that $f \geq 0$ and $f(y)=0 \Rightarrow g(y) \geq L>0$. We set $h_{\mu}=\mu f+g$.

For all $y \in \mathcal{K}$, either $f(y)=0$ and thus $h_{\mu}(y)>L$, or $f(y)>0$ and thus there exists $\mu_{y}>0$ such that $h_{\mu_{y}}(y)>0$. This inequality holds locally in an open neighborhood $V_{y}$ of y. From the covering of $\mathcal{K}$ by the open sets $V_{y}$, we select a finite covering $V_{y_{1}}, \ldots, V_{y_{n}}$ and set $\mu=\max _{1 \leq j \leq n} \mu_{j}$. We then obtain $h_{\mu} \geq C>0$. We simply apply this result to $\rho /\langle\xi\rangle^{4}$.

\section{A.4. Proof of Lemma 3.10}

We saw in Section A.2 that

$$
\left\{q_{2}, q_{1}\right\}=4 \lambda^{3} \varphi^{3}\left(\lambda\left|\psi^{\prime}\right|^{4}+\psi^{\prime \prime}\left(\psi^{\prime}, \psi^{\prime}\right)+\psi^{\prime \prime}\left((\lambda \varphi)^{-1} \xi,(\lambda \varphi)^{-1} \xi\right)+\lambda^{-1} \varphi^{-2}\left\langle\psi^{\prime}, \xi\right\rangle^{2}\right) .
$$


We observe that $q_{2} \Delta \varphi=\left(|\xi|^{2}-\lambda^{2}\left|\psi^{\prime}\right|^{2} \varphi^{2}\right)\left(\lambda^{2}\left|\psi^{\prime}\right|^{2} \varphi+\lambda(\Delta \psi) \varphi\right)$, which yields

$$
\begin{aligned}
\rho=\lambda^{3} \varphi^{3}( & 4 \psi^{\prime \prime}\left((\lambda \varphi)^{-1} \xi,(\lambda \varphi)^{-1} \xi\right)+2 \mu\left(\lambda\left|\psi^{\prime}\right|^{2}+\Delta \psi\right)\left|\frac{\xi}{\lambda \varphi}\right|^{2}+\lambda^{-1} \varphi^{-2}\left\langle\psi^{\prime}, \xi\right\rangle^{2} \\
& \left.+(4-2 \mu) \lambda\left|\psi^{\prime}\right|^{4}+4 \psi^{\prime \prime}\left(\psi^{\prime}, \psi^{\prime}\right)-2 \mu\left|\psi^{\prime}\right|^{2} \Delta \psi\right)
\end{aligned}
$$

which, as $0<\mu<2$, we can make larger than $C_{\lambda}\langle\xi\rangle^{2}$, with $C_{\lambda}>0$ by taking $\lambda$ sufficiently large.

\section{A.5. Estimations in the elliptic region and close to the characteristic set: proof of Proposition 3.8}

Let $w_{1}=\mathrm{Op}\left(\chi_{1}\right) v$. We observe that $\operatorname{supp}\left(w_{1}\right) \subset V$ from the assumption made on the support of the symbol $\chi_{1}$. Then $P_{\varphi} w_{1}=g_{1}=\operatorname{Op}\left(\chi_{1}\right) g+\left[P_{\varphi}, \mathrm{Op}\left(\chi_{1}\right)\right] v$, where $g=P_{\varphi} v$. The commutator is in $h \Psi^{1}$ and thus

$$
\left\|g_{1}\right\|_{0} \leq C\left(\|g\|_{0}+h\|v\|_{1}\right) .
$$

Let $\chi \in S^{0}$ be such that $\chi=1$ in a neighborhood of $\operatorname{supp}\left(\chi_{1}\right)$ and $\chi=0$ in a neighborhood of $\mathscr{Z}$. Because of the ellipticity of $P_{\varphi}$ in $\operatorname{supp}(\chi)$, there exists a $\psi \mathrm{DO}$ parametrix (see [27,42]) $E_{M}=\mathrm{Op}(e), e \in S^{-2}$, with $e=\sum_{j=0}^{M} h^{j} e_{j}$, for $M \in \mathbb{N}$ large, where $e_{j} \in S^{2-j}, e_{0}=\chi / p_{\varphi}$, and

$$
E_{M} \circ P_{\varphi}=\mathrm{Op}(\chi)+h^{M+1} R_{M}, \quad R_{M} \in \Psi^{-1-M} .
$$

We then obtain $w_{1}=E_{M} g_{1}+\tilde{g}_{1}$, with $\tilde{g}_{1}=(\operatorname{Id}-\mathrm{Op}(\chi)) w_{1}-h^{M+1} R_{M} w_{1}$. As $\operatorname{supp}(1-\chi) \cap \operatorname{supp}\left(\chi_{1}\right)=\emptyset$, and $w_{1}=\mathrm{Op}\left(\chi_{1}\right) v$, the $\psi \mathrm{DO}$ calculus of Theorem 2.8 yields

$$
\left\|\tilde{g}_{1}\right\|_{2} \leq C h\|v\|_{1} .
$$

With (A.3) and (A.4) we obtain the first result of the proposition,

$$
\left\|w_{1}\right\|_{2} \leq C\left(\left\|g_{1}\right\|_{0}+h\|v\|_{1}\right) \leq C^{\prime}\left(\|g\|_{0}+h\|v\|_{1}\right) .
$$

For the second part we introduce $w_{2}=\mathrm{Op}\left(\chi_{2}\right) v$. We then have $P_{\varphi} w_{2}=g_{2}=\mathrm{Op}\left(\chi_{2}\right) g+\left[P_{\varphi}, \mathrm{Op}\left(\chi_{2}\right)\right] v$ and $\left\|g_{2}\right\|_{0} \leq C\left(\|g\|_{0}+h\|v\|_{1}\right)$. The proof of the Carleman estimate then yields (see (3.3)) $h\left\|w_{2}\right\|_{2}^{2} \leq C\left(\|g\|_{0}^{2}+h^{2}\|v\|_{1}^{2}\right)$.

Let $\tilde{\chi}_{2} \in S^{0}$ with compact support be such that $\tilde{\chi}_{2}=1$ in a neighborhood of $\operatorname{supp}\left(\chi_{2}\right)$. The $\psi \mathrm{DO}$ calculus of Theorem 2.8 yields

$$
w_{2}=\mathrm{Op}\left(1-\tilde{\chi}_{2}\right) w_{2}+\mathrm{Op}\left(\tilde{\chi}_{2}\right) w_{2}=\underbrace{\mathrm{Op}\left(1-\tilde{\chi}_{2}\right) \operatorname{Op}\left(\chi_{2}\right)}_{\in h^{N} \Psi^{-N}} v+\mathrm{Op}\left(\tilde{\chi}_{2}\right) w_{2}, \quad N \in \mathbb{N},
$$

and we thus obtain, for all $N \in \mathbb{N}$ and $r, r^{\prime} \in \mathbb{R}$,

$$
\left\|w_{2}\right\|_{s}=\left\|\Lambda^{s} w_{2}\right\|_{0} \leq C_{N, r, s} h^{N}\|v\|_{r}+\|\underbrace{\Lambda^{s} \mathrm{Op}\left(\tilde{\chi}_{2}\right)}_{\in \Psi^{r^{\prime}}} w_{2}\|_{0} \leq C_{N, r, s} h^{N}\|v\|_{r}+C_{r^{\prime}, s}^{\prime}\left\|w_{2}\right\|_{r^{\prime}}
$$

It follows that $\left\|w_{2}\right\|_{s} \leq C_{s} h\|v\|_{1}+C_{s}^{\prime}\left\|w_{2}\right\|_{2}$, for all $s \in \mathbb{R}$, which yields the result. 


\section{A.6. On the sub-ellipticity condition and the optimality of the powers of the semi-classical parameter: proof of Proposition 3.9}

The proof that we give is adapted from that of Theorem 8.1.1 in [25]. We refer the reader to this proof for the treatment of more general operators. Here, the symbol of the Laplace operator does not depend on $x$, which simplifies the proof.

Let $x_{0} \in V$ and let $\xi_{0} \in \mathbb{R}^{n}$ be such that $p_{\varphi}\left(x_{0}, \xi_{0}\right)=0$ (such a $\xi_{0}$ always exists). There is no restriction to consider the case $x_{0}=0$ and $\varphi\left(x_{0}\right)=0$. We set $\zeta_{0}=\xi_{0}+i \varphi^{\prime}\left(x_{0}\right)$. Then $\sum_{j} \zeta_{0, j}^{2}=0$. We set $w(x)=\left\langle x, \zeta_{0}\right\rangle$ and have

$$
\varphi(x)-\operatorname{Im}(w(x))=A(x)+o\left(|x|^{2}\right), \quad \text { with } \quad A(x)=\frac{1}{2} \sum_{j, k} \varphi_{j k}^{\prime \prime}\left(x_{0}\right) x_{j} x_{k}
$$

Let $\phi \in \mathscr{C}_{c}^{\infty}\left(\mathbb{R}^{n}\right)$ be such that $\phi(0) \neq 0$. We introduce $u_{h}=\mathrm{e}^{\mathrm{i} w(x) / h} \phi\left(x / h^{\frac{1}{2}}\right)$ and we have

$$
h^{2} P u_{h}=\mathrm{e}^{\mathrm{i} w / h}\left(-h(\Delta \phi)\left(x / h^{\frac{1}{2}}\right)-\mathrm{i} h \Delta w(x) \phi\left(x / h^{\frac{1}{2}}\right)+\left(\sum_{j}\left(\partial_{x_{j}} w(x)\right)^{2}\right) \phi\left(x / h^{\frac{1}{2}}\right)-2 \mathrm{i} h^{\frac{1}{2}}\left\langle\nabla_{x} w(x), \phi^{\prime}\left(x / h^{\frac{1}{2}}\right)\right\rangle\right) .
$$

We note that $\sum_{j}\left(\partial_{x_{j}} w(x)\right)^{2}=0$ and observe that we then have

$$
\left\|h^{2} \mathrm{e}^{\varphi / h} P u_{h}\right\|_{0}^{2}=h^{n / 2} \int \mathrm{e}^{\frac{2}{h}\left(A\left(h^{\frac{1}{2}} x\right)+o\left(\left|h^{\frac{1}{2}} x\right|^{2}\right)\right)}\left|-h \Delta \phi(x)-\mathrm{i} h(\Delta w)\left(h^{\frac{1}{2}} x\right) \phi(x)-2 \mathrm{i} h^{\frac{1}{2}}\left\langle(\nabla w)\left(h^{\frac{1}{2}} x\right), \phi^{\prime}(x)\right\rangle\right|^{2} \mathrm{~d} x .
$$

after the change of variables $x \rightarrow x / h^{\frac{1}{2}}$. In the limit $h \rightarrow 0$, by dominated convergence, we have

$$
\left\|h^{2} \mathrm{e}^{\varphi / h} P u_{h}\right\|_{0}^{2} \sim 4 h^{(n / 2+1)} \int \mathrm{e}^{2 A(x)}\left|\left\langle\zeta_{0}, \phi^{\prime}(x)\right\rangle\right|^{2} \mathrm{~d} x .
$$

Similarly we have $h^{2 \alpha}\left\|\mathrm{e}^{\varphi / h} u_{h}\right\|_{0}^{2} \sim h^{(n / 2+2 \alpha)} \int \mathrm{e}^{2 A(x)}|\phi(x)|^{2} \mathrm{~d} x$. With Inequality (3.7) we conclude that we necessarily have $\alpha=\frac{1}{2}$ and $\left\langle\zeta_{0}, \phi^{\prime}(x)\right\rangle \not \equiv 0$. In particular $\varphi^{\prime}\left(x_{0}\right) \neq 0$ since otherwise we would have $\zeta_{0}=0$.

If we denote by $K$ the constant $C$ in (3.7), with $\alpha=\frac{1}{2}$, the limit $h \rightarrow 0$ yields

$$
\int \mathrm{e}^{2 A(x)}|\phi(x)|^{2} \mathrm{~d} x \leq K \int \mathrm{e}^{2 A(x)}\left|\left\langle\zeta_{0}, \phi(x)^{\prime}\right\rangle\right|^{2} \mathrm{~d} x
$$

for all $\phi \in \mathscr{C}_{c}^{\infty}\left(\mathbb{R}^{n}\right)$. Lemma 8.1.3 in [25] then gives $\sum_{j, k} \varphi_{j k}^{\prime \prime} \zeta_{0, j} \overline{\zeta_{0, k}} \geq \frac{1}{2 K}$. We set $\zeta=\xi+i \varphi^{\prime}(x)$. The computation carried out in Section A.2 gives $\left\{q_{2}, q_{1}\right\}=4 \varphi^{\prime \prime}(\zeta, \zeta)=4 \sum_{j, k} \varphi_{j, k}^{\prime \prime} \zeta_{j} \overline{\zeta_{k}}$. For all $x \in V$ we thus have

$$
\xi \in \mathbb{R}^{n} \text { and } p_{\varphi}(x, \xi)=0 \Rightarrow\left\{q_{2}, q_{1}\right\}(x, \xi) \geq \frac{2}{K}
$$

Let now $(y, \eta) \in \partial V \times \mathbb{R}^{n}$ be such that $p_{\varphi}(y, \eta)=0$. We first consider a sequence $\left(x^{(k)}\right)_{k \in \mathbb{N}^{*}} \subset V$ that converges to $y$ and $\left(\xi^{(k)}\right)_{k \in \mathbb{N}^{*}} \subset \mathbb{R}^{n}$ such that $p_{\varphi}\left(x^{(k)}, \xi^{(k)}\right)=0$. We set $\zeta^{(k)}=\xi^{(k)}+i \varphi^{\prime}\left(x^{(k)}\right)$. We have in particular $\left|\xi^{(k)}\right|=\left|\varphi^{\prime}\left(x^{(k)}\right)\right|$ and the sequence $\left(\xi^{(k)}\right)_{k}$ is hence bounded. It converges, up to a sub-sequence, to a certain $\xi \in \mathbb{R}^{n}$ and thus $p_{\varphi}(y, \xi)=0$. In particular $|\xi|=\left|\varphi^{\prime}(y)\right|$. We saw above that $\left\{q_{2}, q_{1}\right\}\left(x^{(k)}, \xi^{(k)}\right)=$ $4 \sum_{i, j} \varphi_{i, j}^{\prime \prime}\left(x^{(k)}\right) \zeta_{i}^{(k)} \overline{\zeta_{j}^{(k)}} \geq \frac{2}{K}$. If $\zeta=\xi+i \varphi^{\prime}(y)$ we have $\left\{q_{2}, q_{1}\right\}(y, \xi)=4 \sum_{i, j} \varphi_{i, j}^{\prime \prime}(y) \zeta_{i} \overline{\zeta_{j}} \geq \frac{2}{K}$. This excludes $\zeta=0$. As $|\xi|=\left|\varphi^{\prime}(y)\right|$ we obtain that $\varphi^{\prime}(y) \neq 0$.

The characteristic set over $\bar{V}$ is given by $\mathscr{Z}=\tilde{\mathscr{Z}} \cap\left(\bar{V} \times \mathbb{R}^{n}\right)$ with

$$
\tilde{\mathscr{Z}}=\left\{(x, \xi) \in \mathbb{R}^{n} \times \mathbb{R}^{n} ; f_{1}(x, \xi)=|\xi|^{2}-\left|\varphi^{\prime}(x)\right|^{2}=0 \text {, and } f_{2}(x, \xi)=\left\langle\xi, \varphi^{\prime}(x)\right\rangle=0\right\} .
$$


As $\varphi^{\prime}(y) \neq 0$ and $\eta \neq 0$ is orthogonal to $\varphi^{\prime}(y)$, we then see that the partial differentials $d_{\xi} f_{1}$ and $d_{\xi} f_{2}$ form a rank 2 system at $(y, \eta)$. Up to rearranging the variables, with the implicit function theorem, this implies that in a neighborhood $U_{1}$ of $(y, \eta)$ and in a neighborhood $U_{2}$ of $\left(y, \eta_{1}, \ldots, \eta_{n-2}\right)$ we have

$$
(x, \xi) \in \tilde{\mathscr{Z}} \cap U_{1} \Leftrightarrow\left(x, \xi_{1}, \ldots, \xi_{n-2}\right) \in U_{2} \text { and }\left(\xi_{n-1}, \xi_{n}\right)=g\left(x, \xi_{1}, \ldots, \xi_{n-2}\right),
$$

with a smooth function $g$. Consider then a sequence $\left(x^{(k)}\right)_{k \in \mathbb{N}^{*}} \subset V$ that converges to $y$. For $k$ sufficiently large, $k \geq N_{0}$, we have $\left(x^{(k)}, \eta_{1}, \ldots, \eta_{n-2}\right) \in U_{2}$ and we set $\xi^{(k)}=\left(\eta_{1}, \ldots, \eta_{n-2}, g\left(x^{(k)}, \eta_{1}, \ldots, \eta_{n-2}\right)\right)$. Then $\left(x^{(k)}, \xi^{(k)}\right)$ is in $\mathscr{Z}$ and converge to $(y, \eta)$. We have $\left\{q_{2}, q_{1}\right\}\left(x^{(k)}, \xi^{(k)}\right) \geq 2 / K$ for all $k \geq N_{0}$ by the first part of the proof. We thus obtain $\left\{q_{2}, q_{1}\right\}(y, \eta) \geq 2 / K$ by passing to the limit.

Remark A.3. In the previous proof we have chosen a test function $u_{h}$ that is localized around $x_{0}$ in space, through the term $\phi\left(x / h^{\frac{1}{2}}\right)$, and around $\zeta_{0}$ in frequencies, through the term $\mathrm{e}^{\mathrm{i}\left\langle x, \zeta_{0}\right\rangle / h}$, for the semi-classical Fourier transformation. This microlocalization shows clearly that the non-zero power of the parameter $h$ in the 1.h.s. of the Carleman estimate originates from the behavior of the symbol at the characteristic set $\mathscr{Z}$ as we already pointed out with Proposition 3.8. We note that the scaling $x / h^{\frac{1}{2}}$ in $\phi\left(x / h^{\frac{1}{2}}\right)$ allows to control the variations of $A(x) / h$ in the support of $u_{h}$.

\section{A.7. Estimation of the heat kernel}

Let $p_{t}(x, y)$ be the heat kernel in $\Omega$, a bounded open set in $\mathbb{R}^{n}$, with homogeneous Dirichlet boundary conditions.

Lemma A.4. For all $x, y \in \Omega$ we have $p_{t}(x, y) \leq(4 \pi t)^{-n / 2} \mathrm{e}^{-\frac{|x-y|^{2}}{4 t}}$ if $t>0$.

Proof The heat kernel in $\mathbb{R}^{n}$ is given by $p_{0, t}(x, y)=(4 \pi t)^{-n / 2} \mathrm{e}^{-\frac{|x-y|^{2}}{4 t}}$ if $t>0$. Consider $y_{0} \in \mathscr{C}_{c}^{\infty}(\Omega)$ such that $y_{0} \geq 0$. We also denote by $y_{0}$ its zero extension to $\mathbb{R}^{n}$. We consider the following parabolic problems

$$
\left\{\begin{array} { l l } 
{ \partial _ { t } y - \Delta y = 0 } & { \text { in } ( 0 , T ) \times \Omega , } \\
{ y = 0 } & { \text { on } ( 0 , T ) \times \partial \Omega , } \\
{ y | _ { t = 0 } = y _ { 0 } } & { \text { in } \Omega , }
\end{array} \quad \left\{\begin{array}{ll}
\partial_{t} z-\Delta z=0 & \text { in }(0, T) \times \mathbb{R}^{n}, \\
\left.z\right|_{t=0}=y_{0} & \text { in } \mathbb{R}^{n} .
\end{array}\right.\right.
$$

For $t>0$, the solutions $z$ and $y$ are smooth functions given by $y(t, x)=\left\langle p_{t}(x,),. y_{0}().\right\rangle$ and $z(t, x)=$ $\left\langle p_{0, t}(x,),. y_{0}().\right\rangle$. In particular $z(t, x) \geq 0$ if $x \in \partial \Omega$. Thus $z-y \geq 0$ in $(0, T) \times \partial \Omega$. The difference of the two solutions thus satisfies a parabolic problem of the following form

$$
\begin{cases}\partial_{t}(z-y)-\Delta(z-y)=0 & \text { in }(0, T) \times \Omega \\ z-y \geq 0 & \text { on }(0, T) \times \partial \Omega \\ \left.(z-y)\right|_{t=0}=0 & \text { in } \Omega\end{cases}
$$

The maximum principle gives $z-y \geq 0$ in $(0, T) \times \Omega$ (see e.g. [10], Thm. X.6). If $y_{0} \in \mathscr{C}_{c}^{\infty}(\Omega)$ with $y_{0} \geq 0$, it follows that $\left\langle p_{0, t}(x,),. y_{0}().\right\rangle \geq\left\langle p_{t}(x,),. y_{0}().\right\rangle$ for $t>0$. This yields the result.

Lemma A.5. Let $p_{0, t}(x, y)=(4 \pi t)^{-n / 2} \mathrm{e}^{-\frac{|x-y|^{2}}{4 t}}$ be the heat kernel in $\mathbb{R}^{n}$ and let $y \in \Omega$ and $V_{y}$ be a neighborhood of $y$ such that $\bar{V}_{y} \subset \Omega$. There exists $C$ and $C^{\prime}>0$ such that

$$
\left|p_{t}(x, y)-p_{0, t}(x, y)\right| \leq C t \mathrm{e}^{-C^{\prime} / t}, \quad x \in \bar{V}_{y}, t>0
$$


Proof Let $\chi \in \mathscr{C}_{c}^{\infty}(\Omega)$ be such that $\chi=1$ near $\bar{V}_{y}$. We introduce $v(t, x)=p_{t}(x, y)-\chi(x) p_{0, t}(x, y)$ and observe that $\left.v\right|_{t=0}=0,\left.v\right|_{(0,+\infty) \times \partial \Omega}=0$ and that $v$ satisfies the parabolic equation $\left(\partial_{t}-\Delta\right) v=w$ with

$$
w(t, x)=p_{0, t}(x, y)\left(\Delta \chi(x)-\frac{1}{t}(\nabla \chi(x), x-y)\right) .
$$

We observe that $|x-y| \geq d>0$ in $\operatorname{supp}\left(\chi^{\prime}\right)$ and thus have $\tilde{w}(t, x)=\mathrm{e}^{\frac{d^{2}}{4 t}} w(t, x) \in \mathscr{C}^{\infty}([0,+\infty[\times \Omega)$ with furthermore $\|\tilde{w}\|_{L^{\infty}([0,+\infty[\times \Omega)}<\infty$. The Duhamel formula gives $v(t, x)=\int_{0}^{t} S(t-s) w(s) \mathrm{d} s$, where $S(t)$ is the heat semi-group and we find $v(t)=\int_{0}^{t} \mathrm{e}^{-\frac{d^{2}}{4 s}} S(t-s) \tilde{w}(s) \mathrm{d} s$, which yields

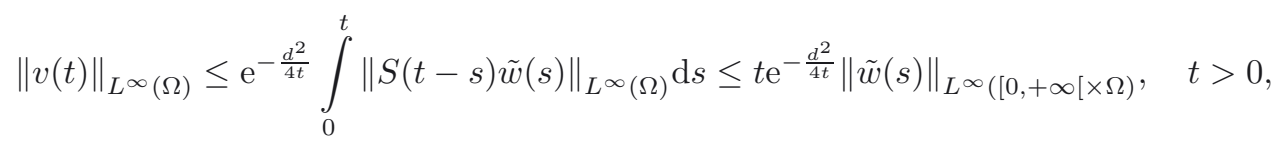

by the maximum principle [10], Theorem X.3. The result follows in $\bar{V}_{y}$ where $\chi=1$.

The reader will note that we can obtain the following short-time asymptotic expansion of the heat kernel on a Riemannian manifold, with or without boundary, on the diagonal, for all $N \in \mathbb{N}$ (see for instance estimate (13.59) along with (13.39) and (13.40) in [56], Chap. 7.13)

$$
P_{t}(y, y)=t^{-n / 2}\left(C_{0}(y)+t C_{1}(y)+\cdots+C_{N}(y) t^{N}+\mathcal{O}\left(t^{N+1}\right)\right), \quad t \rightarrow 0 .
$$

In the case we consider here, the metric is flat, and such an expansion is greatly simplified as shown in the previous lemma.

\section{A.8. Proof of Lemma 7.2}

The proof of Lemma 7.2 is close to that of Lemma 3.4. It suffices to prove the result for $(x, \xi)$ in a compact $\mathcal{K} \subset \mathbb{R}^{n} \times \mathbb{R}^{n}$. We first take $\varepsilon=0$. We have $\left.q_{2}\right|_{\varepsilon=0}=|\xi|^{2}-\left|\varphi^{\prime}\right|^{2}$. For $\mu$ sufficiently large we have

$$
\left.\mu q_{2}^{2}\right|_{\varepsilon=0}+\left\{\left.q_{2}\right|_{\varepsilon=0}, b\right\} \geq C\langle\xi\rangle^{4}, \quad(x, \xi) \in \mathcal{K},
$$

as seen in the proof of Lemma A.1 (p. 735). Finally, since $(x, \xi)$ is in a compact set, this inequality still holds, with a different constant $C$, for $\varepsilon\left|\theta^{\prime}\right|>0$ small. Since $\left|\theta^{\prime}(t)\right| \leq T$, this concludes the proof.

\section{A.9. Proof of Proposition 7.5}

The proof is close to that of Proposition 3.9. Let $x_{0} \in V$ and $\xi_{0} \in \mathbb{R}^{n}$ be such that $\left.q_{2}\right|_{\varepsilon=0}=0$ (such a $\xi_{0}$ always exists). We set $\zeta_{0}=\xi_{0}+i \varphi^{\prime}\left(x_{0}\right)$. There is no restriction to consider the case $x_{0}=0$. We then choose $\tau_{0} \in \mathbb{R}$ such that

$$
\tau_{0}+2\left\langle\varphi^{\prime}\left(x_{0}\right), \xi_{0}\right\rangle=0
$$

We then have

$$
i \tau_{0}+\sum_{j} \zeta_{0, j}^{2}=0
$$

Next, we define

$$
w(t, x)=\underbrace{i \varphi(0)+\left\langle x, \zeta_{0}\right\rangle}_{=\tilde{w}(x)}+\tau_{0}\left(t-t_{0}\right) / h, \quad \text { with } t_{0}=T / 2,
$$


and we have

$$
\varphi(x)-\operatorname{Im}(w(t, x))=A(x)+o\left(|x|^{2}\right), \quad \text { with } A(x)=\sum_{j, k} \varphi_{j k}^{\prime \prime}\left(x_{0}\right) x_{j} x_{k}
$$

Let $\gamma \in(0, T / 2)$ and let $\phi \in \mathscr{C}_{c}^{\infty}\left((\gamma, T-\gamma) \times \mathbb{R}^{n}\right)$ be such that $\phi\left(t_{0}, 0\right) \neq 0$. We then set

$$
u_{\varepsilon}=\mathrm{e}^{\mathrm{i} w(t, x) / h} \phi\left(\frac{t-t_{0}}{\varepsilon}, \frac{x}{\sqrt{\varepsilon}}\right)
$$

We recall that $\theta=t(T-t)$ and $h=\varepsilon \theta$. We have

$$
\partial_{t} u_{\varepsilon}=\mathrm{e}^{\mathrm{i} w / h}\left(i \partial_{t}\left(\frac{w(t, x)}{h}\right) \phi\left(\frac{t-t_{0}}{\varepsilon}, \frac{x}{\sqrt{\varepsilon}}\right)+\varepsilon^{-1}\left(\partial_{t} \phi\right)\left(\frac{t-t_{0}}{\varepsilon}, \frac{x}{\sqrt{\varepsilon}}\right)\right),
$$

with

$$
\partial_{t}\left(\frac{w(t, x)}{h}\right)=\tau_{0} \partial_{t}\left(\frac{t-t_{0}}{h^{2}}\right)+\tilde{w}(x) \partial_{t} h^{-1}=\frac{\tau_{0}}{h^{2}}-2 \tau_{0}\left(t-t_{0}\right) \frac{\theta^{\prime}}{h^{2} \theta}-\tilde{w}(x) \frac{\theta^{\prime}}{h \theta} .
$$

We also have (similarly to the elliptic case in see (A.5)),

$$
\begin{aligned}
-h^{2} \Delta_{x} u_{\varepsilon}= & \mathrm{e}^{\mathrm{i} w / h}\left(\left(\sum_{j}\left(\partial_{x_{j}} \tilde{w}(x)\right)^{2}-\mathrm{i} h \Delta_{x} \tilde{w}(x)\right) \phi\left(\frac{t-t_{0}}{\varepsilon}, \frac{x}{\sqrt{\varepsilon}}\right)-2 \mathrm{i} \varepsilon^{\frac{1}{2}} \theta\left\langle\nabla_{x} \tilde{w}(x), \phi_{x}^{\prime}\left(\frac{t-t_{0}}{\varepsilon}, \frac{x}{\sqrt{\varepsilon}}\right)\right\rangle\right. \\
& \left.-\varepsilon \theta^{2}\left(\Delta_{x} \phi\right)\left(\frac{t-t_{0}}{\varepsilon}, \frac{x}{\sqrt{\varepsilon}}\right)\right) .
\end{aligned}
$$

With (A.7), and as $\theta^{\prime}=2\left(t_{0}-t\right)$ it follows that

$$
\begin{aligned}
h^{2} P u_{\varepsilon}= & \mathrm{e}^{\mathrm{i} w / h}\left(\frac{2 \mathrm{i}}{\theta}\left(2 \tau_{0}\left(t-t_{0}\right)^{2}+h\left(t-t_{0}\right) \tilde{w}(x)\right) \phi\left(\frac{t-t_{0}}{\varepsilon}, \frac{x}{\sqrt{\varepsilon}}\right)+h \theta \partial_{t} \phi\left(\frac{t-t_{0}}{\varepsilon}, \frac{x}{\sqrt{\varepsilon}}\right)\right. \\
& \left.-\mathrm{i} h \Delta_{x} \tilde{w}(x) \phi\left(\frac{t-t_{0}}{\varepsilon}, \frac{x}{\sqrt{\varepsilon}}\right)-2 \mathrm{i} \varepsilon^{\frac{1}{2}} \theta\left\langle\nabla_{x} \tilde{w}(x), \phi_{x}^{\prime}\left(\frac{t-t_{0}}{\varepsilon}, \frac{x}{\sqrt{\varepsilon}}\right)\right\rangle-\varepsilon \theta^{2}\left(\Delta_{x} \phi\right)\left(\frac{t-t_{0}}{\varepsilon}, \frac{x}{\sqrt{\varepsilon}}\right)\right) .
\end{aligned}
$$

After the change of variables $(t, x) \rightarrow\left(\left(t-t_{0}\right) / \varepsilon, x / \varepsilon^{\frac{1}{2}}\right)$, we obtain, for $\varepsilon \rightarrow 0$,

$$
\left\|h^{2} \mathrm{e}^{\varphi / h} P u_{\varepsilon}\right\|_{0}^{2} \sim 4 \varepsilon^{n / 2+2} \iint_{\mathbb{R}^{n+1}} \mathrm{e}^{2 A(x) / \theta\left(t_{0}\right)} \theta\left(t_{0}\right)^{2}\left|\left\langle\zeta_{0}, \phi_{x}^{\prime}(t, x)\right\rangle\right|^{2} \mathrm{~d} t \mathrm{~d} x .
$$

As we have $\left\|h^{\alpha} \mathrm{e}^{\varphi / h} u_{\varepsilon}\right\|_{0}^{2} \sim \varepsilon^{(n / 2+1+2 \alpha)} \iint_{\mathbb{R}^{n+1}} \mathrm{e}^{2 A(x) / \theta\left(t_{0}\right)} \theta\left(t_{0}\right)^{2 \alpha}|\phi(t, x)|^{2} \mathrm{~d} t \mathrm{~d} x$, Inequality (7.3) yields $\alpha=\frac{1}{2}$ and $\zeta_{0} \neq 0$. It follows that $\varphi^{\prime}\left(x_{0}\right) \neq 0$. We then have obtained

$$
\iint_{\mathbb{R}^{n+1}} \mathrm{e}^{2 A(x) / \theta\left(t_{0}\right)}|\phi(t, x)|^{2} \mathrm{~d} t \mathrm{~d} x \leq C \iint_{\mathbb{R}^{n+1}} \mathrm{e}^{2 A(x) / \theta\left(t_{0}\right)}\left|\left\langle\zeta_{0}, \phi_{x}^{\prime}(t, x)\right\rangle\right|^{2} \mathrm{~d} t \mathrm{~d} x,
$$


for all $\phi \in \mathscr{C}_{c}^{\infty}\left((\gamma, T-\gamma) \times \mathbb{R}^{n}\right)$. For $\phi_{2} \in \mathscr{C}_{c}^{\infty}\left(\mathbb{R}^{n}\right)$ we thus find ${ }^{4}$

$$
\int_{\mathbb{R}^{n}} \mathrm{e}^{2 A(x) / \theta\left(t_{0}\right)}\left|\phi_{2}(x)\right|^{2} \mathrm{~d} x \leq C \int_{\mathbb{R}^{n}} \mathrm{e}^{2 A(x) / \theta\left(t_{0}\right)}\left|\left\langle\zeta_{0}, \phi_{2}^{\prime}(x)\right\rangle\right|^{2} \mathrm{~d} x,
$$

which allows one to conclude as in the proof of Proposition 3.9.

\section{A.10. Proof of Theorem 7.6}

This proof is based on a proof provided in [36]. Its counterpart in the elliptic case can be found in [39]. In a sufficiently small neighborhood $V$ we place ourselves in normal geodesic coordinates. In such a coordinate system, the principal part of the Laplace operator $A$ takes the form

$$
A_{2}=-\partial_{x_{n}}^{2}-r\left(x, \partial_{x^{\prime}}\right), \quad x=\left(x^{\prime}, x_{n}\right)
$$

with $r\left(x, \xi^{\prime}\right)$ a homogeneous second-order polynomial in $\xi^{\prime}$ that satisfies

$$
r\left(x, \xi^{\prime}\right) \in \mathbb{R}, \quad \text { and } \quad C_{1}\left|\xi^{\prime}\right|^{2} \leq r\left(x, \xi^{\prime}\right) \leq C_{2}\left|\xi^{\prime}\right|^{2}, \quad x \in V, \xi^{\prime} \in \mathbb{R}^{n-1},
$$

for some $0<C_{1} \leq C_{2}<\infty$. The local coordinates are chosen such that $x_{0}$ is at the origin.

We set $\eta(t)=T^{2}(t(T-t))^{-1}$. Then $h=\varepsilon \theta=\varepsilon T^{2} \eta^{-1}$. We set $\hbar=\varepsilon T^{2}$ and $\hbar^{\prime}=\varepsilon T$. We shall impose $\hbar$ and $\hbar^{\prime}$ small in agreement with the statement of Theorem 7.6. To work on $\mathbb{R}$ for the time variable, instead of the finite interval $(0, T)$, we make the following change of variable:

$$
s(t)=\tan \left(\frac{\pi t}{T}-\frac{\pi}{2}\right)
$$

We note that $\partial_{t}=\frac{\mathfrak{a}(s)}{T} \partial_{s}$, with $\mathfrak{a}(s)=\pi\langle s\rangle^{2}$ with $\langle s\rangle=\left(1+s^{2}\right)^{\frac{1}{2}}$. The parabolic operator we consider becomes $P=\frac{\mathfrak{a}(s)}{T} \partial_{s}+A$ and we find

$$
\eta(s)=\pi^{2}\left(\frac{\pi}{2}+\arctan (s)\right)^{-1}\left(\frac{\pi}{2}-\arctan (s)\right)^{-1}, \quad h^{-1}=\eta(s) / \hbar .
$$

(We keep the notations $P, A, \eta$, in an abusive way.) In particular we have

$$
C\langle s\rangle \leq \eta(s) \leq C^{\prime}\langle s\rangle, \quad s \in \mathbb{R}, \quad \text { and } \quad C\langle s\rangle^{1-k} \leq\left|\eta^{(k)}(s)\right| \leq C^{\prime}\langle s\rangle^{1-k}, \quad k \in \mathbb{N} .
$$

We define tangential semi-classical $\psi$ DOs adapted to the parabolic problem we consider here. We denote by $S_{\mathcal{T}}^{m}$, the space of smooth functions $a\left(z, \zeta^{\prime}, h\right),\left(z, \zeta^{\prime}\right), z \in \mathbb{R}_{+}^{n+1}, \zeta^{\prime} \in \mathbb{R}^{n}$, defined for $\hbar \in\left(0, \hbar_{0}\right]$ for some $\hbar_{0}>0$, that satisfy the following property:

$$
\forall \alpha, \beta, \quad\left|\partial_{z}^{\alpha} \partial_{\zeta^{\prime}}^{\beta} a\left(z, \zeta^{\prime}, \hbar\right)\right| \leq C_{\alpha, \beta}\left\langle\zeta^{\prime}\right\rangle^{m-|\beta|}, \quad z \in \mathbb{R}_{+}^{n+1}, \zeta^{\prime} \in \mathbb{R}^{n}, \hbar \in\left(0, h_{0}\right) .
$$

Asymptotic series of such symbols as those defined in Section 2 can be considered. The notion of principal symbol is introduced similarly. The tangential $\psi$ DOs we shall consider are defined in the case $z=\left(s, x^{\prime}, x_{n}\right) \in \mathbb{R}^{n+1}$

\footnotetext{
${ }^{4}$ In fact, we can choose $\phi$ in the form $\phi(t, x)=\eta^{-\frac{1}{2}} \phi_{1}\left(\left(t-t_{0}\right) / \eta\right) \phi_{2}(x)$ with $\phi_{1} \in \mathscr{C}_{c}^{\infty}(\mathbb{R}), \int\left|\phi_{1}\right|^{2}=1$, and and $\eta>0$ sufficiently small. We then let $\eta$ go to 0 .
} 
and $\zeta^{\prime}=\left(\tau, \xi^{\prime}\right)$, with $s, \tau \in \mathbb{R}, x^{\prime}, \xi^{\prime} \in \mathbb{R}^{n-1}$ and $x_{n} \in \mathbb{R}^{+}$. We define $\langle s\rangle^{l} \Psi_{\mathcal{T}}^{m}$ as the space of tangential $\psi$ DOs $A=\operatorname{Op}(a)$, for $a \in\langle s\rangle^{l} S_{\mathcal{T}}^{m}$, formally defined by

$$
A u(s, x)=(2 \pi)^{-n} \hbar^{-n}\left(\hbar^{\prime}\right)^{-1} \iiint \int \mathrm{e}^{\mathrm{i}(s-t) \tau /\left(\hbar \hbar^{\prime}\right)+\mathrm{i}\left\langle x^{\prime}-y^{\prime}, \xi^{\prime}\right\rangle / \hbar} a\left(s, x, \tau, \xi^{\prime}, \hbar\right) u\left(t, y^{\prime}, x_{n}\right) \mathrm{d} t \mathrm{~d} y^{\prime} \mathrm{d} \tau \mathrm{d} \xi^{\prime} .
$$

If we let them act on a function $u$ that does not depend on $x_{n}$, they can be considered as regular $\psi$ DOs if we only consider the restriction of $A u$ on $x_{n}=0$. We shall also denote the principal symbol by $\sigma(A)$. We have the following quantizations:

$$
\sigma\left(\frac{\hbar \hbar^{\prime} \partial_{s}}{i}\right)=\tau, \quad \sigma\left(\frac{\hbar \partial_{x_{j}}}{i}\right)=\xi_{i}
$$

We set $D_{s}=\frac{\hbar \hbar^{\prime} \partial_{s}}{i}$ and $D_{x_{j}}=\frac{\hbar \partial_{x_{j}}}{i}$.

If we set $M=\left\langle\left(\tau, \xi^{\prime}\right)\right\rangle \in S_{\mathcal{T}}^{1}$ we have the following regularity result: if $a \in\langle s\rangle^{l} S_{\mathcal{T}}^{m}, l, m \in \mathbb{R}$, then there exists $C>0$ such that

$$
\|\mathrm{Op}(a) u\| \leq C\left\|\langle s\rangle^{l} \mathrm{Op}\left(M^{m}\right) u\right\| .
$$

The composition formula for tangential symbols, $a \in\langle s\rangle^{l} S_{\mathcal{T}}^{m}, b \in\langle s\rangle^{l^{\prime}} S_{\mathcal{T}}^{m^{\prime}}$, is given by

$$
(a \sharp b)\left(s, x, \tau, \xi^{\prime}, \hbar\right) \sim \sum_{|\alpha|} \hbar^{|\alpha|}\left(\hbar^{\prime}\right)^{\alpha_{1}} \frac{(-i)^{|\alpha|}}{\alpha !}\left(\partial_{\tau}^{\alpha_{1}} \partial_{\xi^{\prime}}^{\alpha_{2}} a\right)\left(\partial_{s}^{\alpha_{1}} \partial_{x^{\prime}}^{\alpha_{2}} b\right)\left(s, x, \tau, \xi^{\prime}, \hbar\right),
$$

with $\alpha=\left(\alpha_{1}, \alpha_{2}\right), \alpha_{1} \in \mathbb{N}, \alpha_{2} \in \mathbb{N}^{n-1}$, and yields a tangential symbol in $\langle s\rangle^{l+l^{\prime}} S_{\mathcal{T}}^{m+m^{\prime}}$.

We now make the following change of variables in the $x$ direction.

$$
y=\mathfrak{b}(s) x, \quad \text { with } \mathfrak{b}(s)=\mathfrak{a}(s)^{\frac{1}{2}} .
$$

If we set $\mathfrak{P}=P / \mathfrak{a}(s)$, its principal part is given by

$$
\mathfrak{P}_{2}=\frac{1}{T} \partial_{s}-\partial_{y_{n}}^{2}-\mathfrak{r}\left(y, \partial_{y^{\prime}}\right), \quad \mathfrak{r}\left(y, \partial_{y^{\prime}}\right)=r\left(y / \mathfrak{b}(s), \partial_{y^{\prime}}\right) .
$$

We shall prove a Carleman estimate for this operator before moving back to the original coordinates. In the sequel it is important to remember that $x=y / \mathfrak{b}(s)$ remains in the compact domain $K$.

We set

$$
\phi(s, y)=\varphi(y / \mathfrak{b}(s)), \quad \phi_{x}(s, y)=\frac{\eta(s)}{\mathfrak{b}(s)} \partial_{x} \varphi(y / \mathfrak{b}(s)) .
$$

Note that $\phi_{x}$ does not stand for $\partial_{x} \phi$ but is in fact equal to $\eta \partial_{x} \phi$. This notation will however be convenient below since the calculus we have introduced uses $\hbar$ and $\hbar^{\prime}$ for small parameters instead of $h=\hbar \eta^{-1}$.

Remark A.6. With the definitions of $\mathfrak{r}$ and $\phi$, we find that derivatives of the symbols with respect to $s$ and $y$ generate a gain of a factor $\langle s\rangle^{-1}$. This will be taken into account in the application of the composition formula (A.13).

Introduce $v=\mathrm{e}^{\phi / h} u=\mathrm{e}^{\phi \eta / \hbar} u$ and $\mathfrak{P}_{\varphi}=\hbar^{2} \mathrm{e}^{\phi / h} \mathfrak{P e}^{-\phi / h}$. We have $\mathfrak{P}_{\varphi} v=\hbar^{2} \mathrm{e}^{\phi / h} \mathfrak{P} u=g$. We define the following symmetric operators $\tilde{Q}_{2}=\left(\mathfrak{P}_{\varphi}+\mathfrak{P}_{\varphi}^{*}\right) / 2, \tilde{Q}_{1}=\left(\mathfrak{P}_{\varphi}-\mathfrak{P}_{\varphi}^{*}\right) /(2 i)$, with

$$
\tilde{Q}_{2}=D_{y_{n}}^{2}+Q_{2}, \quad \tilde{Q}_{1}=\left(D_{y_{n}} \phi_{x_{n}}+\phi_{x_{n}} D_{y_{n}}\right)+2 Q_{1}
$$


with respective principal symbols

$$
\begin{aligned}
& \tilde{q}_{2}=\xi_{n}^{2}+q_{2}, \quad q_{2}=\mathfrak{r}\left(y, \xi^{\prime}\right)-\mathfrak{r}\left(y, \phi_{x^{\prime}}\right)-\phi_{x_{n}}^{2} \in S_{\mathcal{T}}^{2}, \\
& \tilde{q}_{1}=2 \xi_{n} \phi_{x_{n}}+2 q_{1}, \quad q_{1}=\frac{\tau}{2}+\tilde{\mathfrak{r}}\left(y, \xi^{\prime}, \phi_{x^{\prime}}\right) \in S_{\mathcal{T}}^{1}
\end{aligned}
$$

where $\tilde{\mathfrak{r}}(y, .,$.$) is the symmetric bilinear form associated with the real quadratic form \mathfrak{r}\left(x, \xi^{\prime}\right)$.

We note that

$$
\begin{aligned}
& \left(w_{1}, \tilde{Q}_{2} w_{2}\right)=\left(\tilde{Q}_{2} w_{1}, w_{2}\right)-i \hbar\left[\left(\left.w_{1}\right|_{y_{n}=0^{+}},\left.D_{y_{n}} w_{2}\right|_{y_{n}=0^{+}}\right)_{0}+\left(\left.D_{y_{n}} w_{1}\right|_{y_{n}=0^{+}},\left.w_{2}\right|_{y_{n}=0^{+}}\right)_{0}\right], \\
& \left(w_{1}, \tilde{Q}_{1} w_{2}\right)=\left(\tilde{Q}_{1} w_{1}, w_{2}\right)-2 i \hbar\left(\left.\phi_{x_{n}} w_{1}\right|_{y_{n}=0^{+}},\left.w_{2}\right|_{y_{n}=0^{+}}\right)_{0},
\end{aligned}
$$

for $w_{1}$ and $w_{2}$ smooth, where $(., .)_{0}$ is the $L^{2}$ hermitian inner-product in $\left\{y_{n}=0\right\}$, and we thus obtain

$$
\|g\|^{2}=\left\|\tilde{Q}_{1} v\right\|^{2}+\left\|\tilde{Q}_{2} v\right\|^{2}+i\left(\left[\tilde{Q}_{2}, \tilde{Q}_{1}\right] v, v\right)+\hbar \mathscr{B}(v)
$$

with

$$
\mathscr{B}(v)=\left(\left.\tilde{Q}_{1} v\right|_{y_{n}=0^{+}},\left.D_{y_{n}} v\right|_{y_{n}=0^{+}}\right)_{0}+\left(\left.\left(D_{y_{n}} \tilde{Q}_{1}-2 \phi_{x_{n}} \tilde{Q}_{2}\right) v\right|_{y_{n}=0^{+}},\left.v\right|_{y_{n}=0^{+}}\right)_{0},
$$

which, as $\left.v\right|_{y_{n}=0^{+}}=0$, reduces to

$$
\mathscr{B}(v)=2\left(\left.\phi_{x_{n}} D_{y_{n}} v\right|_{y_{n}=0^{+}},\left.D_{y_{n}} v\right|_{y_{n}=0^{+}}\right)_{0} \geq 0, \quad \text { as } \phi_{x_{n}}>0
$$

We observe that we have $i\left[\tilde{Q}_{2}, \tilde{Q}_{1}\right]=\hbar\left(\tilde{H}_{0} D_{y_{n}}^{2}+\tilde{H}_{1} D_{y_{n}}+\tilde{H}_{2}\right)$, where $\tilde{H}_{j} \in\langle s\rangle^{-1} \Psi_{\mathcal{T}}^{j}, j=0,1,2$. We then note that $D_{y_{n}}^{2}-\tilde{Q}_{2} \in \Psi_{\mathcal{T}}^{2}$ and $D_{y_{n}}-\frac{1}{2 \phi_{x_{n}}} \tilde{Q}_{1} \in \Psi_{\mathcal{T}}^{1}$. We thus find

$$
i\left[\tilde{Q}_{2}, \tilde{Q}_{1}\right]=\hbar\left(H_{0} \tilde{Q}_{2}+H_{1} \tilde{Q}_{1}+H_{2}\right), \quad H_{j} \in\langle s\rangle^{-1} \Psi_{\mathcal{T}}^{j}, j=0,1,2
$$

We have the following lemma, which proof is given below.

Lemma A.7. For $\mu$ sufficiently large and $\hbar$ and $\hbar^{\prime}$ sufficiently small, there exists $C>0$ such that

$$
\langle s\rangle^{-1} S_{\mathcal{T}}^{2} \ni \mu \frac{\left(q_{1}^{2}+\phi_{x_{n}}^{2} q_{2}\right)^{2}}{\langle s\rangle M^{2}}+\sigma\left(H_{2}\right) \geq C\langle s\rangle^{-1} M^{2} .
$$

Applying the Gårding inequality in the tangential directions (including the time direction) we thus obtain, for $\hbar$ sufficiently small,

$$
\begin{aligned}
\|g\|^{2} \geq\left\|\tilde{Q}_{1} v\right\|^{2}+\left\|\tilde{Q}_{2} v\right\|^{2}+\hbar \operatorname{Re}\left(H_{0} \tilde{Q}_{2} v, v\right) & +\hbar \operatorname{Re}\left(H_{1} \tilde{Q}_{1} v, v\right) \\
& +C \hbar\left\|\langle s\rangle^{-\frac{1}{2}} \mathrm{Op}(M) v\right\|^{2}-\hbar \operatorname{Re}\left(\left(Q_{1}^{2}+\phi_{x_{n}}^{2} Q_{2}\right) v, G\langle s\rangle^{-1} v\right)
\end{aligned}
$$

where $G \in \Psi_{\mathcal{T}}^{0}$ and $\sigma(G)=\mu \frac{q_{1}^{2}+\phi_{x_{n}}^{2} q_{2}}{M^{2}} \in S_{\mathcal{T}}^{0}$.

We first see that we have

$$
\begin{aligned}
& \hbar\left|\left(H_{0} \tilde{Q}_{2} v, v\right)\right| \leq \hbar\left\|\tilde{Q}_{2} v\right\|\left\|\langle s\rangle^{-1} v\right\| \leq C \hbar^{\frac{1}{2}}\left\|\tilde{Q}_{2} v\right\|^{2}+C^{\prime} \hbar^{\frac{3}{2}}\left\|\langle s\rangle^{-1} v\right\|^{2}, \\
& \hbar\left|\left(H_{1} \tilde{Q}_{1} v, v\right)\right| \leq \hbar\left\|\tilde{Q}_{1} v\right\|\left\|\langle s\rangle^{-1} \mathrm{Op}(M) v\right\| \leq C \hbar^{\frac{1}{2}}\left\|\tilde{Q}_{1} v\right\|^{2}+C^{\prime} \hbar^{\frac{3}{2}}\left\|\langle s\rangle^{-1} \mathrm{Op}(M) v\right\|^{2} .
\end{aligned}
$$

From the form of $\tilde{Q}_{1}$ we deduce the following lemma. 
Lemma A.8. We have $\left\|\langle s\rangle^{-\frac{1}{2}} D_{y_{n}} v\right\| \leq C\left\|\tilde{Q}_{1} v\right\|+C\left\|\langle s\rangle^{-\frac{1}{2}} \mathrm{Op}(M) v\right\|$.

Next since

$$
Q_{1}=\frac{1}{2}\left(\tilde{Q}_{1}-\left[D_{y_{n}}, \phi_{x_{n}}\right]\right)-\phi_{x_{n}} D_{y_{n}}
$$

we compute $Q_{1}^{2}+\phi_{x_{n}}^{2} Q_{2}=-Q_{1} \phi_{x_{n}} D_{y_{n}}+\frac{Q_{1}}{2}\left(\tilde{Q}_{1}-\left[D_{y_{n}}, \phi_{x_{n}}\right]\right)+\phi_{x_{n}}^{2}\left(\tilde{Q}_{2}-D_{y_{n}}^{2}\right)$. Using (A.19) a second time we have

$$
Q_{1}^{2}+\phi_{x_{n}}^{2} Q_{2}=\left(\phi_{x_{n}} D_{y_{n}}-\frac{1}{2}\left(\tilde{Q}_{1}-\left[D_{y_{n}}, \phi_{x_{n}}\right]\right)\right) \phi_{x_{n}} D_{y_{n}}+\frac{Q_{1}}{2}\left(\tilde{Q}_{1}-\left[D_{y_{n}}, \phi_{x_{n}}\right]\right)+\phi_{x_{n}}^{2}\left(\tilde{Q}_{2}-D_{y_{n}}^{2}\right),
$$

which reads

$$
Q_{1}^{2}+\phi_{x_{n}}^{2} Q_{2} \in \phi_{x_{n}}^{2} \tilde{Q}_{2}-\frac{1}{2} D_{y_{n}} \phi_{x_{n}} \tilde{Q}_{1}+\Psi_{\mathcal{T}}^{1} \tilde{Q}_{1}+\hbar\langle s\rangle^{-1} \Psi_{\mathcal{T}}^{0} D_{y_{n}}+\hbar\langle s\rangle^{-1} \Psi_{\mathcal{T}}^{1}
$$

We note that

$$
\hbar\left|\left(\phi_{x_{n}}^{2} \tilde{Q}_{2} v, G\langle s\rangle^{-1} v\right)\right| \leq \hbar^{\frac{1}{2}} C\left\|\tilde{Q}_{2} v\right\|^{2}+\hbar^{\frac{3}{2}} C\left\|\langle s\rangle^{-1} v\right\|^{2}
$$

and

$$
\hbar \operatorname{Re}\left(\frac{1}{2} D_{y_{n}} \phi_{x_{n}} \tilde{Q}_{1} v, G\langle s\rangle^{-1} v\right)=\frac{\hbar}{2} \operatorname{Re}\left(\phi_{x_{n}} \tilde{Q}_{1} v, D_{y_{n}} G\langle s\rangle^{-1} v\right)-\hbar^{2} \operatorname{Re}\left(\left.\frac{\phi_{x_{n}}}{2 i} \tilde{Q}_{1} v\right|_{y_{n}=0^{+}},\left.G\langle s\rangle^{-1} v\right|_{y_{n}=0^{+}}\right)_{0},
$$

by integration by parts. The last term vanishes as $\left.v\right|_{y_{n}=0^{+}}=0$. We thus obtain

$$
\begin{aligned}
& \left|\hbar \operatorname{Re}\left(\left(Q_{1}^{2}+\phi_{x_{n}}^{2} Q_{2}\right) v, G\langle s\rangle^{-1} v\right)\right| \\
& \quad \leq C\left(\hbar^{\frac{1}{2}}\left\|\tilde{Q}_{1} v\right\|^{2}+\hbar^{\frac{1}{2}}\left\|\tilde{Q}_{2} v\right\|^{2}+\hbar^{\frac{3}{2}}\left\|\langle s\rangle^{-1} D_{y_{n}} v\right\|^{2}+\hbar^{\frac{3}{2}}\left\|\langle s\rangle^{-1} v\right\|^{2}+\hbar^{\frac{3}{2}}\left\|\langle s\rangle^{-1} \mathrm{Op}(M) v\right\|^{2}\right) .
\end{aligned}
$$

By choosing $\hbar$ sufficiently small, from (A.16), (A.17), (A.18) and (A.21), and Lemma A.8, we obtain

$$
\left\|\mathfrak{P}_{\varphi} v\right\|^{2} \geq C \hbar\left(\left\|\langle s\rangle^{-\frac{1}{2}} \mathrm{Op}(M) v\right\|^{2}+\left\|\langle s\rangle^{-\frac{1}{2}} D_{y_{n}} v\right\|^{2}\right) \geq C \hbar\left(\left\|\langle s\rangle^{-\frac{1}{2}} v\right\|^{2}+\hbar^{2}\left\|\langle s\rangle^{-\frac{1}{2}} \nabla_{y} v\right\|^{2}\right) .
$$

Arguing as in the proof of Theorem 3.5 we obtain

$$
\hbar^{4}\left\|\mathrm{e}^{\phi / h} \mathfrak{P} u\right\|^{2} \geq C \hbar\left(\left\|\mathrm{e}^{\phi / h}\langle s\rangle^{-\frac{1}{2}} u\right\|^{2}+\hbar^{2}\left\|\mathrm{e}^{\phi / h}\langle s\rangle^{-\frac{1}{2}} \nabla_{y} u\right\|^{2}\right) .
$$

Moving back to the $x$ coordinate we find

$$
\hbar^{4}\left\|\mathrm{e}^{\varphi / h}\langle s\rangle^{-\frac{n}{2}-2} P u\right\|^{2} \geq C \hbar\left(\left\|\mathrm{e}^{\varphi / h}\langle s\rangle^{-\frac{n+1}{2}} u\right\|^{2}+\hbar^{2}\left\|\mathrm{e}^{\varphi / h}\langle s\rangle^{-\frac{n+3}{2}} \nabla_{x} u\right\|^{2}\right) .
$$

We now proceed with writing the local Carleman estimate we have obtained with the original time variable $t \in(0, T)$. From (A.10) we have $\mathrm{d} s=\frac{\mathfrak{a}(s(t))}{T} \mathrm{~d} t=\frac{\pi}{T}\langle s(t)\rangle^{2} \mathrm{~d} t$. We then have

$$
\hbar^{4}\left\|\mathrm{e}^{\varphi / h} \eta(t)^{-\frac{n}{2}-1} P u\right\|^{2} \geq C \hbar\left(\left\|\mathrm{e}^{\varphi / h} \eta(t)^{-\frac{n-1}{2}} u\right\|^{2}+\hbar^{2}\left\|\mathrm{e}^{\varphi / h} \eta(t)^{-\frac{n+1}{2}} \nabla_{x} u\right\|^{2}\right),
$$


as $\eta(t) / C \leq\langle s(t)\rangle \leq C \eta(t)$. If we now change $u$ into $\eta(t)^{\frac{n}{2}-1} u$, which is possible because of the fast decay of $\mathrm{e}^{\varphi / h}$ as $t \rightarrow 0$ or $T$, we achieve

$$
\hbar^{4}\left\|\mathrm{e}^{\varphi / h} \eta(t)^{-2} P u\right\|^{2} \geq C \hbar\left(\left\|\mathrm{e}^{\varphi / h} \eta(t)^{-\frac{1}{2}} u\right\|^{2}+\hbar^{2}\left\|\mathrm{e}^{\varphi / h} \eta(t)^{-\frac{3}{2}} \nabla_{x} u\right\|^{2}\right) .
$$

In fact, we chose $\varepsilon T=\hbar^{\prime}$ sufficiently small to absorb the commutator $\left[\partial_{t}, \eta(t)^{\frac{n}{2}-1}\right]$ generated by this change of unknown function. Recalling that $\hbar / \eta=h$ this concludes the proof.

Proof of Lemma A.7 We denote by $\{., .\}_{s}$, and $\{., .\}_{x}$ the Poisson brackets with respect to the $s, \tau$ variables on the one hand and the $x, \xi$ variables on the other hand. We have

$$
\langle s\rangle^{-1} S^{2} \ni \sigma\left(\frac{i}{\hbar}\left[\tilde{Q}_{2}, \tilde{Q}_{1}\right]\right)=\underbrace{\hbar^{\prime}\left\{\tilde{q}_{2}, \tilde{q}_{1}\right\}_{s}}_{\in h^{\prime}\langle s\rangle^{-1} S^{1}}+\left\{\tilde{a}_{2}, \tilde{a}_{1}\right\}_{x} .
$$

For the property we aim to prove we may thus focus on the second term that belongs to $\langle s\rangle^{-1} S^{2}$. We introduce the map

$$
\kappa:(s, y, \tau, \xi) \mapsto\left(s, \frac{y}{\mathfrak{b}(s)},\left(\frac{\mathfrak{b}}{\eta}(s)\right)^{2} \tau,\left(\frac{\eta}{\mathfrak{b}(s)}\right)^{-1} \xi\right),
$$

and set $\tilde{q}_{j} \circ \kappa^{-1}=\left(\frac{\eta}{b}(s)\right)^{2} \tilde{a}_{j}, j=1,2$. We find

$$
\begin{aligned}
& \tilde{a}_{2}(s, x, \tau, \xi)=\xi_{n}^{2}+a_{2} \in S^{2}, \quad a_{2}=r\left(x, \xi^{\prime}\right)-r\left(x, \partial_{x^{\prime}} \varphi\right)-\left(\partial_{x_{n}} \varphi\right)^{2} \in S_{\mathcal{T}}^{2}, \\
& \tilde{a}_{1}(s, x, \tau, \xi)=2 \xi_{n}\left(\partial_{x_{n}} \varphi\right)+2 a_{1} \in S^{1}, \quad a_{1}=\frac{\tau}{2}+\tilde{r}\left(x, \xi^{\prime}, \partial_{x^{\prime}} \varphi\right) \in S_{\mathcal{T}}^{1},
\end{aligned}
$$

where $\tilde{r}(y, .,$.$) is the symmetric bilinear form associated with the real quadratic form r\left(x, \xi^{\prime}\right)$. Recall that the variable $x$ remains in the compact set $K$, as opposed to $y=\mathfrak{b}(s) x$. We observe that $\left\{\tilde{q}_{2}, \tilde{q}_{1}\right\}_{x}=\frac{\eta^{3}}{\mathfrak{b}^{4}}\left\{\tilde{a}_{2}, \tilde{a}_{1}\right\}_{x} \circ \kappa$. In particular we have

$$
\left\{\tilde{q}_{2}, \tilde{q}_{1}\right\}_{x}=h_{0} \tilde{q}_{2}+h_{1} \tilde{q}_{1}+h_{2}, \quad\left\{\tilde{a}_{2}, \tilde{a}_{1}\right\}_{x}=\ell_{0} \tilde{a}_{2}+\ell_{1} \tilde{a}_{1}+\ell_{2},
$$

with $h_{j}=\sigma\left(H_{j}\right) \in\langle s\rangle^{-1} S_{\mathcal{T}}^{j}$ and $\ell_{j} \in S_{\mathcal{T}}^{j}$. We find $h_{2}=\frac{\eta^{3}}{\mathfrak{b}^{4}} \ell_{2} \circ \kappa$. We set $M^{\prime}=M \circ \kappa^{-1}$. We note that $M^{\prime} \sim M$. We set

$$
\nu=\mu \frac{\left(a_{1}^{2}+\left(\partial_{x_{n}} \varphi\right)^{2} a_{2}\right)^{2}}{M^{\prime 2}}+\frac{\langle s\rangle}{\eta} \ell_{2} \in S_{\mathcal{T}}^{2} .
$$

We now prove that $\nu \geq C M^{2}$ for $\mu$ sufficiently large. For $\left|\left(\tau, \xi^{\prime}\right)\right|$ large, say $\left|\left(\tau, \xi^{\prime}\right)\right| \geq R$, we clearly have $\nu \geq C M^{2}$. We may thus restrict ourselves to the case of the variables $(x, \tau, \xi)$ in a compact set. Recall that $s=s(t)$ and observe that $\nu$ is smooth with respect to $t \in[0, T]$. Hence all the variables $(t, x, \tau, \xi)$ lay in a compact set. We consider the case $\hbar=\hbar^{\prime}=0$. Assume that $a_{1}^{2}+\left(\partial_{x_{n}} \varphi\right)^{2} a_{2}$ and chose $\xi_{n}=-\left(a_{1} / \partial_{x_{n}} \varphi\right)$. Then $\tilde{a}_{1}=\tilde{a}_{2}=0$. By the sub-elliptic property (7.1) (which is invariant by change of variables) we obtain $\left\{\tilde{a}_{2}, \tilde{a}_{1}\right\}_{x}=l_{2}>0$. It follows that for $\mu$ sufficiently large $\nu / M^{2} \geq C>0$ in this case as well. Relaxing the condition $\hbar=\hbar^{\prime}=0$ only affects the constant $C$ for $\hbar, \hbar^{\prime}$ positive and sufficiently small.

We conclude the proof since we have

$$
h_{2}+\mu \frac{\left(q_{1}^{2}+\phi_{x_{n}}^{2} q_{2}\right)^{2}}{\langle s\rangle M^{2}}=\langle s\rangle^{-1}\left(\frac{\eta}{\mathfrak{b}}\right)^{4} \nu \circ \kappa .
$$




\section{REFERENCES}

[1] S. Agmon, Lectures on Elliptic Boundary Values Problems. Van Nostrand (1965).

[2] S. Alinhac and P. Gérard, Opérateurs Pseudo-Différentiels et Théorème de Nash-Moser. Éditions du CNRS (1991).

[3] J.-P. Aubin and I. Ekeland, Applied Non Linear Analysis. John Wiley \& Sons, New York (1984).

[4] V. Barbu, Exact controllability of the superlinear heat equation. Appl. Math. Optim. 42 (2000) 73-89.

[5] M. Bellassoued, Carleman estimates and distribution of resonances for the transparent obstacle and application to the stabilization. Asymptotic Anal. 35 (2003) 257-279.

[6] A. Benabdallah and M.G. Naso, Null controllability of a thermoelastic plate. Abstr. Appl. Anal. 7 (2002) 585-599.

[7] A. Benabdallah, Y. Dermenjian and J. Le Rousseau, Carleman estimates for the one-dimensional heat equation with a discontinuous coefficient and applications to controllability and an inverse problem. J. Math. Anal. Appl. 336 (2007) 865887.

[8] A. Benabdallah, Y. Dermenjian and J. Le Rousseau, On the controllability of linear parabolic equations with an arbitrary control location for stratified media. C. R. Acad. Sci. Paris, Ser. I 344 (2007) 357-362.

[9] M. Boulakia and A. Osses, Local null controllability of a two-dimensional fluid-structure interaction problem. ESAIM Control Optim. Calc. Var. 14 (2008) 1-42.

[10] H. Brezis, Analyse Fonctionnelle. Masson, Paris (1983).

[11] T. Carleman, Sur un problème d'unicité pour les systèmes d'équations aux dérivées partielles à deux variables indépendantes. Ark. Mat. Astr. Fys. 26B (1939) 1-9.

[12] L. de Teresa, Insensitizing controls for a semilinear heat equation. Comm. Partial Differential Equations 25 (2000) 39-72.

[13] M. Dimassi and J. Sjöstrand, Spectral Asymptotics in the Semi-classical Limit, London Mathematical Society Lecture Note Series 268. Cambridge University Press, Cambridge (1999).

[14] A. Doubova, E. Fernandez-Cara, M. Gonzales-Burgos and E. Zuazua, On the controllability of parabolic systems with a nonlinear term involving the state and the gradient. SIAM J. Control Optim. 41 (2002) 798-819.

[15] A. Doubova, A. Osses and J.-P. Puel, Exact controllability to trajectories for semilinear heat equations with discontinuous diffusion coefficients. ESAIM: COCV 8 (2002) 621-661.

[16] E. Fernández-Cara and S. Guerrero, Global Carleman inequalities for parabolic systems and application to controllability. SIAM J. Control Optim. 45 (2006) 1395-1446.

[17] E. Fernández-Cara and E. Zuazua, The cost of approximate controllability for heat equations: the linear case. Adv. Differential Equations 5 (2000) 465-514.

[18] E. Fernández-Cara and E. Zuazua, Null and approximate controllability for weakly blowing up semilinear heat equations. Ann. Inst. Henri Poincaré, Analyse non linéaire 17 (2000) 583-616.

[19] E. Fernández-Cara, S. Guerrero, O.Yu. Imanuvilov and J.-P. Puel, Local exact controllability of the Navier-Stokes system. J. Math. Pures Appl. 83 (2004) 1501-1542.

[20] E. Fernández-Cara, S. Guerrero, O.Yu. Imanuvilov and J.-P. Puel, Some controllability results for the $N$-dimensional NavierStokes and Boussinesq systems with $N-1$ scalar controls. SIAM J. Control Optim. 45 (2006) 146-173.

[21] C. Fabre and G. Lebeau, Prolongement unique des solutions de l'equation de Stokes. Comm. Partial Differential Equations 21 (1996) 573-596.

[22] A. Fursikov and O.Yu. Imanuvilov, Controllability of evolution equations, Lecture Notes 34. Seoul National University, Korea (1996).

[23] M. González-Burgos and R. Pérez-García, Controllability results for some nonlinear coupled parabolic systems by one control force. Asymptotic Anal. 46 (2006) 123-162.

[24] A. Grigis and J. Sjöstrand, Microlocal Analysis for Differential Operators. Cambridge University Press, Cambridge (1994).

[25] L. Hörmander, Linear Partial Differential Operators. Springer-Verlag, Berlin (1963).

[26] L. Hörmander, The Analysis of Linear Partial Differential Operators IV. Springer-Verlag (1985).

[27] L. Hörmander, The Analysis of Linear Partial Differential Operators III. Springer-Verlag (1985). 2nd printing 1994.

[28] L. Hörmander, The Analysis of Linear Partial Differential Operators I. 2nd edition, Springer-Verlag (1990).

[29] O.Yu. Imanuvilov, Remarks on the exact controllability of Navier-Stokes equations. ESAIM: COCV 6 (2001) 39-72.

[30] O.Yu. Imanuvilov and T. Takahashi, Exact controllability of a fluid-rigid body system. J. Math. Pures Appl. 87 (2007) 408-437.

[31] D. Jerison and G. Lebeau, Harmonic analysis and partial differential equations (Chicago, IL, 1996). chapter Nodal sets of sums of eigenfunctions, Chicago Lectures in Mathematics, The University of Chicago Press, Chicago (1999) 223-239.

[32] F. Ammar Khodja, A. Benabdallah and C. Dupaix, Null controllability of some reaction-diffusion systems with one control force. J. Math. Anal. Appl. 320 (2006) 928-943.

[33] F. Ammar Khodja, A. Benabdallah, C. Dupaix and I. Kostin, Null-controllability of some systems of parabolic type by one control force. ESAIM: COCV 11 (2005) 426-448.

[34] J. Le Rousseau, Carleman estimates and controllability results for the one-dimensional heat equation with $B V$ coefficients. J. Differential Equations 233 (2007) 417-447. 
[35] J. Le Rousseau and L. Robbiano, Carleman estimate for elliptic operators with coefficents with jumps at an interface in arbitrary dimension and application to the null controllability of linear parabolic equations. Arch. Rational Mech. Anal. 105 (2010) 953-990.

[36] J. Le Rousseau and L. Robbiano, Local and global Carleman estimates for parabolic operators with coefficients with jumps at interfaces. Invent. Math. 183 (2011) 245-336.

[37] M. Léautaud, Spectral inequalities for non-selfadjoint elliptic operators and application to the null-controllability of parabolic systems. J. Funct. Anal. 258 (2010) 2739-2778.

[38] G. Lebeau, Cours sur les inégalités de Carleman, Mastère Equations aux Dérivées Partielles et Applications. Faculté des Sciences de Tunis, Tunisie (2005).

[39] G. Lebeau and L. Robbiano, Contrôle exact de l'équation de la chaleur. Comm. Partial Differential Equations 20 (1995) $335-356$.

[40] G. Lebeau and L. Robbiano, Stabilisation de l'équation des ondes par le bord. Duke Math. J. 86 (1997) 465-491.

[41] G. Lebeau and E. Zuazua, Null-controllability of a system of linear thermoelasticity. Arch. Rational Mech. Anal. 141 (1998) $297-329$.

[42] A. Martinez, An Introduction to Semiclassical and Microlocal Analysis. Springer-Verlag (2002).

[43] S. Micu and E. Zuazua, On the lack of null-controllability of the heat equation on the half space. Port. Math. (N.S.) 58 (2001) 1-24.

[44] L. Miller, On the null-controllability of the heat equation in unbounded domains. Bull. Sci. Math. 129 (2005) 175-185.

[45] L. Miller, On the controllability of anomalous diffusions generated by the fractional laplacian. Mathematics of Control, Signals, and Systems 3 (2006) 260-271.

[46] L. Miller, Unique continuation estimates for sums of semiclassical eigenfunctions and null-controllability from cones. Preprint (2008). http://hal.archives-ouvertes.fr/hal-00411840/fr.

[47] L. Miller, A direct Lebeau-Robbiano strategy for the observability of heat-like semigroups. Discrete Contin. Dyn. Syst. Ser. B 14 (2010) 1465-1485.

[48] L. Robbiano, Théorème d'unicité adapté au contrôle des solutions des problèmes hyperboliques. Comm. Partial Differential Equations 16 (1991) 789-800.

[49] L. Robbiano, Fonction de coût et contrôle des solutions des équations hyperboliques. Asymptotic Anal. 10 (1995) $95-115$.

[50] D. Robert, Autour de l'Approximation Semi-Classique, Progress in Mathematics 68. Birkhäuser Boston, Boston, MA (1987).

[51] J.-C. Saut and B. Scheurer, Unique continuation for some evolution equations. J. Differential Equations 66 (1987) $118-139$.

[52] M.A. Shubin, Pseudodifferential Operators and Spectral Theory. 2nd edition, Springer-Verlag, Berlin Heidelberg (2001).

[53] D. Tataru, Carleman estimates and unique continuation for the Schroedinger equation. Differential Integral Equations 8 (1995) 901-905.

[54] D. Tataru, Unique continuation for solutions to PDE's; between Hörmander's theorem and Holmgren's theorem. Comm. Partial Differential Equations 20 (1995) 855-884.

[55] M.E. Taylor, Pseudodifferential Operators. Princeton University Press, Princeton, New Jersey (1981).

[56] M.E. Taylor, Partial Differential Equations 2: Qualitative Studies of Linear Equations, Applied Mathematical Sciences 116. Springer-Verlag, New-York (1996).

[57] G. Tenenbaum and M. Tucsnak, On the null-controllability of diffusion equations. preprint (2009).

[58] F. Trèves, Topological Vector Spaces, Distributions and Kernels. Academic Press, New York (1967).

[59] C. Zuily, Uniqueness and Non Uniqueness in the Cauchy Problem. Birkhäuser, Progress in mathematics (1983). 